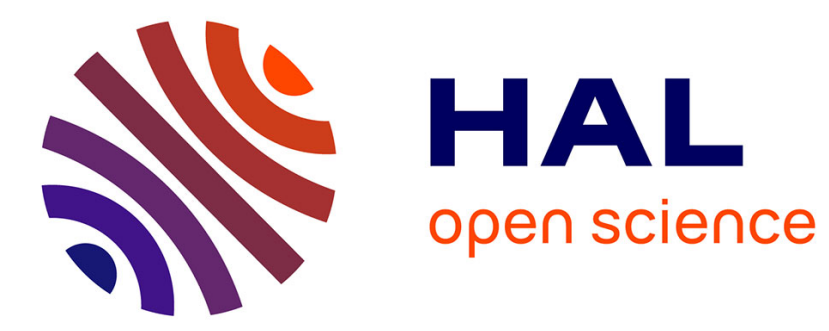

\title{
Control in dormancy or eradication of cancer stem cells: Mathematical modeling and stability issues
}

Walid Djema, Catherine Bonnet, Frédéric Mazenc, Jean Clairambault, Emilia Fridman, Pierre Hirsch, François Delhommeau

\section{- To cite this version:}

Walid Djema, Catherine Bonnet, Frédéric Mazenc, Jean Clairambault, Emilia Fridman, et al.. Control in dormancy or eradication of cancer stem cells: Mathematical modeling and stability issues. Journal of Theoretical Biology, 2018, 449, pp.103 - 123. 10.1016/j.jtbi.2018.03.038 . hal-01852154

\author{
HAL Id: hal-01852154 \\ https://hal.inria.fr/hal-01852154
}

Submitted on 31 Jul 2018

HAL is a multi-disciplinary open access archive for the deposit and dissemination of scientific research documents, whether they are published or not. The documents may come from teaching and research institutions in France or abroad, or from public or private research centers.
L'archive ouverte pluridisciplinaire HAL, est destinée au dépôt et à la diffusion de documents scientifiques de niveau recherche, publiés ou non, émanant des établissements d'enseignement et de recherche français ou étrangers, des laboratoires publics ou privés. 
Journal of Theoretical Biology 449 (2018) 103-123

\title{
Control in Dormancy or Eradication of Cancer Stem Cells: Mathematical Modeling and Stability Issues ${ }^{\text {th }}$
}

\author{
Walid Djema ${ }^{1, *}$, Catherine Bonnet ${ }^{2}$, Frédéric Mazenc ${ }^{2}$, Jean Clairambault ${ }^{3}$, Emilia \\ Fridman ${ }^{4}$, Pierre Hirsch $^{5}$, François Delhommeau ${ }^{5}$
}

\begin{abstract}
Objective: Modeling and analysis of cell population dynamics enhance our understanding of cancer. Here we introduce and explore a new model that may apply to many tissues.

Analyses: An age-structured model describing coexistence between mutated and ordinary stem cells is developed and explored. The model is transformed into a nonlinear time-delay system governing the dynamics of healthy cells, coupled to a nonlinear differential-difference system describing dynamics of unhealthy cells. Its main features are highlighted and an advanced stability analysis of several steady states is performed, through specific Lyapunov-like functionals for descriptor-type systems.

Results: We propose a biologically based model endowed with rich dynamics. It incorporates a new parameter representing immunoediting processes, including the case where proliferation of cancer cells is locally kept under check by the immune cells. It also considers the overproliferation of cancer stem cells, modeled as a subpopulation of mutated cells that is constantly active in cell division. The analysis that we perform here reveals the conditions of existence of several steady states, including the case of cancer dormancy, in the coupled model of interest. Our study suggests that cancer dormancy may result from a plastic sensitivity of mutated cells to their shared environment, different from that - fixed - of healthy cells, and this is related to an action (or lack of action) of the immune system. Next, the stability analysis that we perform is essentially oriented towards the determination of sufficient conditions, depending on all the model parameters, that ensure either a regionally (i.e., locally) stable dormancy steady state or eradication of unhealthy cells. Finally, we discuss some biological interpretations, with regards to our findings, in light of current and emerging therapeutics. These final insights are particularly formulated in the paradigmatic case of hematopoiesis and acute leukemia, which is one of the best known malignancies for which it is always hard, in presence of a clinical and histological remission, to decide between cure and dormancy of a tumoral clone.
\end{abstract}

\footnotetext{
This work is supported by ALMA3 project on the 'Analysis of Acute Myeloid Leukemia' and iCODE-Institute project funded by the idex Paris Saclay.

${ }^{*}$ Corresponding author: walid.djema@inria.fr

${ }^{1}$ W. Djema is with Inria Saclay, CentraleSupélec, Univ. Paris-Saclay \& with Inria SophiaAntipolis, Biocore and McTao teams, Université Côte d'Azur (UCA), France. walid.djema@inria.fr

${ }^{2}$ C. Bonnet and F. Mazenc are with Inria Saclay, Disco team, Université Paris-Saclay, CentraleSupélec, L2S (CNRS), France. catherine.bonnet@inria.fr, frederic.mazenc@inria.fr

${ }^{3} \mathrm{~J}$. Clairambault is with Inria, Mamba team and with Sorbonne Université, Paris 6, UPMC, Laboratoire Jacques-Louis Lions, Paris, France. jean.clairambault@inria.fr

${ }^{4}$ E. Fridman is with the Department of Electrical Engineering and Systems at the School of Electrical Engineering, Tel-Aviv, Israel. emilia@eng.tau.ac.il

${ }^{5}$ P. Hirsch and F. Delhommeau are with Sorbonne Université, GRC n7, Groupe de Recherche Clinique sur les Myéloproliferations Aiguës et Chroniques, AP-HP, Hôpital Saint-Antoine, Paris, F-75012, France. pierre.hirsch@aphp.fr, francois.delhommeau@aphp.fr
} 
Keywords: Delay, Nonlinear, Lyapunov, Cancer dormancy, Modeling.

2010 MSC: 93C23, 93C10, 93A30, 93D05, 92B05, 92C50, 80A30, 37L15, 39A30, $39 \mathrm{~A} 60$.

Highlights (to appear before the abstract in JTB template)

- Modeling the cell cycle and population cell dynamics taking into account: coexistence between normal and mutated stem cells, overproliferation of cancer stem cells and sensitivity to the immune system.

5 SC subpopulation, acquires self-renewing and proliferating capabilities similar to those of SCs $([28], 67])$. These stem-like cells are very often out of control [76] and they are capable of initiating, developing and regenerating cancers [28], hence their designation as cancer stem cells (CSCs) [49]. Very often, CSCs are 30 characterized by unhealthy behaviors such as excessive proliferation and loss of their differentiation faculties. This is what we observe for instance in the case 
of leukemia [25]. On the other hand, it cannot be disregarded that in some cases (as in breast cancer and leukemia [6, 26]) CSCs do not overproliferate (cancer without disease [35, or, in situ tumor). However, even during their non-overproliferating states, CSCs remain in general distinguishable through specific markers on their surface ${ }^{6}$ [76]. In medical research, the CSC hypothesi:7 postulates that one subpopulation of cells holds the power of initiating and regenerating cancer [28]. This stemness property in non-SCs has been first observed in leukemia, then in many other types of cancer. Not surprisingly, the study of leukemic cells became a model for many other stem-like cells [76].

\subsection{Evidences and underlying assumptions about cancer dormancy}

Strong evidence about the existence of a stalled growth state, commonly referred to as tumor dormancy, has been established many years ago when microscopic tumors were frequently encountered during autopsy examinations

45 (35], 64]). The most likely explanations (see [3], and also [35] and [80]) of CSCs dormancy state are: (H.1) blood and nutrient supply issues that prevent tumor growth, or at least delay its clinical manifestation [63], and (H.2) vigilance of the immune system which, in some cases, suffices to stop tumor development (see $32,63,80,90,93$ and the references therein). In fact, there has been a lengthy debate on the role of the immune system in the defense against cancer: a process called cancer immunosurveillance [90. The ambiguity about the immunosurveillance concept stems from the fact that often the immune system favors the development of the tumor instead of trying to eliminate it. The concept that attempts to integrate the diverse effects of the immune system on tumor progression is known as cancer immunoediting (see the review articles [80] and [90]). Even if it appears as an unsystematic process, an inter-

\footnotetext{
${ }^{6}$ For instance, stems cells in acute myeloid leukemia have some interleukin-3receptor $\alpha$ chain surface markers, which are not found in normal hematopoietic stems cells (see [31, 49]).

${ }^{7}$ The reference as CSC paradigm has also gained ground recently. Several subpopulations of cells, with distinct cancer-initiating powers, form actually a tumor. One subpopulation has an indefinite potential of self-renewing and shows stem-like status. It appears also that stemness might be a transient cell state that is associated to epigenetic changes [17.
} 
est arises for cancer therapies that are immuno-oriented, bearing the name of immunotherapy 8 . In a similar spirit, monoclonal antibodies, e.g. gemtuzumabozogamicin, have been approved in the treatment protocols of some cancers (as in acute myeloid leukemia [40]), even if more trials are still needed to identify their exact benefits [40, 78. Other cancer therapies, sometimes assimilated to immunotherapy, are using some immune checkpoint inhibitors (see for instance, [15], 55] and [66]). In the last part of our work, we will be shortly adopting some of these immuno-oriented concepts, associated with classical chemotherapy or targeted therapies, as it is frequently adopted in practice. More generally, the complex link between the immune system and cancer dormancy (as it is summarized in Fig. 3 of [80]) is implicitly represented in our model thanks to an extra-parameter that we introduce, as detailed in the sequel (see Section 2.2.

\subsection{Is cancer dormancy a promising therapeutic option?}

In a general perspective, apart from the interpretation of tumor dormancy as an observed natural phenomenon in human cancers, the idea to transform cancer into a chronic disease is in the voices of many people in the medical world nowadays [4, 39]. Indeed, the interesting issue here is about: how can we bring CSCs from an overproliferating activity to a dormant state? More precisely, since cancer treatments most often consist of delivering the maximum tolerable doses of drugs in order to kill clinically apparent tumors, and knowing that an incompletely eradicated cancer frequently grows again, even more aggressively than the initial one [28, the option of maintaining the tumor in dormancy is more appealing than trying to eradicate it [48]. Further discussions on the opportunities offered by cancer dormancy in therapeutics can be found for instance in [4], 39], 89] and the references therein.

The development of a relevant mathematical framework appears as a necessary tool to apprehend tumor dormancy as a biological mechanism [51, with

\footnotetext{
${ }^{8}$ Immunotherapy aims to help the immune system destroy cancer cells. It is given after or at the same time as - another cancer treatment such as chemotherapy.
} 
the ultimate goal to apply it in therapeutic settings. However, the task of mastering CSCs, i.e. bringing them into a dormant state, seems to be difficult to achieve. Indeed, one of the first dormancy-oriented therapeutic approaches in the case of solid tumors has not been very fruitful. It was based on the use of angiogenesis inhibitors 9 as drugs that choke off the blood supply of the tumor, in order to maintain it in dormancy. However, unexpected effects occurred in practice, and in some situations, targeting the blood vessels that feed tumors actually accelerated the spread of cancer (see 43, 77]). Therefore, it seems that tumor dormancy is more likely to be assigned to immuno-vigilance 10 (H.2), than to nutrient supply limitations $(\boldsymbol{H . 1})$. In light of the previously mentioned observations, one can say that dormancy has actually generated more issues than answers, in the process of understanding cancer. Among the open issues, we emphasize the following ones: when a treatment protocol is elaborated for CSCs eradication with a given rate of success, how can we actually administer it (or a part of it) in order to achieve dormancy? In addition, since eradication techniques may generate some surviving tumors which become even more aggressive than the initial ones, a key question is to determine whether it is effective to consider the same targets and drugs, as for CSCs eradication, in order to achieve dormancy? One can already figure out the utility of mathematical studies in such a context. Finally, we emphasize that, in the clinic of cancers today, eradication of CSCs remains the predominant treatment approach (although there is still a long way to improve the existing eradication treatment strategies [85]).

\subsection{Objectives of the study-Particular insights into the hematopoietic system}

We aim to provide a consistent theoretical framework for the modeling and the analysis of healthy and unhealthy cell dynamics, following different medical orientations, among which: the case where therapy aims to eradicate cancer

\footnotetext{
${ }^{9}$ These are substances that inhibit the growth of new blood vessels 35

${ }^{10}$ In particular, cancer dormancy results from the action of adaptive immunological mechanisms, through T cells, IL-12 and IFN-gamma 80.
} 
cells while preserving healthy ones, and the scenario that consists in maintaining healthy and unhealthy cells in a controlled stable steady-state (i.e. cancer dormancy). To that purpose, a model of coexistence between ordinary and mutated cells is introduced and analyzed. Firstly, we investigate the stability properties of the trivial steady state of the resulting model: this is equivalent to the radical case in which all the cells are eradicated. Then, we perform a stability analysis that applies to cases of cancer dormancy and unhealthy cell eradication (while healthy cells survive). For the biological motivations stated in the above sections, we will focus on the study of cancer dormancy throughout our paper.

At this juncture, we express our keen interest in the hematopoietic system. We define hematopoiesis as the process initiated by the hematopoietic SC population inside the bone marrow, that leads to the formation and continuous replenishment of all the blood cells in the body [45. Hematopoiesis provides a model for studying and understanding all the mammalian stem cells and their niches [18, as well as all the mechanisms involved in the cell cycle, particularly cell differentiation. The hematopoietic paradigm is used in biology and medicine, as well as in the modeling and analysis of all similar processes. In [72], the author reviewed the mathematical modeling of blood cell dynamics 130 and its related pathological disorders within the past five decades. It is within this framework that we can situate our work, as a continuity of modeling and stability analysis of blood cell dynamics. However, as for the majority of works discussed in [72], the models that we study can be used to cover other tissues and mechanisms. At this point, it is worth mentioning that pioneering works that formulated early blood cell dynamical models have been introduced for any type of cells [81], or borrowed from models describing other tissues, different from blood cell dynamics (see [16] for a dorsal epidermis cell model that inspired all the cell cycle models containing a resting phase). The interested reader is referred to 72 for more information. Therefore, we emphasize in this study the paradigmatic case of hematopoietic SCs, which are at the root of the overall hematopoietic system. Hematopoietic SCs give rise to both the myeloid 
and lymphoid lineages of blood cells. The myeloid cells include many types of white blood cells (monocytes, macrophages, neutrophils, eosinophils), red blood cells (erythrocytes), and platelets (megakaryocytes). The hematopoietic process has to be well controlled 45 in order to avoid a wide range of blood disorder ${ }^{11}$ Acute myeloid leukemia (AML) is one of the most deadly blood malignancies. It affects the myeloid lineage and it is characterized by an overproliferation of abnormal immature white blood cells (blasts) of the myeloid lineage. Currently, AML treatment still relies on heavy chemotherapy with a high toxicity level and a low rate of success 25. In fact, the only certain AML cure being not the result of chemotherapy, but of total bone marrow transplant that induces nearly $10-20 \%$ of mortality during the manipulation and due to severe reaction, GVH, of the graft versus the host. A better understanding of the behavior of CSCs (called leukemic cells in AML) should allow us to propose some selective combined targeted therapies that lead, theoretically, to cancer dormancy. In particular, our ambition is to provide a theoretical framework, taking into account observations made by hematologists, and allowing for the suggestion of insights into cancer treatments. It is in this light that we proposed in [23] a model of cohabitation between ordinary and mutated cells in the case of the hematopoietic system. The latter model follows recent observations (made in [44 and in many other works) that associate the emergence of leukemic cells with an accumulation of several mutations, most often occurring in a standard chronological order [44, in the SC compartment. Thus, we have mathematically analyzed in 23] and 24 two categories of heterogeneous cells as illustrated in 165 Figure 1 below, where the addition of mutations (on TET2, NPM1, FLT3) that we have considered had been established in [44. We pursue in this work an analysis that provides a theoretical framework following different medical orientations, among which: (i) the case where therapy aims to eradicate cancer cells while preserving healthy cells, (ii) a less demanding, more realistic, sce-

\footnotetext{
${ }^{11}$ In particular, periodic diseases, such as cyclic neutropenia and some cases of chronic acute leukemia (see [13], 19], [58, [73, and the references therein), but also overproliferating malignant hemopathies, such as acute myeloid leukemia ([2], [23], 65]).
} 

stable steady-state (cancer dormancy). Thus, our work extends the one that we proposed in [23, 24] and in a series of works: [1], [8], 9], (but see also [2], [21, 33], 38, 60], 65], 73], 83, and [84]). It is worth mentioning that the model in [9] can neither model dormancy nor the abnormal overproliferation

175 (e.g. invasion of the bone marrow by blasts). The latter point is improved by adopting a different form of fast self-renewing process, which has been recently introduced in [1, and where a subpopulation of cells is considered to be always active in proliferation [1]. In fact, cancer dormancy has not been considered in all the previously mentioned works. This is indeed a new area in cancer therapy (see [4, 28, 48, 89]) that we want to highlight here (but see also [51] for a different approach of modeling and analysis of cancer dormancy).

\subsection{Organization of the work}

In light of the above mentioned remarks, the coupled model (between healthy and mutated cells as in Figure 1 below) of interest is presented in Section 2 . Next, some features of the resulting coupled differential-difference model, together with the conditions of existence of our favourable steady states (reflecting dormancy and CSCs eradication), are discussed in Section 3. Then, in Section 4. the stability analysis of the case of all-cell extinction, via a construction of a linear Lyapunov-like functional, is performed (here we provide conditions for global exponential stability of the trivial steady state of the coupled model). Then, afterwards, we address in Section 5 the stability analysis, in the timedomain framework, of the cases describing cancer dormancy, and, unhealthy cells eradication (while healthy cells survive). The latter study goes through quadratic Lyapunov-like constructions (i.e. suitable degenerate functionals for 195

the class of differential-difference systems). In fact, we are going to use two slightly different constructions: the first one is more general and relies on Linear Matrix Inequality (LMI) conditions derived via the descriptor method [37], applied to the linear approximation of the model around its nontrivial steady state of interest. This approach aims to provide a theoretical (sufficient) sta- 


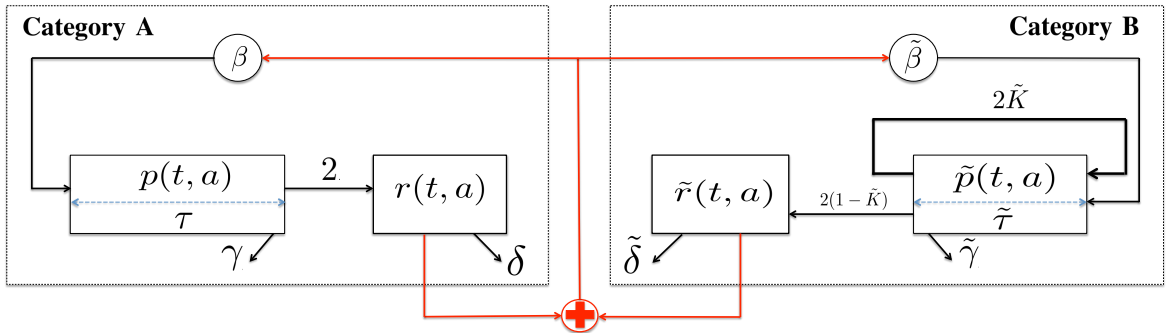

Figure 1: Schematic representation of the coupled model of interest, involving a healthy SCs compartment (on the left) and an unhealthy compartment (on the right). For the sake of simplicity, we assume that unhealthy cells are those presenting mutations that lead to cancer. Indeed, we consider that abnormal stem cells (Category B) have for instance a first mutation in some genes encoding enzymes in epigenetics (e.g. on TET2, DNMT3A 20] 71]), that increases the self-renewing activity of the affected cells. A more serious pathological situation arises when a second mutation, affecting this time the pathways regulating the differentiation process such as NPM1 or transcription factors, appears on some of the cells. The superposition of these two events yields a blockade in differentiation. Finally, a subsequent mutation impairing proliferation control (e.g. FLT3-ITD) appears in a subpopulation of cells that have already accumulated one or more of the previously mentioned mutations. The latter event activates an uncontrolled overproliferation of a subpopulation of cells (CSCs) and thereby causes AML [44]. Throughout this work, with a kind of abuse of notation, we use equivalently the designations: unhealthy cells, mutated cells, and CSCs. Similarly, healthy cells (Category A), or ordinary cells, represented on the left, are those which do not have any abnormal mutation, or those presenting some abnormalities which are not related to cancer. The definitions of the biological parameters $(\delta, \tilde{\delta}, \gamma, \tilde{\gamma}, \ldots)$ are provided in Section 2 


\section{A new general mathematical model involving coexistence between healthy and cancer stem cells}

Our objective is to introduce a model more general than the existing ones, with regard to the recent biological features of interest, that are: cancer dormancy [26, 28], control and eradication of CSCs [48]. In particular, the compartment of unhealthy cells is hierarchized according to the severity of the mutations: cells that accumulate mutations up to that of FLT3 duplication (see Figure 1) are constantly active in proliferation (as in [1). Our configuration allows us to reproduce and interpret the case of cancer dormancy, with the ultimate goal of providing theoretical stability conditions, along with therapeutic insights, that lead to stable dormant CSCs.

\subsection{A multi-compartmental general model for healthy and unhealthy cells}

We focus on the model illustrated in Figure 1, where CSCs are characterized by an ability to over-proliferate represented by the parameter $\tilde{K}$ (in days ${ }^{-1}$ ), 225 as considered in [1, and previously envisaged in 9] in a different configuration. More precisely, we notice that a subpopulation of unhealthy cells is in a permanent dividing state, namely the portion corresponding to $2 \tilde{K}$, where, $0<\tilde{K}<1$ (as in 1] for a non-coupled model), which is different from the healthy SCs behavior (Figure1, on the left), where daughter cells, that arise from division of

230 healthy mother cells, leave the proliferating compartment and join necessarily the resting one. Healthy resting stem cells can stay in $G_{0}$ until their death, differentiate, or start a new proliferating cycle by being transferred through the reintroduction function $\beta$ to there proliferating compartment. Indeed, we mention that as many other works (see [2, 57, 73, among others), we are considering a compartmental model in which each cell can be in a resting phase or in a proliferating one. Finally, we mention that the coupled models studied in [9, 23] do not admit a stable steady state that describes cancer dormancy, and this is an issue that we overcome here by considering a more general manner of coupling healthy and unhealthy SCs as discussed in the sequel. 
Next, we denote by $\delta$ (resp. $\tilde{\delta}$ ) the rate, expressed in $d_{a y s^{-1}}$, of resting cells, which is lost either by differentiation or natural cell death for healthy SCs (resp. CSCs). A resting cell may start a cell cycle by entering in the proliferating phase during which each proliferating SC (resp. CSC) may die by apoptosis at a rate, expressed in days $^{-1}, \gamma($ resp. $\tilde{\gamma})$, or complete its mitosis and become two daughter cells at the end of the proliferating phase. We denote $\tau$ (resp. $\tilde{\tau}$ ) the average time (in days) taken to complete mitosis in the healthy (resp. unhealthy) proliferating compartment. For proliferation, the mechanisms regulating the entry into the cell cycle - at the cellular level - rely on some regulatory molecules that can play the role of growth factors (by stimulating the entry into proliferation of resting healthy and unhealthy cells), or, they can play the role of mitotic inhibitor ligands (meaning that mitosis proceeds normally if inhibitors are not combined with cell receptors, while it is stalled when they bind them). Consequently, we consider in our model that the transfer from the resting to the proliferating states is controlled by some reintroduction functions (as in 255 [57, 73] and the majority of earlier works). More precisely, we let $\beta$ (resp. $\tilde{\beta}$ ) be the reintroduction function from the healthy (resp. unhealthy) resting phase to the healthy (resp. unhealthy) proliferating phase. In addition, since healthy and unhealthy cells share the same environment (called niches [18] in hematopoiesis), we consider that each of the two functions $\beta$ and $\tilde{\beta}$ depends simultaneously on both the total density of resting healthy cells, $x(t)=\int_{0}^{\infty} r(t, a) d a$, and the total density of unhealthy resting cells, $\tilde{x}(t)=\int_{0}^{\infty} \tilde{r}(t, a) d a$, where $r(t, a)$ and $\tilde{r}(t, a)$ are, respectively, the densities of resting healthy cells and resting unhealthy cells, of age $a \geq 0$, at time $t \geq 0[23$. This modeling approach reflects cohabitation between healthy and unhealthy cells, by considering that the entry into proliferation of healthy cells (resp. unhealthy cells) is also dependent on the total density of unhealthy cells (resp. healthy cells), the dynamics of the left and the right subpopulations in Figure 1 becoming thus strongly coupled. Thus, the choice of the arguments (i.e. coupling forms) given to the functions $\beta$ and $\tilde{\beta}$ is crucial, since these arguments quantify the regulating mechanisms that affect healthy and unhealthy cells (see [57] for the case of non-coupled models). 


\subsection{The coupling form between ordinary and mutated cells}

The functions $\beta$ and $\tilde{\beta}$ represent the physiological inhibitory hormonal feedback by Granulocyte Colony Stimulating Factors $(G-C S F)$ that is valid in the case of healthy as in the case of cancer cells. However, in the latter unhealthy situation, the sensitivity of the unhealthy cell population to this feedback may strongly vary. Now, the remaining issue regarding the functions $\beta$ and $\tilde{\beta}$ is to select the coupling function between the total density of healthy resting cells $x$ and the total density of mutated resting cells $\tilde{x}$ (i.e., to specify how $\beta$ and $\tilde{\beta}$ actually depend on $x$ and $\tilde{x}$ ). It appears that the simplest choice is to consider that both $\beta$ and $\tilde{\beta}$ depend on the sum $x+\tilde{x}$, as previously considered in 9 . and 23 . The latter scheme expresses a kind of absence of dominance between the populations $x$ and $\tilde{x}$, since they show equal influence on $\beta$ and $\tilde{\beta}$. However, differences actually exist between $x$ and $\tilde{x}$ in their shared host - in particular immune - environment, mainly due to the mutations acquired by abnormal cells [46] and the reaction of the immune system. Changes that occur in mutated cell behavior may enhance the growth of cancer and result in cachexia and death [12] (see also [27, 70] for biological observations and modeling of the interaction between cancer and host environment). In our modeling approach, considering a coupling in the form $x+\tilde{x}$ means equal sensitivity of ordinary and mutated resting populations regarding the diverse proliferation regulation mechanisms, that act on the reintroduction of resting cells into proliferation. For example, due to epigenetic mutations, unhealthy cells may become less sensitive than healthy ones to the regulatory molecules secreted by the body and avoid the immune system (i.e. an immunosuppressive effect); on the other hand, healthy cells are in turn insensitive to the action of the immune system and less sensitive to drugs, since these drugs are designed to target unhealthy cells. In summary, healthy and unhealthy cells may react differently to their shared host environment (see Figure 2 below), which may result in the dominance of one subpopulation (healthy or unhealthy), or possibly in cancer dormancy [80]. Our first objective is to achieve a model that reproduces all these situations. Thus, we aim here to extend the modeling aspects by considering a more general form 
of coupling functions, so that some immunological effects can be represented. For that purpose, we consider that the argument of $\beta$ is $x+\tilde{x}$, while $\tilde{\beta}$ depends at the same time on a weighted combination $x+\tilde{\alpha} \tilde{x}$, where $\tilde{\alpha}$ is some positive constant. We will show later in Section 3 that actually dormancy may be found mostly when $\tilde{\alpha} \neq 1$. In an illustrative manner, Figure 2 provides a representation of the cases:

- $0 \leq \tilde{\alpha} \leq 1$ : even if ordinary and mutated cells are sharing the same environment, the mutated ones are less sensitive to the regulatory system present in the host environment, that may be identified as effects of the immune system on mutated cell proliferation. Consequently, unhealthy cells may escape a part of the regulatory system, including the immune system. This appears to be in line with medical practice, since the unhealthy behavior is mainly due to genetic/epigenetic mutations that make cells partially unresponsive to the regulating system. Consequently, the case $0 \leq \tilde{\alpha} \leq 1$ suits well the untreated unhealthy behavior, in which cells avoid immune attacks and tend to get out of control, possibly leading to outgrowth of CSCs $80,90,95$.

- $\tilde{\alpha}>1$ : this case can describe an environment where unhealthy cells are more affected by the regulatory molecules than the healthy ones. This may be partly due to the effector response of the immune system (cancer immunosurveillance [90]), which may explain the dormancy phenomenon as a result of an efficient immune action that contains cancer growth 93 . In other words, the case $\tilde{\alpha}>1$ stands for a situation where proliferation of unhealthy cells may be locally kept under check by the immune system. This is the role of the innate and adaptive immunity which may lead to extrinsic tumor suppression in some rare cases, or to the adaptive immunity ( $\mathrm{T}$ cells, IL-12, IFN-gamma) that at least may maintain cancer dormancy for long time 80 .

Remark 1. A concept of dominance between healthy and mutated cells results from $\tilde{\alpha}$, that allows for an implicit representation of the immunologic mechanisms. In fact, what really makes the difference between cells is their respective sensitivity to the immune environment. The natural feedback represented by the 
functions $\beta$ and $\tilde{\beta}$ depending on their arguments $x$ and $\tilde{x}$, is in the case of cancer cells tuned by a sensititvity parameter $\tilde{\alpha}$ that may be seen as the faculty of unhealthy cells to over-express $(\tilde{\alpha}>1)$ or hide $(\tilde{\alpha}<1)$ their surface antigens.

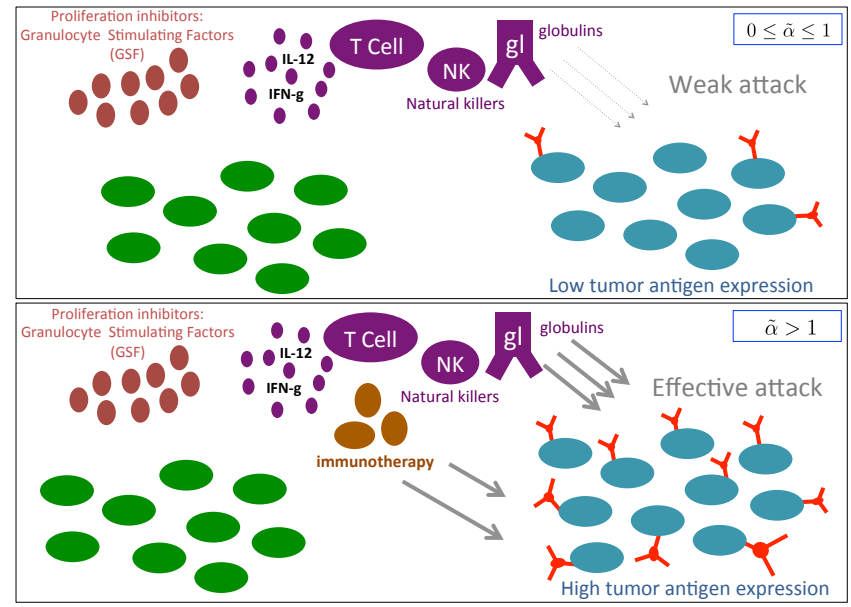

Figure 2: Cartoon illustration of healthy and unhealthy cells in their shared environment. Ordinary SCs with normal behavior are in green, while mutated ones that go through quiescence to re-start a cell cycle (i.e. not the ones having the FLT3 mutation that makes them constantly active into proliferation) are in blue. The regulation of cell proliferation may include different mechanisms: release of growth factors and mitotic regulatory molecules, $\mathrm{T}$ cells, natural killers, globulins, IFN-g (IFN-gamma) and IL-12 (interleukin 12). Epigenetic mutations may also play a role on the way cells react to the whole regulating system. The case $0 \leq \tilde{\alpha} \leq 1$ fits well a situation in which unhealthy cells are less sensitive to proliferation regulation than healthy ones. In this case, abnormal cells may hide their tumor antigens (an immunosuppressive state), which can be also due to the tumor variant cells that become no longer recognized and attacked by the adaptive immunity 80 and grow into insensitive cells to the entire immune effector mechanism. This condition is not enough in itself for the development of cancer, but it certainly favors it and may lead to the escape phase. On the other hand, the case $\tilde{\alpha}>1$ represents a situation in which proliferation of unhealthy cells is more controlled than the one of healthy cells. Reasons for this include an effective action carried out by the innate and adaptive immunity (sometimes this action suffices for total tumor eradication, see e.g. 90 and Fig. 3 in 80$]$ ), but also the use of immunotherapy that acts in two ways: boosting the immune system to eliminate CSCs, and/or, enhancing the immune response by providing additional combative components such as reenabling exhausted $\mathrm{T}$ cells.

In addition, it can also be argued that $\tilde{\alpha}>1$ relies on the use of drugs (immunotherapy, chemotherapy, etc) that specifically target unhealthy cells. Indeed, we recall that immunotherapy mainly enhances the immune response, and that recent chemotherapy or targeted therapies are increasingly more accurate due to the overexpression of cancer receptors (which allow them to target 
unhealthy cells while the majority of healthy cells are spared). Finally, we mention that the introduction of the above considerations related to the coupling functions between $x$ and $\tilde{x}$ will make the dynamics of the resulting model richer than earlier models, as discussed in the next sections (Section 3). To the authors' knowledge, no equivalent model exists in the literature. Next, as conventionally considered, we assume that $\tilde{\beta}$ and $\beta$ are nonlinear continuous decreasing functions, and, $\lim _{\ell \rightarrow \infty} \tilde{\beta}(\ell)=\lim _{\ell \rightarrow \infty} \beta(\ell)=0$. As in [57, [73, and all subsequent works for non-coupled models, we consider the typical Hill forms:

$$
\tilde{\beta}(\ell)=\frac{\tilde{\beta}(0)}{1+\tilde{b} \ell^{\tilde{n}}}, \quad \beta(\ell)=\frac{\beta(0)}{1+b \ell^{n}}
$$

where $\tilde{b}, b, \tilde{\beta}(0)$ and $\beta(0)$ are strictly positive real numbers and, $\tilde{n} \geq 2$ and $n \geq 2$. In fact, the Hill functions in (1), that belong to the family of functions with negative Schwarzian derivatives (see [5], Chap. 3) are commonly encountered in many real-life problems. Classical arguments on cooperativity of enzyme inhibition kinetics (see Chap. 1 in [52, and [75]), allow to determine the Hilltype expressions (1). The cooperative effect results in general from the fact that the binding of one regulatory molecule on one extracellular (surface) receptor of one cell will affect the binding of subsequent regulatory molecules on other receptors of the same cell. Due to the above considerations on the different sensitivities between healthy and unhealthy cells in the niches ( 1 and $\tilde{\alpha} \neq 1$, respectively), we can readily deduce that for given total densities $x$ and $\tilde{x}$, the associated reintroduction functions $\beta$ and $\tilde{\beta}$ actually operate according to:

$$
\tilde{\beta}(x+\tilde{\alpha} \tilde{x})=\frac{\tilde{\beta}(0)}{1+\tilde{b}(x+\tilde{\alpha} \tilde{x})^{\tilde{n}}}, \quad \beta(x+\tilde{x})=\frac{\beta(0)}{1+b(x+\tilde{x})^{n}} .
$$

\subsection{Equations describing the dynamics of coupled cell populations}

After the description of the particular case of the reintroduction functions $\beta$ and $\tilde{\beta}$ according to the variation of the cell densities $x$ and $\tilde{x}$ (as in Figure 3), we now focus on the dynamical equations describing the populations of cells. 

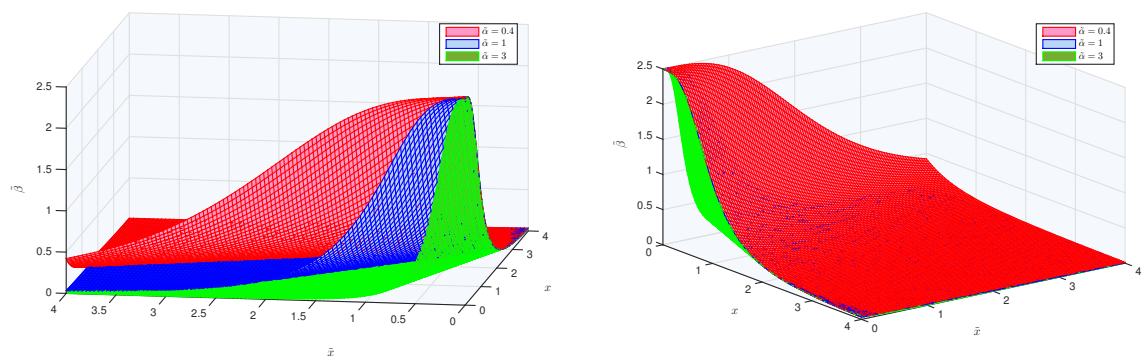

Figure 3: Illustrative example of variations of a typical $\tilde{\beta}$-surface with respect to $\tilde{x}$ and $x$, for different values of $\tilde{\alpha}$ (i.e. in the three possible situations: $\tilde{\alpha}>1, \tilde{\alpha}=1$, and $\tilde{\alpha}<1$ ).

Similarly to $x$ and $\tilde{x}$, we denote by $y$ and $\tilde{y}$, respectively, the total densities of proliferating healthy and unhealthy cells: $y(t)=\int_{0}^{\tau} p(t, a) d a$, and, $\tilde{y}(t)=$ $\int_{0}^{\tilde{\tau}} \tilde{p}(t, a) d a$. The age-structured PDEs describing the coupled model in Figure 1. are given for all $t>0$ by:

$$
\left\{\begin{array}{l}
\partial_{t} \tilde{r}(t, a)+\partial_{a} \tilde{r}(t, a)=-[\tilde{\delta}+\tilde{\beta}(x, \tilde{x}, \tilde{\alpha}, t)] \tilde{r}(t, a), \quad a>0, \\
\partial_{t} \tilde{p}(t, a)+\partial_{a} \tilde{p}(t, a)=-\tilde{\gamma} \tilde{p}(t, a), \quad 0<a<\tilde{\tau}, \\
\partial_{t} r(t, a)+\partial_{a} r(t, a)=-[\delta+\beta(x, \tilde{x}, t)] r(t, a), \quad a>0, \\
\partial_{t} p(t, a)+\partial_{a} p(t, a)=-\gamma p(t, a), \quad 0<a<\tau .
\end{array}\right.
$$

In McKendrick-type models ([33], [56], [91]), we observe that only the death rates $(\delta, \tilde{\delta}, \gamma$ and $\tilde{\gamma})$, and the removal terms ( $\beta$ and $\tilde{\beta}$, since the reintroduction functions are considered as cell loss from resting cells) appear in the PDE system (3). On the other hand, the new births, which are the renewal conditions at the age $a=0$, for resting and proliferating cells, are introduced through the following boundary conditions:

$$
\left\{\begin{aligned}
\tilde{r}(t, 0) & =2(1-\tilde{K}) \tilde{p}(t, \tilde{\tau}) \\
\tilde{p}(t, 0) & =\tilde{\beta}(x, \tilde{x}, \tilde{\alpha}, t) \tilde{x}(t)+2 \tilde{K} \tilde{p}(t, \tilde{\tau}) \triangleq \tilde{u}(t), \\
r(t, 0) & =2 p(t, \tau) \\
p(t, 0) & =\beta(x, \tilde{x}, t) x(t)
\end{aligned}\right.
$$

for all $t>0$, and where $\tilde{u}(t)$ represents the density of new proliferating unhealthy cells at time $t>0$ [1]. Finally, the initial age-distributions, respectively, 
$\tilde{r}(0, a)=\tilde{r}_{0}(a)$, for $a>0, \tilde{p}(0, a)=\tilde{p}_{0}(a)$, for $0<a<\tilde{\tau}, r(0, a)=r_{0}(a)$, for $a>0$, and $p(0, a)=p_{0}(a)$, for $0<a<\tilde{\tau}$, are assumed to be $L^{1}$-functions.

Now, inspired by an illustrative approach in [87, we give a biological explanation of the method of characteristics in our context. To avoid redundancy, we focus only on the unhealthy compartment. Let us define $\mathfrak{p}^{*}(a, s)$ as the density of unhealthy proliferating cells, of age $a$, that entered to the unhealthy proliferating phase at time $s$. This coincides with the density of unhealthy proliferating cells at time $t=a+s([87])$. In other words, $\mathfrak{p}^{*}(a, s)=\tilde{p}(a+s, a)$. Therefore,

$$
\frac{\partial \mathfrak{p}^{*}(a, s)}{\partial a}=\left.\frac{\partial \tilde{p}(t, a)}{\partial t}\right|_{t=a+s}+\left.\frac{\partial \tilde{p}(t, a)}{\partial a}\right|_{t=a+s}=-\tilde{\gamma} \mathfrak{p}^{*}(a, s)
$$

It follows that $\mathfrak{p}^{*}(a, s)=\mathfrak{p}^{*}(0, s) e^{-\tilde{\gamma} a}$, where $\mathfrak{p}^{*}(0, s)=\tilde{p}(s, 0)$.

Now, let us recover $\tilde{p}$ from $\mathfrak{p}^{*}([87])$. Noticing that $\tilde{p}(t, a)=\mathfrak{p}^{*}(a, t-a)$, for $t>a$, we obtain, $\tilde{p}(t, a)=e^{-\tilde{\gamma} a} \tilde{p}(t-a, 0)$, for all $t>a$.

Next, we define $\mathfrak{p}^{\mathfrak{v}}(t, s)=\tilde{p}(t, t+s)$, which can be interpreted as the density of unhealthy proliferating cells that are in the proliferating phase at time $t$, and have been in the proliferating phase at time 0 , with an age $a=s$ at $t=0$. Arguing as for $\mathfrak{p}^{*}([87])$, we find that $\frac{\partial \mathfrak{p}^{\mathfrak{v}}(t, s)}{\partial t}=-\tilde{\gamma} \mathfrak{p}^{\mathfrak{v}}(t, s)$.

Then, $\mathfrak{p}^{\mathfrak{v}}(t, s)=\mathfrak{p}^{\mathfrak{v}}(0, s) e^{-\tilde{\gamma} t}$, where $\mathfrak{p}^{\mathfrak{v}}(0, s)=\tilde{p}(0, s)=\tilde{p}_{0}(s)$. Recovering $\tilde{p}$ from $\mathfrak{p}^{\mathfrak{v}}$, for $a \geq t$, gives us $\tilde{p}(t, a)=e^{-\tilde{\gamma} t} \tilde{p}_{0}(a-t)$, for all $a \geq t$.

We deduce that we have recovered the well-known solution ([87]):

$$
\tilde{p}(t, a)= \begin{cases}e^{-\tilde{\gamma} t} \tilde{p}_{0}(a-t), & 0 \leq t \leq a \\ e^{-\tilde{\gamma} a} \tilde{p}(t-a, 0), & t>a .\end{cases}
$$

Consequently, the first equation in (4) is then equivalent to

$$
\tilde{r}(t, 0)= \begin{cases}2(1-\tilde{K}) e^{-\tilde{\gamma} t} \tilde{p}_{0}(\tilde{\tau}-t), & 0 \leq t \leq \tilde{\tau} \\ 2(1-\tilde{K}) e^{-\tilde{\gamma} \tilde{\tau}} \tilde{p}(t-\tilde{\tau}, 0), & t>\tilde{\tau}\end{cases}
$$

From biological considerations we set, $\lim _{a \rightarrow \infty} \tilde{r}(t, a)=\lim _{a \rightarrow \infty} r(t, a)=0$, for all fixed value of $t \geq 0$. Then, using (8), and by integrating the first equation in (3) with respect to $a$ between 0 and $+\infty$, we determine that the long time behavior $([1])$ of $\tilde{x}$ is given by $\dot{\tilde{x}}(t)=-(\tilde{\delta}+\tilde{\beta}(x, \tilde{x}, \tilde{\alpha}, t)) \tilde{x}(t)+2(1-\tilde{K}) e^{-\tilde{\gamma} \tilde{\tau}} \tilde{u}(t-\tilde{\tau})$, where we recall that the density $\tilde{u}(t)$ is the one defined in (4), and represents 
for all $t>0$ the density of new unhealthy proliferating cells. Similarly, by integrating the second equation in (3) over the variable $a$, between 0 and $\tilde{\tau}$, and using $\tilde{p}(t, \tilde{\tau})=\tilde{u}(t-\tilde{\tau})$, we get $\dot{\tilde{y}}(t)=-\tilde{\gamma} \tilde{y}(t)+\tilde{\beta}(x, \tilde{x}, \tilde{\alpha}, t) \tilde{x}(t)-(1-2 \tilde{K}) e^{-\tilde{\gamma} \tilde{\tau}} \tilde{u}(t-\tilde{\tau})$. Similarly, we can check that for the healthy compartment, we obtain for all $t>0$ :

$$
p(t, a)= \begin{cases}e^{-\gamma t} p_{0}(a-t), & 0 \leq t \leq a \\ e^{-\gamma a} p(t-a, 0), & t>a .\end{cases}
$$

It follows that the third equation in (4) is then equivalent to:

$$
r(t, 0)= \begin{cases}2 e^{-\gamma t} p_{0}(\tau-t), & 0 \leq t \leq \tau, \\ 2 e^{-\gamma \tau} p(t-\tau, 0), & t>\tau,\end{cases}
$$

where $p(t-\tau, 0)$ is deduced from the fourth equation in (4). Thus, using similar arguments as for the unhealthy compartment, we deduce the following overall system for all $t>0$,

$$
\left\{\begin{aligned}
\dot{\tilde{x}}(t) & =-[\tilde{\delta}+\tilde{\beta}(x, \tilde{x}, \tilde{\alpha}, t)] \tilde{x}(t)+2(1-\tilde{K}) e^{-\tilde{\gamma} \tilde{\tau}} \tilde{u}(t-\tilde{\tau}), \\
\dot{\tilde{y}}(t) & =-\tilde{\gamma} \tilde{y}(t)+\tilde{\beta}(x, \tilde{x}, \tilde{\alpha}, t) \tilde{x}(t)-(1-2 \tilde{K}) e^{-\tilde{\gamma} \tilde{u}} \tilde{u}(t-\tilde{\tau}), \\
\tilde{u}(t) & =\tilde{\beta}(x, \tilde{x}, \tilde{\alpha}, t) \tilde{x}(t)+2 \tilde{K} e^{-\tilde{\gamma} \tilde{u}} \tilde{u}(t-\tilde{\tau}), \\
\dot{x}(t) & =-[\delta+\beta(x, \tilde{x}, t)] x(t)+2 e^{-\gamma \tau} \beta(x, \tilde{x}, t-\tau) x(t-\tau), \\
\dot{y}(t) & =-\gamma y(t)+\beta(x, \tilde{x}, t) x(t)-e^{-\gamma \tau} \beta(x, \tilde{x}, t-\tau) x(t-\tau) .
\end{aligned}\right.
$$

We notice that the dynamics of $x, \tilde{x}$ and $\tilde{u}$ do not depend on $y$ and $\tilde{y}$. This (triangular) system structure leads us to study first:

$$
\left\{\begin{array}{l}
\dot{\tilde{x}}(t)=-[\tilde{\delta}+\tilde{\beta}(x(t)+\tilde{\alpha} \tilde{x}(t))] \tilde{x}(t)+2(1-\tilde{K}) e^{-\tilde{\gamma} \tilde{\tau}} \tilde{u}(t-\tilde{\tau}), \\
\tilde{u}(t)=\tilde{\beta}(x(t)+\tilde{\alpha} \tilde{x}(t)) \tilde{x}(t)+2 \tilde{K} e^{-\tilde{\gamma} \tilde{\tau}} \tilde{u}(t-\tilde{\tau}), \\
\dot{x}(t)=-[\delta+\beta(x(t)+\tilde{x}(t))] x(t)+2 e^{-\gamma \tau} \beta(x(t-\tau)+\tilde{x}(t-\tau)) x(t-\tau) .
\end{array}\right.
$$

We can prove that a unique piecewise continuous solution, $(\tilde{x}(t), \tilde{u}(t), x(t))$, exists for all $t \geq 0$, when system (10) is associated with appropriate initial conditions $\left(\varphi_{\tilde{x}}, \varphi_{\tilde{u}}, \varphi_{x}\right)$, where, $\varphi_{\tilde{x}} \in \mathcal{C}([-\tau, 0], \mathbb{R}), \varphi_{x} \in \mathcal{C}([-\tau, 0], \mathbb{R})$, and, $\varphi_{\tilde{u}} \in \mathcal{C}([-\tilde{\tau}, 0], \mathbb{R})$. Moreover, we can show that the system 10$]$ is positive, since $\tilde{K} \in(0,1)$. Throughout this work, only positive solutions of 10 are considered. 


\section{Notable features of the coupled model}

In this section, we point out some properties of the model 10 that highlight its rich dynamics, according to the following possibly existing cases 12

\begin{tabular}{lccccc}
\hline Point of interest of $\tilde{x}$ & 0 & $\tilde{x}_{e}$ & 0 & $\tilde{x}_{e}$ & $+\infty$ \\
\hline Point of interest of $\tilde{u}$ & 0 & $\tilde{u}_{e}$ & 0 & $\tilde{u}_{e}$ & $+\infty$ \\
\hline Point of interest of $x$ & 0 & 0 & $x_{e}$ & $x_{e}$ & $*$ \\
\hline
\end{tabular}

Cell extinction: clearly, $(0,0,0)$, is an equilibrium point of model 100 . Biologically, convergence to zero is synonymous of the extinction of all the cells (both healthy and unhealthy populations). From a therapeutic standpoint, we said that we aim to address theoretical studies for the case of unhealthy cells eradication (while ensuring that healthy cells survive), and also for a dormancy steady state (where all the cells are in a stable steady state). In both situations we do not want that healthy cells vanish. However, chemotherapy always affects - to a certain extent - healthy cells. But side effects of recent chemotherapy treatments are fewer than those of the drugs used in the past, since novel molecules (targeted therapies) are designed to target overexpressed receptors corresponding to mutated cells (i.e. drugs are more accurate since they attack cells with specific extracellular receptors expressed only on mutated cells). In addition, medications are mainly acting on cells during their phase of proliferation, while it appears that most of the healthy cells are in quiescence. Therefore, we consider that only a radical therapy will lead to total cell eradication, and this is a situation that we want to avoid. Nevertheless, the theoretical conditions, depending on the biological functions and parameter involved in our model, that cause total cell eradication are discussed in Section 4.

Escape from dormancy in diseased cells: one of the main concerns related to dormancy is to explain how escape from tumor dormancy can emerge (see [51] and the non-coupled model in [1] that admits unbounded solutions).

\footnotetext{
${ }^{12}$ Here we are considering that $\tilde{x}_{e}>0, \tilde{u}_{e}>0$ and $x_{e}>0$. We can notice that $\tilde{x}_{e}=0$ implies that $\tilde{u}_{e}=0$, and vice versa.
} 
Similarly to [1, we notice in the coupled model (10) that the CSC-compartment cancer. Indeed, from the second equation in 10 it is obvious that $2 \tilde{K} e^{-\tilde{\gamma} \tilde{\tau}}>1$, implies that, $\lim _{t \rightarrow+\infty} \tilde{u}(t)=+\infty$. It follows from the first equation in 10 that $\lim _{t \rightarrow+\infty} \tilde{x}(t)=+\infty$. This situation may reflect the escape from tumor dormancy, or the invasion of the bone marrow by the blasts as in AML.

$\square$ Existence of the desired steady states $\mathfrak{D}$ and $\mathfrak{E}$ : let us start from the general case in which the point $\left(\tilde{x}_{e}, \tilde{u}_{e}, x_{e}\right)$ is a nonnegative steady state of (10). Therefore, it follows that this equilibrium point satisfies:

$$
\left\{\begin{array}{l}
{\left[\tilde{\delta}+\tilde{\beta}\left(x_{e}+\tilde{\alpha} \tilde{x}_{e}\right)\right] \tilde{x}_{e}=2(1-\tilde{K}) e^{-\tilde{\gamma} \tilde{\tau}} \tilde{u}_{e}} \\
\tilde{\beta}\left(x_{e}+\tilde{\alpha} \tilde{x}_{e}\right) \tilde{x}_{e}=\left(1-2 \tilde{K} e^{-\tilde{\gamma} \tilde{\tau}}\right) \tilde{u}_{e} \\
{\left[\delta-\left(2 e^{-\gamma \tau}-1\right) \beta\left(x_{e}+\tilde{x}_{e}\right)\right] x_{e}=0}
\end{array}\right.
$$

385 suming that: $2 \tilde{K} e^{-\tilde{\gamma} \tilde{\tau}}<1$. Indeed, our main objective here is to determine necessary and sufficient conditions for the existence of $\mathfrak{D}=\left(\tilde{x}_{e}, \tilde{u}_{e}, x_{e}\right)$, where $x_{e}>0, \tilde{x}_{e}>0$ and $\tilde{u}_{e}>0$, and for the existence of $\mathfrak{E}=\left(0,0, x_{e}\right)$, where $x_{e}>0$.

First, since $\beta$ is continuous and decreasing from $\beta(0)$ to zero, we deduce from the third equation in 11 that,

$$
\delta<\left[2 e^{-\gamma \tau}-1\right] \beta(0)
$$

is a necessary and sufficient condition for the existence of $x_{e}$ and $\tilde{x}_{e}$ such that, ${ }_{390} x_{e}+\tilde{x}_{e}>0$, and, $\delta-\left(2 e^{-\gamma \tau}-1\right) \beta\left(x_{e}+\tilde{x}_{e}\right)=0$. In fact, the inequality 12 is a necessary and sufficient condition for the existence of $\mathfrak{E}$ (but not $\mathfrak{D}$ ).

Next, from the second equation in 111 , we obtain that $\tilde{u}_{e}=\frac{\tilde{\beta}\left(x_{e}+\tilde{\alpha} \tilde{x}_{e}\right) \tilde{x}_{e}}{1-2 \tilde{K} e^{-\tilde{\gamma} \tilde{\tau}}}$.

By substituting $\tilde{u}_{e}$ in the first equation of (11), we get:

$$
\left[\tilde{\delta}-\frac{2 e^{-\tilde{\gamma} \tilde{\tau}}-1}{1-2 \tilde{K} e^{-\tilde{\gamma} \tilde{\tau}}} \tilde{\beta}\left(x_{e}+\tilde{\alpha} \tilde{x}_{e}\right)\right] \tilde{x}_{e}=0
$$


The fact that $\tilde{\beta}$ is continuous and decreasing implies that the condition,

$$
\tilde{\delta}<\left[\frac{2 e^{-\tilde{\gamma} \tilde{\tau}}-1}{1-2 \tilde{K} e^{-\tilde{\gamma} \tilde{\tau}}}\right] \tilde{\beta}(0)
$$

is necessary and sufficient for the existence of $x_{e}$ and $\tilde{x}_{e}$, such that, $x_{e}+\tilde{\alpha} \tilde{x}_{e}>0$, and, $\tilde{\delta}-\frac{2 e^{-\tilde{\gamma} \tilde{\tau}}-1}{1-2 \tilde{K} e^{-\tilde{\gamma} \tilde{\tau}}} \tilde{\beta}\left(x_{e}+\tilde{\alpha} \tilde{x}_{e}\right)=0$. Obviously, we notice that, $2 \tilde{K} e^{-\tilde{\gamma} \tilde{\tau}}<1<$ $2 e^{-\tilde{\gamma} \tilde{\tau}}$. In fact, the condition (14) is a necessary and sufficient condition for the existence of $\left(\tilde{x}_{e}, \tilde{u}_{e}, 0\right)$, where $\tilde{x}_{e}>0$ and $\tilde{u}_{e}>0$.

It is worth mentioning that, if the condition 12 is satisfied (i.e. the necessary and sufficient condition for the existence of $\mathfrak{E})$, together with the condition

$$
\tilde{\delta}>\left[\frac{2 e^{-\tilde{\gamma} \tilde{\tau}}-1}{1-2 \tilde{K} e^{-\tilde{\gamma} \tilde{\tau}}}\right] \tilde{\beta}(0),
$$

then $(0,0,0)$ and $\mathfrak{E}$ are the unique existing steady states of the studied model. Let us now focus on the case where both $x_{e}$ and $\tilde{x}_{e}$ are simultaneously strictly positive (and then $\tilde{u}_{e}$ is strictly positive). In the latter situation, we get,

$$
\left\{\begin{array}{l}
x_{e}+\tilde{\alpha} \tilde{x}_{e}=\tilde{\beta}^{-1}(\tilde{\mu}), \\
x_{e}+\tilde{x}_{e}=\beta^{-1}(\mu),
\end{array}\right.
$$

where, $\mu=\frac{\delta}{2 e^{-\gamma \tau}-1}$, and, $\tilde{\mu}=\frac{\tilde{\delta}\left(1-2 \tilde{K} e^{-\tilde{\gamma} \tilde{\tau}}\right)}{2 e^{-\tilde{\gamma} \tilde{\tau}}-1}$. Consequently, we get,

$$
\left\{\begin{array}{l}
x_{e}=\frac{1}{\tilde{\alpha}-1}\left[\tilde{\alpha} \beta^{-1}(\mu)-\tilde{\beta}^{-1}(\tilde{\mu})\right], \\
\tilde{x}_{e}=\frac{1}{\tilde{\alpha}-1}\left[\tilde{\beta}^{-1}(\tilde{\mu})-\beta^{-1}(\mu)\right], \\
\tilde{u}_{e}=\frac{\tilde{\delta}}{2 e^{-\tilde{\gamma} \tau}-1} \tilde{x}_{e} .
\end{array}\right.
$$

Now, we distinguish between the following two situations:

The case $\tilde{\alpha}=1$ : Here we notice that,

$$
\left\{\begin{array}{l}
x_{e}+\tilde{x}_{e}=\tilde{\beta}^{-1}(\tilde{\mu})=\beta^{-1}(\mu), \\
\tilde{u}_{e}=\frac{\tilde{\delta}}{2 e^{-\tilde{\gamma} \tilde{\tau}}-1} \tilde{x}_{e}
\end{array}\right.
$$


which is either an impossible case if the biological parameters are such that $\tilde{\beta}^{-1}(\tilde{\mu}) \neq \beta^{-1}(\mu)$, or, when $\tilde{\beta}^{-1}(\tilde{\mu})=\beta^{-1}(\mu)$, it corresponds to a continuum equilibrium point (the infinite possible values of $x_{e}$ and $\tilde{x}_{e}$ that satisfy the first equation in (18)). We want to avoid the latter continuum equilibrium points since that case has no concrete biological signification.

The case $\tilde{\alpha}>1$ or $0<\tilde{\alpha}<1$ : First, we focus on the case $0<\tilde{\alpha}<1$. We recall from earlier discussion that, biologically, $0<\tilde{\alpha}<1$ means that CSCs are less sensitive than ordinary cells to their shared environment composed by regulatory mitotic molecules (due to epigenetic mutations for instance, unhealthy cells no longer respond to inhibitory signals and continue to proliferate). More generally, $\tilde{\alpha}<1$ plays the role of a mitigating factor of the effect of regulatory molecules that attenuate the entrance frequency into proliferation. Now, from (17), we deduce that a sufficient condition for the existence of $\mathfrak{D}$ when $\tilde{\alpha}<1$, is given by: $\tilde{\alpha} \beta^{-1}(\mu)<\tilde{\beta}^{-1}(\tilde{\mu})<\beta^{-1}(\mu)$.

On the other hand, we observe that when $\tilde{\alpha}>1$, then, from (17), we deduce that a sufficient condition for the existence of $\mathfrak{D}$ is given by: $\beta^{-1}(\mu)<\tilde{\beta}^{-1}(\tilde{\mu})<$ $\tilde{\alpha} \beta^{-1}(\mu)$. We summarize the overall discussion in the following result:

Proposition 1. (i) For all $\tilde{\alpha}>0$, if the conditions

$$
\tilde{\delta}>\left[\frac{2 e^{-\tilde{\gamma} \tilde{\tau}}-1}{1-2 \tilde{K} e^{-\tilde{\gamma} \tilde{\tau}}}\right] \tilde{\beta}(0), \quad \text { and }, \quad \delta>\left[2 e^{-\gamma \tau}-1\right] \beta(0)
$$

are satisfied, then $(0,0,0)$ is the unique equilibrium point of the system (10). Note that in fact $(0,0,0)$ is always a steady state of the system (10).

(ii) For all $\tilde{\alpha}>0$, the condition

$$
\delta<\left[2 e^{-\gamma \tau}-1\right] \beta(0),
$$

is a necessary and sufficient conditions for the existence of the steady state, $\mathfrak{E}=\left(0,0, x_{e}\right)$, where $x_{e}>0$, for the system 10 . 
(iii) For all $\tilde{\alpha}>0$, if the conditions

$$
\tilde{\delta}>\left[\frac{2 e^{-\tilde{\gamma} \tilde{\tau}}-1}{1-2 \tilde{K} e^{-\tilde{\gamma} \tilde{\tau}}}\right] \tilde{\beta}(0), \quad \text { and }, \quad \delta<\left[2 e^{-\gamma \tau}-1\right] \beta(0)
$$

are satisfied, then $(0,0,0)$ and $\mathfrak{E}=\left(0,0, x_{e}\right)$ are the unique steady states of system 10 .

(iv) For all $\tilde{\alpha}>0$, the condition

$$
\tilde{\delta}<\left[\frac{2 e^{-\tilde{\gamma} \tilde{\tau}}-1}{1-2 \tilde{K} e^{-\tilde{\gamma} \tilde{\tau}}}\right] \tilde{\beta}(0)
$$
$\left(\tilde{x}_{e}, \tilde{u}_{e}, 0\right)$ where, $\tilde{x}_{e}>0$ and $\tilde{u}>0$, for the system 10 .

(v) For all $\tilde{\alpha}>0$, if the conditions

$$
\tilde{\delta}<\left[\frac{2 e^{-\tilde{\gamma} \tilde{\tau}}-1}{1-2 \tilde{K} e^{-\tilde{\gamma} \tilde{\tau}}}\right] \tilde{\beta}(0), \quad \text { and }, \quad \delta>\left[2 e^{-\gamma \tau}-1\right] \beta(0)
$$

are satisfied, then $(0,0,0)$ and $\left(\tilde{x}_{e}, \tilde{u}_{e}, 0\right)$ are the unique steady states of system (10).

(vi) For all $\tilde{\alpha}>0$, the conditions

$$
\tilde{\alpha} \neq 1, \quad \tilde{\delta}<\left[\frac{2 e^{-\tilde{\gamma} \tilde{\tau}}-1}{1-2 \tilde{K} e^{-\tilde{\gamma} \tilde{\tau}}}\right] \tilde{\beta}(0), \quad \text { and }, \quad \delta<\left[2 e^{-\gamma \tau}-1\right] \beta(0)
$$

are necessary, but not sufficient, for the existence of $\mathfrak{D}=\left(\tilde{x}_{e}, \tilde{u}_{e}, x_{e}\right)$.

(vii) We denote $\mu=\frac{\delta}{2 e^{-\gamma \tau}-1}$, and, $\tilde{\mu}=\frac{\tilde{\delta}\left(1-2 \tilde{K} e^{-\tilde{\gamma} \tilde{\tau}}\right)}{2 e^{-\tilde{\gamma} \tilde{\tau}}-1}$. If the conditions,

$$
\left\{\begin{array}{l}
0<\tilde{\alpha}<1, \quad \mu<\beta(0), \quad \tilde{\mu}<\tilde{\beta}(0) \\
\tilde{\alpha} \beta^{-1}(\mu)<\tilde{\beta}^{-1}(\tilde{\mu})<\beta^{-1}(\mu) \\
2 \tilde{K} e^{-\tilde{\gamma} \tilde{\tau}}<1<2 e^{-\tilde{\gamma} \tilde{\tau}}
\end{array}\right.
$$


or,

$$
\left\{\begin{array}{l}
\tilde{\alpha}>1, \quad \mu<\beta(0), \quad \tilde{\mu}<\tilde{\beta}(0), \\
\beta^{-1}(\mu)<\tilde{\beta}^{-1}(\tilde{\mu})<\tilde{\alpha} \beta^{-1}(\mu), \\
2 \tilde{K} e^{-\tilde{\gamma} \tilde{\tau}}<1<2 e^{-\tilde{\gamma} \tilde{\tau}}
\end{array}\right.
$$

are satisfied, then a unique strictly positive dormancy steady state $\mathfrak{D}=\left(\tilde{x}_{e}, \tilde{u}_{e}, x_{e}\right)$, exists and is given by (17).

Remark 2. (1) Obviously, uniqueness in Proposition 1-(vii) means the existence of a unique isolated strictly positive equilibrium point $\mathfrak{D}$, but the trivial steady state and the points $\mathfrak{E}=\left(0,0, x_{e}\right),\left(\tilde{x}_{e}, \tilde{u}_{e}, 0\right)$ are also steady states of system (10). (2) We can check that when the positive steady states exists, then

$$
y_{e}=\frac{1}{\gamma}\left(1-e^{-\gamma \tau}\right) \beta\left(x_{e}+\tilde{x}_{e}\right) x_{e}, \quad \text { and }, \quad \tilde{y}_{e}=\frac{1}{\tilde{\gamma}}\left(1-e^{-\tilde{\gamma} \tilde{\tau}}\right) \tilde{u}_{e}
$$

where $y_{e}$ (resp. $\tilde{y}_{e}$ ) is the positive steady state of the total density of proliferating healthy (resp. unhealthy) cells. (3) The third condition in (25)-26) expresses an interesting relationship between the fast-self renewing ability $\tilde{K}$, the apoptosis rate of malignant cancer cells $\tilde{\gamma}$, and their average cell-cycle duration $\tilde{\tau}$. We notice that even if $\tilde{K}$ is relatively important (and knowing that it is not easy to act on $\tilde{K}$ by drug infusions since its high value is due to FLT3 mutations) it is still possible to guarantee the existence of a dormancy state by increasing $\tilde{\tau} \tilde{\gamma}$. However, the increase must be moderate to not exceed the upper-bound $\tilde{\gamma} \tilde{\tau}<$ $\ln (2)$. (4) Finally, we notice that other cases can be discussed if biologically needed. For instance, adding the restriction, $2 \tilde{\beta}^{-1}(\tilde{\mu})<(1+\tilde{\alpha}) \beta^{-1}(\mu)$, to the conditions (25)-(26), ensures that $x_{e}>\tilde{x}_{e}$, which can reasonably be the expected situation of dormant tumors without forming clinically apparent cancers.

Now, we motivate our stability analysis through some preliminary numerical observations that highlight the rich dynamics of the model that we introduced in this work. In particular, we point out the different possible behaviors of the nonlinear differential-difference system (10) according to its associated initial conditions. The latter fact emphasizes the importance of determining mathe- 
matically an estimate of the region of attraction of each steady state of interest.

Example 1. Let us consider the following biological functions and parameters for cells in Category A (Cat. A) and Category B (Cat. B):

\begin{tabular}{ccccccc}
\hline Cat. A: & $\tau=1.11$ & $\delta=0.1$ & $\gamma=0.1$ & $\beta(m)=\frac{3}{1+m^{4}}$ & & \\
\hline Cat. B: & $\tilde{\tau}=0.9$ & $\tilde{\delta}=0.36$ & $\tilde{\gamma}=0.32$ & $\tilde{\beta}(m)=\frac{2}{1+m^{4}}$ & $\tilde{\alpha}=0.6$ & $\tilde{K}=0.54$ \\
\hline
\end{tabular}

For the considered set of parameters and functions, a unique dormancy steady state $\mathfrak{D}$ exists and is given by $\mathfrak{D}=\left(\tilde{x}_{e}, \tilde{u}_{e}, x_{e}\right)$, where $\tilde{x}_{e}=0.6573, \tilde{u}_{e}=$ 0.4737 and $x_{e}=1.5255$. This steady state is shown in Figure \& (a). However, the latter point is not the unique equilibrium point of the system. Indeed, the 0-equilibrium $(0,0,0)$, and the points: $\mathfrak{E}=(0,0,2.1826)$ and $(3.1998,2.3060,0)$, also exis ${ }^{13}$. When we select the constant initial conditions $\varphi_{x}(t)=\varphi_{\tilde{x}}(t)=2$, for all $t \in[-\tau, 0]$, and $\varphi_{\tilde{u}}(t)=1$, for all $t \in[-\tilde{\tau}, 0]$, we observe that the trajectories converge to $(3.1998,2.3060,0)$, as illustrated in Figure \&(b), where unhealthy cells survive (the attractive point seems to be stable), while the healthy cells vanish (converge to zero). By changing the initial condition of $\tilde{u}$, from the previous value to $\varphi_{\tilde{u}}(t)=0.1$, for all $t \in[-\tilde{\tau}, 0]$, we observe that the trajectories converge to $\mathfrak{E}$, as illustrated in Figure 4 - (c). Moreover, the steady states in Figures 4-(b)-(c) seem to be stable (each one has its region of attraction). Lyapunov theory offers strong tools to establish the regional stability properties of the steady states, provided that a suitable Lyapunov functional is found for the studied model. Now, let us modify the value of $\tilde{K}$ by increasing it to $\tilde{K}=0.6680$. It follows that $2 \tilde{K} e^{-\tilde{\gamma} \tilde{\tau}}-1=0.017$, which implies that the trajectories of the unhealthy compartment are unbounded (similarly to [1]). Numerical simulations in that case, for arbitrary initial conditions, are given in Figure 4 (d).

\footnotetext{
${ }^{13}$ One may notice the relationship that exists between the three different non-trivial steady states. In fact, the $x_{e}$-value in $\mathfrak{E}$ corresponds to the sum $x_{e}+\tilde{x}_{e}$ of the dormancy steady state $\mathfrak{D}$, while the $\tilde{x}_{e}$-value in the steady state $\left(\tilde{x}_{e}, \tilde{u}_{e}, 0\right)$ corresponds to the value $\frac{x_{e}+\tilde{\alpha} \tilde{x}_{e}}{\tilde{\alpha}}$, where $x_{e}$ and $\tilde{x}_{e}$ in the latter fraction are the corresponding values in the dormancy steady state $\mathfrak{D}$.
} 

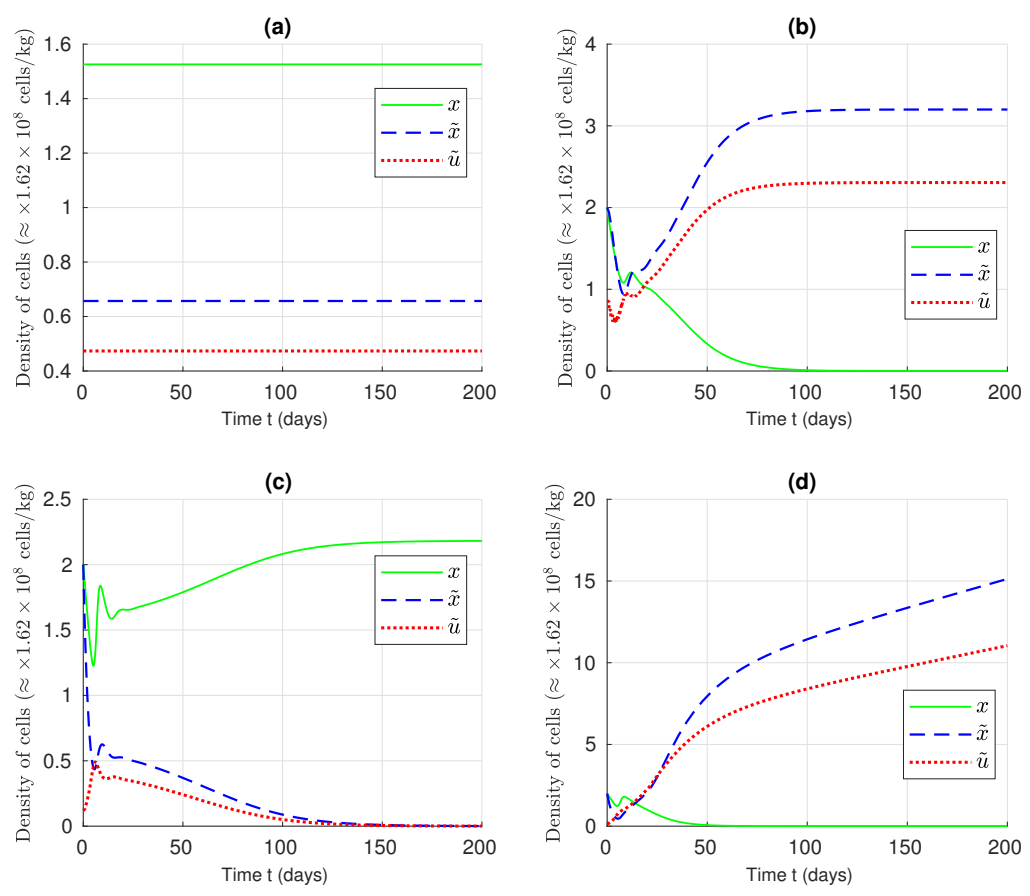

Figure 4: (a) Trajectories of the model whose parameters are given in Example 1, when they started on the Dormancy steady state $\mathfrak{D}$, where $\tilde{x}_{e}=0.6573, \tilde{u}_{e}=0.4737$ and $x_{e}=1.5255$. We mention that in this case, numerical simulations show that $\mathfrak{D}$ is unstable, i.e. for arbitrary small perturbation on the initial conditions, trajectories do not converge towards $\mathfrak{D}$. (b) Trajectories of the model whose parameters are given in Example 1 when they started from the initial conditions given by: $\varphi_{x}(t)=\varphi_{\tilde{x}}(t)=2$, for all $t \in[-\tau, 0]$, and $\varphi_{\tilde{u}}(t)=1$, for all $t \in[-\tilde{\tau}, 0]$. The steady states $\mathfrak{D}$ and $\mathfrak{E}$ both exist in this case, however, we notice that the trajectories rather converge to another equilibrium point, that seems stable, and which is given by $(3.1998,2.3060,0)$. (c) All the model parameters and the initial conditions are similar to (b), except the initial condition for $\tilde{u}$ which is no given by: $\varphi_{\tilde{u}}(t)=0.1$, for all $t \in[-\tilde{\tau}, 0]$. In this case, the trajectories converge to $\mathfrak{E}=(0,0,2.1826)$. (d) Now, we modify the value of $\tilde{K}$, that we incrase to 0.6680 , and we observe that for any initial conditions the trajectories $\tilde{x} \rightarrow+\infty$ and $\tilde{u} \rightarrow+\infty$, when $t$ goes to $+\infty$.

Example 2. Now, let us consider the following functions and parameters:

\begin{tabular}{lllllll}
\hline Cat. A: & $\tau=1.25$ & $\delta=0.2$ & $\gamma=0.2$ & $\beta(m)=\frac{1}{1+m^{2}}$ & & \\
\hline Cat. B: & $\tilde{\tau}=1.66$ & $\tilde{\delta}=0.1$ & $\tilde{\gamma}=0.2$ & $\tilde{\beta}(m)=\frac{1.2}{1+5 m^{4}}$ & $\tilde{\alpha}=0.4$ & $\tilde{K}=0.3$ \\
\hline
\end{tabular}

The steady states $(0,0,0), \mathfrak{E}=\left(0,0, x_{e}\right),\left(\tilde{x}_{e}, \tilde{u}_{e}, 0\right)$, and $\mathfrak{D}=\left(\tilde{x}_{e}, \tilde{u}_{e}, x_{e}\right)$, of the corresponding system, exist. If we select the constant initial conditions $\varphi_{x}(t)=1.55$, and $\varphi_{\tilde{x}}(t)=1$, for all $t \in[-\tau, 0]$, and $\varphi_{\tilde{u}}(t)=0.3$, for all $t \in$ 
470 (a), knowing that the dormancy steady state here is $\mathfrak{D}=(0.3445,0.0792,0.9926)$. For instance, we recall that in hematopoietic systems, oscillations are associated to many periodic diseases (e.g. cyclic neutropenia and some types of chronic leukemia). Now, let us consider random constant initial conditions and let us keep constant all the biological parameters, except the value of $\tilde{\alpha}$, that we consider as 0.2 , and then 0.6 in a second case. As shown in Figure 5-(b), we note that by increasing the value of $\tilde{\alpha}$ from 0.2 to 0.6 , the trajectories become stable. Thus, it appears that $\tilde{\alpha}$ may have, at least in this example, a stabilizing (or destabilizing) effect on the trajectories of the studied model.
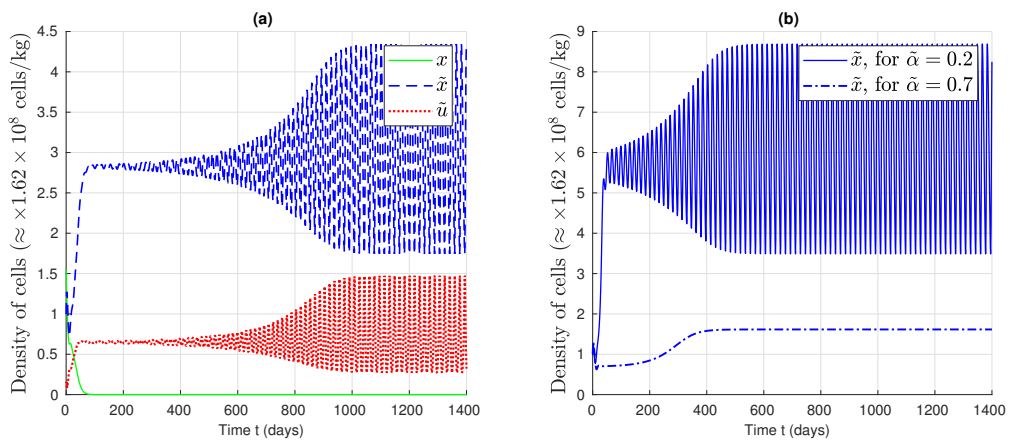

Figure 5: Trajectories of the model in Example 2 (a) Unstable (oscillatory) solutions corresponding to the constant initial conditions $\varphi_{x}(t)=1.55, \varphi_{\tilde{x}}(t)=1$, for all $t \in[-\tau, 0]$, $\varphi_{\tilde{u}}(t)=0.3$, for all $t \in[-\tilde{\tau}, 0]$. (b) Stabilizing effect of $\tilde{\alpha}$. In this illustration, all the parameters, as well as initial conditions, are identical, except the value of $\tilde{\alpha}$. In the first case, $\tilde{\alpha}=0.2$ : the trajectories are unstable. By increasing $\tilde{\alpha}$ to 0.7 the trajectories become stable.

Example 3. Finally, let us consider the following functions and parameters:

\begin{tabular}{ccccccc}
\hline Cat. A: & $\tau=1.25$ & $\delta=0.1$ & $\gamma=0.2$ & $\beta(m)=\frac{1}{1+m^{2}}$ & & \\
\hline Cat. B: & $\tilde{\tau}=0.7$ & $\tilde{\delta}=0.2$ & $\tilde{\gamma}=0.1$ & $\tilde{\beta}(m)=\frac{2}{1+2 m^{4}}$ & $\tilde{\alpha}=2$ & $\tilde{K}=0.5$ \\
\hline
\end{tabular}

The conditions of existence of $\mathfrak{D}=\left(\tilde{x}_{e}, \tilde{u}_{e}, x_{e}\right)$ are satisfied, and in this case we have: $\tilde{x}_{e}=0.6833, \tilde{u}_{e}=0.1580$ and $x_{e}=1.45599$. For the constant initial conditions $\varphi_{x}(t)=0.1$ and $\varphi_{\tilde{x}}(t)=1.5$, for all $t \in[-\tau, 0]$, and $\varphi_{\tilde{u}}(t)=1.5$ for all $t \in[-\tilde{\tau}, 0]$, it appears that $\mathfrak{D}$ is stable as illustrated in Figure 6 .

At this juncture, we deduce that the coupled system 10 under study has some interesting dynamical features. Firstly, we saw that the solutions of the 


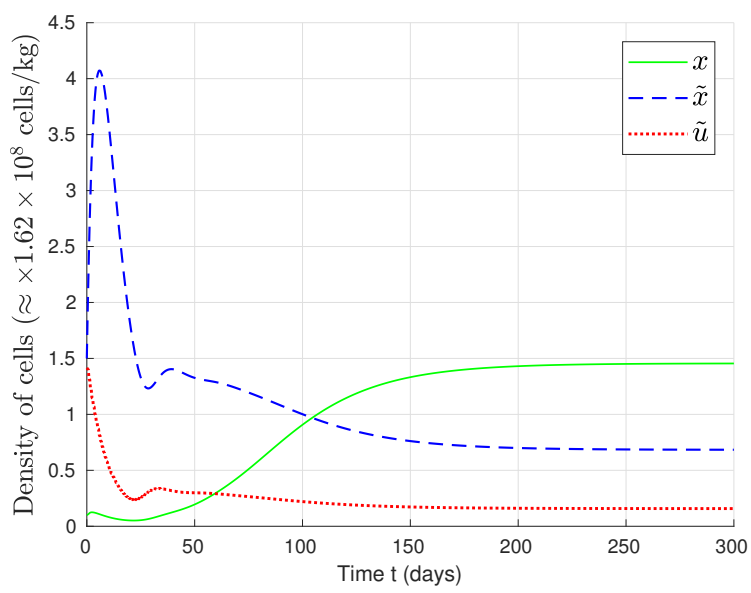

Figure 6: Trajectories of the model given in Example 3 Here the dormancy steady state $\mathfrak{D}$ exists and is given by: $\tilde{x}_{e}=0.6833, \tilde{u}_{e}=0.1580$ and $x_{e}=1.45599$. Convergence to the dormancy steady state $\mathfrak{D}$ (which seems stable) is obtained starting from the constant initial conditions: $\varphi_{x}(t)=0.1$ and $\varphi_{\tilde{x}}(t)=1.5$, for all $t \in[-\tau, 0]$, and $\varphi_{\tilde{u}}(t)=1.5$ for all $t \in[-\tilde{\tau}, 0]$.

coupled system can be bounded or unbounded (as in the non-coupled model [1]). In the former case, several steady states may exist and their values depend on the different biological parameters of the model. The existence of the steady states of interest $(\mathfrak{D}$ and $\mathfrak{E}$ ) are governed by some non-intuitive conditions on the biological parameters involved in the system (see Proposition 11). In addition, we saw that according to the initial conditions associated with the model trajectories, the bounded solutions may converge to one among several possible steady states, i.e. the stability of each steady state is regional (local). In the general case, the steady states of the system (10) are not always stable, but on the contrary, we noticed that oscillations may emerge, as in Example 2 . Our objective now is to determine exponential stability conditions for the steady states of interest (which are: all-cell extinction, $\mathfrak{E}$ and $\mathfrak{D}$ ).

\section{Stability analysis of the extinction of all the cells}

In this section, we perform a stability analysis of the 0-equilibrium of the system 10. From a biological standpoint, this is a case that we want to avoid, as 
discussed in the previous section (see the first point, Cell extinction), since it is synonymous of an excessive therapy that not only alters unhealthy populations, but also leads to the extinction of healthy cells in the coupled model.

Here we introduce the following functional:

$$
\begin{aligned}
\mathcal{W}\left(\tilde{x}_{t}, \tilde{u}_{t}, x_{t}\right)= & \tilde{x}(t)+x(t)+\psi_{1} \int_{t-\tilde{\tau}}^{t} e^{\rho_{1}^{*}(\ell-t)} \tilde{u}(\ell) d \ell \\
& +\psi_{2} \int_{t-\tau}^{t} e^{\rho_{2}^{*}(\ell-t)} \beta(x(\ell)+\tilde{x}(\ell)) x(\ell) d \ell
\end{aligned}
$$

505

Theorem 1. If the conditions

$$
\left(2 e^{-\gamma \tau}-1\right) \beta(0)<\delta, \quad 0<\psi^{*}, \quad \text { and }, \quad 2 \tilde{K} e^{-\tilde{\gamma} \tilde{\tau}}<1,
$$

are satisfied, then the trivial steady state of system 10 is globally exponentially stable with a decay rate larger than, or equal to, $\psi_{4}$ (defined in Appendix A).

Remark 3. (1) The conditions (29) exclude the existence of any steady state different from the trivial one. (2) We can interpret the cell extinction as a result of an excessive therapy that affects also healthy cells so that their apoptosis rate, $\gamma$, increases until becoming greater than the ratio $\frac{\ln (2)}{\tau}$, or, until the 
death rate and differentiation rate, i.e. $\delta$, becomes greater than $\left(2 e^{-\gamma \tau}-1\right) \beta(0)$

(which is a less demanding condition in comparison to $\left.\gamma>\frac{\ln (2)}{\tau}\right)$. (3) Arguing as in [1, 22], we can prove that the conditions (29) are also necessary for the exponential stability of the 0-equilibrium. (4) Finally, we deduce from Theorem 1 that all-cell extension results from uncorrelated conditions between the healthy and unhealthy compartments. Indeed, we note that the last two conditions in (29) relate to the unhealthy compartment, since only unhealthy parameters are involved. Moreover, these conditions are similar to those giving global asymptotic stability in [1] for a non-coupled model. The biological interpretation is that cell extension occurs if and only if both the healthy and unhealthy compartments are enable to regenerate themselves autonomously. In other words, it appears that the coupling has no effect on the stability of the 0-equilibrium since the conditions for total-cell eradication imply extinction of both the unhealthy and healthy compartments, as if they were separated (not coupled). This observation will not hold when we study dormancy (and non-zero steady states).

\section{Stability analysis of favourable steady states: $\mathfrak{D}$ (dormancy) and $\mathfrak{E}$ (CSCs eradication)}

Here we will emphasize on the dormancy steady state $\mathfrak{D}=\left(\tilde{x}_{e}, \tilde{u}_{e}, x_{e}\right)$, where all the components of the steady state are different from zero (i.e. $\tilde{x}_{e}>0, \tilde{u}_{e}>0$, $x_{e}>0$ ). In fact, we will highlight the case of dormancy $\mathfrak{D}$, since it is clearly the most general one. Indeed, from the analysis of $\mathfrak{D}$, it becomes possible to evaluate the regional stability properties of $\mathfrak{E}=\left(0,0, x_{e}\right)$ (which are partially investigated in [23], when $\tilde{\alpha}=1)$, and also of the steady state $\left(\tilde{x}_{e}, \tilde{u}_{e}, 0\right)$.

\subsection{A new representation of the system}

Now, we want to investigate the stability properties of $\mathfrak{D}$ when it exists. Thus, we assume that the conditions given in Proposition 1 (vii) are satisfied and we perform the classical changes of coordinates: $\tilde{X}=\tilde{x}-\tilde{x}_{e}, \tilde{U}=\tilde{u}-\tilde{u}_{e}$, 
and $X=x-x_{e}$. Therefore, from 100 , it follows that for all $t \geq 0$,

$$
\left\{\begin{array}{c}
\dot{\tilde{X}}(t)=-\left[\tilde{\delta}+\tilde{\beta}\left(X(t)+\tilde{\alpha} \tilde{X}(t)+x_{e}+\tilde{\alpha} \tilde{x}_{e}\right)\right]\left(\tilde{X}(t)+\tilde{x}_{e}\right) \\
+2(1-\tilde{K}) e^{-\tilde{\gamma} \tilde{\tau}}\left(\tilde{U}(t-\tilde{\tau})+\tilde{u}_{e}\right) \\
\tilde{U}(t)+\tilde{u}_{e}=\tilde{\beta}\left(X(t)+\tilde{\alpha} \tilde{X}(t)+x_{e}+\tilde{\alpha} \tilde{x}_{e}\right)\left(\tilde{X}(t)+\tilde{x}_{e}\right) \\
\quad+2 \tilde{K} e^{-\tilde{\gamma} \tilde{\tau}}\left(\tilde{U}(t-\tilde{\tau})+\tilde{u}_{e}\right) \\
\dot{X}(t)=-\left[\delta+\beta\left(X(t)+\tilde{X}(t)+x_{e}+\tilde{x}_{e}\right)\right]\left(X(t)+x_{e}\right) \\
+2 e^{-\gamma \tau} \beta\left(X(t-\tau)+\tilde{X}(t-\tau)+x_{e}+\tilde{x}_{e}\right)\left(X(t-\tau)+x_{e}\right) .
\end{array}\right.
$$

To ease the analysis of the above system, we rewrite it in a more convenient form. Observe that for all $\mathfrak{z}>-\mathfrak{e}, \mathfrak{e}>0$, where, $\mathfrak{z}=X+\tilde{X}$ and $\mathfrak{e}=x_{e}+\tilde{x}_{e}$, we have, with an abuse of notation,

$$
\beta(\mathfrak{z}+\mathfrak{e})=\beta(\mathfrak{e})+\theta \mathfrak{z}+R(\mathfrak{z}),
$$

where $\beta$ is the Hill-function defined in (1), $\theta=\beta^{\prime}(\mathfrak{e})$, and, $R(\mathfrak{z})=\int_{\mathfrak{e}}^{\mathfrak{e}+\mathfrak{z}}(\mathfrak{z}+\mathfrak{e}-$ $\ell) \beta^{(2)}(\ell) d \ell$. Next, for all $\tilde{\mathfrak{z}}>-\tilde{\mathfrak{e}}, \tilde{\mathfrak{e}}>0$, where, $\tilde{\mathfrak{z}}=X+\tilde{\alpha} \tilde{X}$, and, $\tilde{\mathfrak{e}}=x_{e}+\tilde{\alpha} \tilde{x}_{e}$, we get similarly to 31 ,

$$
\tilde{\beta}(\tilde{\mathfrak{z}}+\tilde{\mathfrak{e}})=\tilde{\beta}(\tilde{\mathfrak{e}})+\tilde{\theta} \tilde{\mathfrak{z}}+\tilde{R}(\tilde{\mathfrak{z}}),
$$

where, $\tilde{\theta}=\tilde{\beta}^{\prime}(\tilde{\mathfrak{e}})$, and, $\tilde{R}(\tilde{\mathfrak{z}})=\int_{\tilde{\mathfrak{e}}}^{\tilde{\mathfrak{e}}+\tilde{\mathfrak{z}}}(\tilde{\mathfrak{z}}+\tilde{\mathfrak{e}}-\ell) \tilde{\beta}^{(2)}(\ell) d \ell$. Therefore, using 31$)-(32)$, and by simplifying some terms using (11), we get the system,

$$
\left\{\begin{array}{l}
\dot{\tilde{X}}(t)=-\mathfrak{a}_{1} \tilde{X}(t)-\mathfrak{a}_{2} X(t)+\mathfrak{a}_{3} \tilde{U}(t-\tilde{\tau})+F(X(t), \tilde{X}(t)), \\
\tilde{U}(t)=\mathfrak{a}_{4} \tilde{X}(t)+\mathfrak{a}_{2} X(t)+\mathfrak{a}_{5} \tilde{U}(t-\tilde{\tau})-F(X(t), \tilde{X}(t)), \\
\dot{X}(t)=-\mathfrak{a}_{6} X(t)-\mathfrak{a}_{7} \tilde{X}(t)+\mathfrak{a}_{8} X(t-\tau)+\mathfrak{a}_{9} \tilde{X}(t-\tau)+G\left(X_{t}, \tilde{X}_{t}\right),
\end{array}\right.
$$

$$
\text { where, } \quad \begin{aligned}
F(X(t), \tilde{X}(t))= & \left.-\tilde{\theta}\left[\tilde{\alpha} \tilde{X}^{2}(t)\right)+X(t) \tilde{X}(t)\right] \\
& -\tilde{R}(X(t)+\tilde{\alpha} \tilde{X}(t))\left(\tilde{X}(t)+\tilde{x}_{e}\right),
\end{aligned}
$$




$$
\begin{aligned}
G\left(X_{t}, \tilde{X}_{t}\right)= & -\theta\left[X^{2}(t)+X(t) \tilde{X}(t)\right]-R(X(t)+\tilde{X}(t))\left(X(t)+x_{e}\right) \\
& +2 e^{-\gamma \tau} \theta\left[X^{2}(t-\tau)+X(t-\tau) \tilde{X}(t-\tau)\right] \\
& +2 e^{-\gamma \tau} R(X(t-\tau)+\tilde{X}(t-\tau))\left(X(t-\tau)+x_{e}\right),
\end{aligned}
$$

and where the constant parameters $\mathfrak{a}_{i}$ are given by:

$$
\left\{\begin{array}{l}
\mathfrak{a}_{1}=\tilde{\delta}+\tilde{\beta}\left(x_{e}+\tilde{\alpha} \tilde{x}_{e}\right)+\tilde{\alpha} \tilde{\theta} \tilde{x}_{e}, \quad \mathfrak{a}_{2}=\tilde{\theta} \tilde{x}_{e}, \quad \mathfrak{a}_{3}=2(1-\tilde{K}) e^{-\tilde{\gamma} \tilde{\tau}} \\
\mathfrak{a}_{4}=\tilde{\beta}\left(x_{e}+\tilde{\alpha} \tilde{x}_{e}\right)+\tilde{\alpha} \tilde{\theta} \tilde{x}_{e}, \mathfrak{a}_{5}=2 \tilde{K} e^{-\tilde{\gamma} \tilde{\tau}}, \mathfrak{a}_{6}=\delta+\beta\left(x_{e}+\tilde{x}_{e}\right)+\theta x_{e} \\
\mathfrak{a}_{7}=\theta x_{e}, \quad \mathfrak{a}_{8}=2 e^{-\gamma \tau}\left[\beta\left(x_{e}+\tilde{x}_{e}\right)+\theta x_{e}\right], \quad \mathfrak{a}_{9}=2 e^{-\gamma \tau} \theta x_{e} .
\end{array}\right.
$$

We notice that if the trajectories of 33 converge exponentially to the 0equilibrium, then the positive trajectories of the system (10) converge exponentially to $\mathfrak{D}$. Now, we are going to state and prove some sector conditions on the nonlinear terms $R$ and $\tilde{R}$. Then, we deduce some upper-bounds on the nonlinear terms $F$ and $G$. For that purpose, we prove in Appendix B through lengthy calculations that there exist strictly positive constants $\mathfrak{s}, \tilde{\mathfrak{s}}, \mathfrak{m}$ and $\tilde{\mathfrak{m}}$, satisfying:

$$
\begin{aligned}
|R(\mathfrak{z})| \leq \mathfrak{s}|\mathfrak{z}|, & \text { and }|\tilde{R}(\tilde{\mathfrak{z}})| \leq \tilde{\mathfrak{s}}|\tilde{\mathfrak{z}}| \\
|R(\mathfrak{z})| \leq \mathfrak{m} \mathfrak{z}^{2}, & \text { and }|\tilde{R}(\tilde{\mathfrak{z}})| \leq \tilde{\mathfrak{m}} \tilde{\mathfrak{z}}^{2}
\end{aligned}
$$

for all $\mathfrak{z}>-\mathfrak{e}(\mathfrak{z}$ and $\mathfrak{e}$ are defined before (31)), and for all $\tilde{\mathfrak{z}}>-\tilde{\mathfrak{e}}(\tilde{\mathfrak{z}}$ and $\tilde{\mathfrak{e}}$ are defined before (32). Moreover, using (37) and (38), we can determine strictly positive constants $\mathfrak{c}_{i}, i=\{1, \ldots, 6\}$, such that the following quadratic upper bounds hold true:

$$
\begin{gathered}
|F(X, \tilde{X})| \leq \mathfrak{c}_{1} Q(X)+\mathfrak{c}_{2} Q(\tilde{X}) \\
\left|G\left(X_{t}, \tilde{X}_{t}\right)\right| \leq \mathfrak{c}_{3} Q(X(t))+\mathfrak{c}_{4} Q(\tilde{X}(t))+\mathfrak{c}_{5} Q(X(t-\tau))+\mathfrak{c}_{6} Q(\tilde{X}(t-\tau)) .
\end{gathered}
$$

Remark 4. (1) The upper-bounds given in (37), (38), (39), and, 440, will not intervene when we determine the decay conditions and the decay rate of the 
solutions. However, their effect appears in the size of the basin of attraction that

approach) which relies on the analytic construction of a suitable Lyapunov-like functional, specific for the studied biological system. The latter approach allows us to provide more explicit decay conditions than the common LMI-type 
approaches. We point out that even if the second construction provides more conservative conditions than the LMI ones, they have the advantage of being more easily (biologically) understandable. It is to this end that, in the last section, we show how the decay conditions can be interpreted, in practice, according to the biological context of hematopoiesis and leukemia.

In summary, we determine throughout this section some exponential decay conditions (along with an estimate of the decay rate of the solutions and a region of attraction of the favourable steady states), via two complementary approaches: the descriptor method that provides local stability results for the general structure of the studied system, and, a suitable explicit Lyapunov-like construction that allows us to address the regional stability properties of the dormancy steady state. The latter decay conditions lend themselves more easily than the LMI ones to medical interpretations.

\subsection{Stability analysis using the descriptor method}

In this section, we consider as a first step only continuous solutions of the system in 33 and we study the linear approximation of the state $\operatorname{col}\{X, \tilde{X}\}$, that we denote $Z=\operatorname{col}\left\{Z_{1}, Z_{2}\right\}$. Then, by neglecting the nonlinear terms $F$ and $G$ in $(33)$, we rewrite the studied system in the following compact form:

$$
\left\{\begin{array}{l}
\dot{Z}(t)=B_{0} Z(t)+B_{1} Z(t-\tau)+B_{2} \tilde{U}(t-\tilde{\tau}), \\
\tilde{U}(t)=B_{3} Z(t)+B_{4} \tilde{U}(t-\tilde{\tau}),
\end{array}\right.
$$

for all $t \geq 0$, where $B_{i}$ are given by (we recall that $\mathfrak{a}_{i}$ are defined in $(36)$ ),

$$
\begin{aligned}
& B_{0}=-\left(\begin{array}{cc}
\mathfrak{a}_{6} & \mathfrak{a}_{7} \\
\mathfrak{a}_{2} & \mathfrak{a}_{1}
\end{array}\right), B_{1}=\left(\begin{array}{cc}
\mathfrak{a}_{8} & \mathfrak{a}_{9} \\
0 & 0
\end{array}\right), B_{2}=\left(\begin{array}{c}
0 \\
\mathfrak{a}_{3}
\end{array}\right), \\
& B_{3}=\left(\begin{array}{ll}
\mathfrak{a}_{2} & \mathfrak{a}_{4}
\end{array}\right), \text { and, } B_{4}=\mathfrak{a}_{5}=2 \tilde{K} e^{-\tilde{\gamma} \tilde{\tau}} .
\end{aligned}
$$

Next, we consider some symmetric positive definite matrices $P>0, S>0$, $J>0$, of appropriate dimension, together with a strictly positive constant $\tilde{\mathfrak{a}}$, 
and we verify that the derivative of the functional,

$$
\begin{aligned}
V\left(Z_{t}, \tilde{U}_{t}\right)= & Z(t)^{T} P Z(t)+\int_{t-\tau}^{t} Z^{T}(\ell) S Z(\ell) d \ell+ \\
& +\tilde{\mathfrak{a}} \int_{t-\tilde{\tau}}^{t} \tilde{U}^{2}(\ell) d \ell+\tau \int_{t-\tau}^{t}(\ell+\tau-t) \dot{Z}^{T}(\ell) J \dot{Z}(\ell) d \ell,
\end{aligned}
$$

along the trajectories of 41, is given by,

$$
\begin{aligned}
\dot{V}(t)= & Z^{T}(t)\left[P+P^{T}\right] \dot{Z}(t)+Z^{T}(t) S Z(t)-Z^{T}(t-\tau) S Z(t-\tau) \\
& -\tau \int_{t-\tau}^{t} \dot{Z}^{T}(\ell) J \dot{Z}(\ell) d \ell+\tau^{2} \dot{Z}^{T}(t) J \dot{Z}(t)+\tilde{\mathfrak{a}}\left(\tilde{U}^{2}(t)-\tilde{U}^{2}(t-\tilde{\tau})\right) .
\end{aligned}
$$

First, we notice that an upper-bound of $\dot{V}$ is given by,

$$
\begin{aligned}
\dot{V}(t) \leq & Z^{T}(t)\left[P+P^{T}\right] \dot{Z}(t)+Z^{T}(t) S Z(t)-Z^{T}(t-\tau) S Z(t-\tau) \\
& +\tau^{2} \dot{Z}^{T}(t) J \dot{Z}(t)-Z^{T}(t) J Z(t)+Z^{T}(t) J Z(t-\tau) \\
& +Z^{T}(t-\tau) J Z(t)-Z^{T}(t-\tau) J Z(t-\tau)+\tilde{\mathfrak{a}} \tilde{U}^{2}(t)-\tilde{\mathfrak{a}} \tilde{U}^{2}(t-\tilde{\tau}) \\
& +2\left[Z^{T}(t) \bar{P}^{T}+\dot{Z}^{T}(t) \overline{\bar{P}}^{T}\right] \underbrace{\left[B_{0} Z(t)+B_{1} Z(t-\tau)+B_{2} \tilde{U}(t-\tilde{\tau})-\dot{Z}(t)\right]}_{=0},
\end{aligned}
$$

which, in fact, directly follows from the Jensen's Inequality given by,

$$
\begin{aligned}
-\tau \int_{t-\tau}^{t} \dot{Z}^{T}(\ell) J \dot{Z}(\ell) d \ell & \leq-\int_{t-\tau}^{t} \dot{Z}^{T}(\ell) d \ell J \int_{t-\tau}^{t} \dot{Z}(\ell) d \ell \\
& =-[Z(t)-Z(t-\tau)]^{T} J[Z(t)-Z(t-\tau)]
\end{aligned}
$$

and where $\bar{P}$ and $\overline{\bar{P}}$ that appear in 44 are some free-weighting matrices of appropriate dimension. Then, it follows that,

$$
\dot{V}(t) \leq \eta^{T}(t) \Phi \eta(t)+\tilde{\mathfrak{a}} \tilde{U}^{2}(t)
$$

where $\eta$ is an augmented state defined by,

$$
\eta^{T}(t)=\left[\begin{array}{llll}
Z(t) & \dot{Z}(t) & Z(t-\tau) & \tilde{U}(t-\tilde{\tau})
\end{array}\right]
$$


and the matrix $\Phi$ is given by,

$$
\Phi=\left(\begin{array}{cccc}
S-J+\bar{p}^{T} B_{0}+B_{0}^{T} \bar{P} & P-\bar{P}^{T}+B_{0}^{T} \overline{\bar{P}} & J+\bar{P}^{T} B_{1} & \bar{P}^{T} B_{2} \\
* & \tau^{2} J-\overline{\bar{P}}^{T}-\overline{\bar{P}} & \overline{\bar{P}}^{T} B_{1} & \overline{\bar{P}}^{T} B_{2} \\
* & * & -S-J & 0 \\
* & * & * & -\tilde{\mathfrak{a}}
\end{array}\right) .
$$

Noticing that, $\tilde{U}(t)=\left[\begin{array}{cccc}B_{3} & 0 & 0 & B_{4}\end{array}\right] \eta(t)$, it follows that,

$\tilde{\mathfrak{a}} \tilde{U}^{2}(t)=\eta^{T}(t) E \eta(t)$, where, $E=\left[\begin{array}{llll}B_{3} & 0 & 0 & B_{4}\end{array}\right]^{T} \tilde{\mathfrak{a}}\left[\begin{array}{cccc}B_{3} & 0 & 0 & B_{4}\end{array}\right]$.

Therefore, by applying Schur complement, we conclude that $\dot{V}(t)<0$ is satisfied provided that the following LMI:

$$
\Psi=\left(\begin{array}{ccccc}
S-J+\bar{P}^{T} B_{0}+B_{0}^{T} \bar{P} & P-\bar{P}^{T}+B_{0}^{T} \overline{\bar{P}} & J+\bar{P}^{T} B_{1} & \bar{P}^{T} B_{2} & B_{3}^{T} \tilde{\mathfrak{a}} \\
* & \tau^{2} J-\overline{\bar{P}}^{T}-\overline{\bar{P}} & \overline{\bar{P}}^{T} B_{1} & \overline{\bar{P}}^{T} B_{2} & 0 \\
* & * & -S-J & 0 & 0 \\
* & * & * & -\tilde{\mathfrak{a}} & B_{4}^{T} \tilde{\mathfrak{a}} \\
* & * & * & * & -\tilde{\mathfrak{a}}
\end{array}\right)<0,
$$

holds. Next, by following arguments of [36] we deduce from $\Psi<0$ that the last block in 47 satisfies $\left(\begin{array}{cc}-\tilde{\mathfrak{a}} & B_{4}^{T} \tilde{\mathfrak{a}} \\ * & -\tilde{\mathfrak{a}}\end{array}\right)<0$. The latter implies by Schur complement that $-I+B_{4}^{T} B_{4}<0$. Hence, the eigenvalues of $B_{4}$ are inside the unit circle, i.e. the difference equation $\tilde{U}(t)=B_{4} \tilde{U}(t-\tilde{\tau})$ is stable for all $\tilde{\tau}>0$. The latter, together with $\dot{V}<0$, guarantees the asymptotic stability of the system (41). We mention that it is possible to extend the stability result to the nonlinear system (33), using the functional $V$ (i.e. providing some conditions on the nonlinear terms $F$ and $G$ as in [37, Section 3.11). However, since it seems actually difficult to interpret the LMI (47) as a combined targeted therapy for the studied biological system, we slightly modify our Lyapunov approach by 
designing, in the next section, a suitable specific LKF for the studied system that provides explicit (sufficient) stability conditions for the dormancy steady state of the nonlinear system (33). The functional that we are going to propose has some similarities with the functional $V$. Actually, in the next section, we are going to select some matrices $P, S$ and $J$, together with the constant $\tilde{\mathfrak{a}}$, involved in the above construction. Thus, we will determine analytically some upper-bounds on $\dot{V}$, through classical inequalities. Not surprisingly, the latter approach increases the conservatism of the sufficient stability condition in the LMI form (the LMI condition is given by (47)). That is the price of determining more biologically exploitable results (i.e. explicit exponential decay conditions with an estimate on the decay rate of the solution and a subset of the basin of attraction of the trivial steady state of the nonlinear system (33)).

\subsection{Obtaining Explicit Exponential Decay Conditions}

We focus on the coupled system using its representation in (33), with possibly piecewise continuous solutions. Firstly, let us introduce the quadratic function:

$$
\mathfrak{Q}(X, \tilde{X})=Q(X)+\lambda_{1} Q(\tilde{X}), \text { where, } Q(\ell)=\frac{1}{2} \ell^{2},
$$

and $\lambda_{1}=2$. This is equivalent to put $P=\operatorname{diag}\{1 / 2,1\}$ in $V$ of the previous section. Next, we consider the following operators,

$$
\begin{gathered}
\mathcal{Y}(\tilde{\varphi})=\int_{-\tilde{\tau}}^{0} e^{\rho_{1} \ell} Q(\tilde{\varphi}(\ell)) d \ell, \text { and } \\
\mathcal{S}(\varphi)=\int_{-\tau}^{0} e^{\rho_{2} \ell} Q(\varphi(\ell)) d \ell
\end{gathered}
$$

where, $\varphi \in \mathcal{C}([-\tau, 0], \mathbb{R}), \tilde{\varphi} \in \mathcal{C}([-\tilde{\tau}, 0], \mathbb{R})$, and $\rho_{1}, \rho_{2}$, are strictly positive constants that we determine later. In fact, observe that, compared to the integral terms in $V$ of the previous section, $\mathcal{S}$ and $\mathcal{Y}$ have exponential functions -in the integral terms- that make it possible to get a lower-bound on the exponential decay of the solutions. Next, in the quest for explicit decay conditions, we are

going to substitute $\dot{X}$ and $\dot{\tilde{X}}$ when computing the derivative of $\mathfrak{Q}$ (which is 
not the approach adopted in the descriptor method, where $\dot{X}$ and $\dot{\tilde{X}}$ were not replaced). Thus, the derivative of $\mathfrak{Q}$ along the trajectories of (33), satisfies

$$
\begin{aligned}
\dot{\mathfrak{Q}}(t)= & -2 \mathfrak{a}_{1} \lambda_{1} Q(\tilde{X}(t))-2 \mathfrak{a}_{6} Q(X(t))-\left(\mathfrak{a}_{2} \lambda_{1}+\mathfrak{a}_{7}\right) X(t) \tilde{X}(t) \\
& +\mathfrak{a}_{3} \lambda_{1} \tilde{X}(t) \tilde{U}(t-\tilde{\tau})+\mathfrak{a}_{8} X(t) X(t-\tau)+\mathfrak{a}_{9} X(t) \tilde{X}(t-\tau) \\
& +\lambda_{1} \tilde{X}(t) F(X(t), \tilde{X}(t))+X(t) G\left(X_{t}, \tilde{X}_{t}\right) .
\end{aligned}
$$

Notice that the derivative of $\mathcal{Y}\left(\tilde{U}_{t}\right)$, for almost all $t \geq 0$, is

$$
\dot{\mathcal{Y}}(t)=Q(\tilde{U}(t))-e^{-\rho_{1} \tilde{\tau}} Q(\tilde{U}(t-\tilde{\tau}))-\rho_{1} \mathcal{Y}\left(\tilde{U}_{t}\right) .
$$

Now, using the second equation in (33), we obtain

$$
\begin{aligned}
\dot{\mathcal{Y}}(t)= & -\rho_{1} \mathcal{Y}\left(\tilde{U}_{t}\right)+\mathfrak{a}_{4}^{2} Q(\tilde{X}(t))+\mathfrak{a}_{2}^{2} Q(X(t))-\left(e^{-\rho_{1} \tilde{\tau}}-\mathfrak{a}_{5}^{2}\right) Q(\tilde{U}(t-\tilde{\tau})) \\
& +\mathfrak{a}_{2} \mathfrak{a}_{4} X(t) \tilde{X}(t)+\mathfrak{a}_{2} \mathfrak{a}_{5} X(t) \tilde{U}(t-\tilde{\tau})+\mathfrak{a}_{4} \mathfrak{a}_{5} \tilde{X}(t) \tilde{U}(t-\tilde{\tau}) \\
& +Q(F(\tilde{X}(t), X(t)))-F(X(t), \tilde{X}(t))\left[\mathfrak{a}_{4} \tilde{X}(t)+\mathfrak{a}_{2} X(t)+\mathfrak{a}_{5} \tilde{U}(t-\tilde{\tau})\right],
\end{aligned}
$$

where the $\mathfrak{a}_{i}$ 's and $F$ are defined after (33). Similarly, we compute the derivatives of the functionals $\mathcal{S}\left(X_{t}\right)$ and $\mathcal{S}\left(\tilde{X}_{t}\right)$. By combining the previous intermediate results (i.e. $\dot{\mathfrak{Q}}, \dot{\mathcal{Y}}$ and $\dot{\mathcal{S}}$ ), we deduce that the time derivative of the functional,

$$
V^{\dagger}\left(X_{t}, \tilde{X}_{t}, \tilde{U}_{t}\right)=\mathfrak{Q}(X(t), \tilde{X}(t))+\lambda_{2} \mathcal{S}\left(X_{t}\right)+\lambda_{3} \mathcal{S}\left(\tilde{X}_{t}\right)+\lambda_{4} \mathcal{Y}\left(\tilde{U}_{t}\right),
$$

where $\lambda_{2}, \lambda_{3}$ and $\lambda_{4}$ are positive constants to be chosen later, along the trajectories of (33) is given, for almost all $t \geq 0$, by:

$$
\begin{aligned}
\dot{V}^{\dagger}(t)= & -\left[2 \lambda_{1} \mathfrak{a}_{1}-\lambda_{3}-\lambda_{4} \mathfrak{a}_{4}^{2}\right] Q(\tilde{X}(t))-\left[2 \mathfrak{a}_{6}-\lambda_{2}-\lambda_{4} \mathfrak{a}_{2}^{2}\right] Q(X(t)) \\
& -\rho_{2} \lambda_{3} \mathcal{S}\left(\tilde{X}_{t}\right)-\rho_{2} \lambda_{2} \mathcal{S}\left(X_{t}\right)-\rho_{1} \lambda_{4} \mathcal{Y}\left(\tilde{U}_{t}\right)-\lambda_{4}\left[e^{-\rho_{1} \tilde{\tau}}-\mathfrak{a}_{5}^{2}\right] Q(\tilde{U}(t-\tilde{\tau})) \\
& -\lambda_{2} e^{-\rho_{2} \tau} Q(X(t-\tau))-\lambda_{3} e^{-\rho_{2} \tau} Q(\tilde{X}(t-\tau))+\mathfrak{a}_{2} \mathfrak{a}_{5} \lambda_{4} X(t) \tilde{U}(t-\tilde{\tau}) \\
& -\left[\mathfrak{a}_{2} \lambda_{1}+\mathfrak{a}_{7}-\lambda_{4} \mathfrak{a}_{2} \mathfrak{a}_{4}\right] X(t) \tilde{X}(t)+\mathfrak{a}_{8} X(t) X(t-\tau)+\mathfrak{a}_{9} X(t) \tilde{X}(t-\tau) \\
& +\left[\mathfrak{a}_{3} \lambda_{1}+\mathfrak{a}_{4} \mathfrak{a}_{5} \lambda_{4}\right] \tilde{X}(t) \tilde{U}(t-\tilde{\tau})-\mathfrak{a}_{5} \lambda_{4} F(X(t), \tilde{X}(t)) \tilde{U}(t-\tilde{\tau}) \\
& +X(t) G\left(X_{t}, \tilde{X}_{t}\right)+\lambda_{4} Q(F(\tilde{X}(t), X(t)))-\lambda_{4} F(X(t), \tilde{X}(t))\left[\mathfrak{a}_{4} \tilde{X}(t)+\mathfrak{a}_{2} X(t)\right] .
\end{aligned}
$$


Next, we recall that for strictly positive constants, $\nu_{i}>0, i=1$ to 5 , (that we will choose later), we have the following inequalities: $|X \tilde{X}| \leq \frac{1}{\nu_{1}} Q(X)+\nu_{1} Q(\tilde{X})$, $|X(t) X(t-\tau)| \leq \frac{1}{\nu_{2}} Q(X(t))+\nu_{2} Q(X(t-\tau)),|X(t) \tilde{X}(t-\tau)| \leq \frac{1}{\nu_{3}} Q(X(t))+$ $\nu_{3} Q(\tilde{X}(t-\tau)),|\tilde{X}(t) \tilde{U}(t-\tilde{\tau})| \leq \frac{1}{\nu_{4}} Q(\tilde{X}(t))+\nu_{4} Q(\tilde{U}(t-\tilde{\tau})),|X(t) \tilde{U}(t-\tilde{\tau})| \leq$ $\frac{1}{\nu_{5}} Q(X(t))+\nu_{5} Q(\tilde{U}(t-\tilde{\tau}))$. Therefore, it follows that the derivative $\dot{V}^{\dagger}(t)$ satisfies, for almost all $t \geq 0$, the following inequality:

$$
\begin{aligned}
\dot{V}^{\dagger}(t) \leq & -\left[2 \lambda_{1} \mathfrak{a}_{1}-\mathfrak{b}_{1}\right] Q(\tilde{X}(t))-\left[2 \mathfrak{a}_{6}-\mathfrak{b}_{2}\right] Q(X(t))-\rho_{2} \lambda_{3} \mathcal{S}\left(\tilde{X}_{t}\right) \\
& -\rho_{2} \lambda_{2} \mathcal{S}\left(X_{t}\right)-\rho_{1} \lambda_{4} \mathcal{Y}\left(\tilde{U}_{t}\right)-\left[\lambda_{4} e^{-\rho_{1} \tilde{\tau}}-\mathfrak{b}_{3}\right] Q(\tilde{U}(t-\tilde{\tau})) \\
& -\left[\lambda_{2} e^{-\rho_{2} \tau}-\mathfrak{b}_{4}\right] Q(X(t-\tau))-\left[\lambda_{3} e^{-\rho_{2} \tau}-\mathfrak{b}_{5}\right] Q(\tilde{X}(t-\tau)) \\
& +\lambda_{4} Q(F(\tilde{X}(t), X(t)))-\mathfrak{a}_{5} \lambda_{4} F(X(t), \tilde{X}(t)) \tilde{U}(t-\tilde{\tau}) \\
& +X(t) G\left(X_{t}, \tilde{X}_{t}\right)-\lambda_{4} F(X(t), \tilde{X}(t))\left[\mathfrak{a}_{4} \tilde{X}(t)+\mathfrak{a}_{2} X(t)\right]
\end{aligned}
$$

where,

$$
\left\{\begin{array}{l}
\mathfrak{b}_{1}=\lambda_{3}+\lambda_{4} \mathfrak{a}_{4}^{2}+\nu_{1}\left|\mathfrak{a}_{2} \lambda_{1}+\mathfrak{a}_{7}-\lambda_{4} \mathfrak{a}_{2} \mathfrak{a}_{4}\right| \\
\mathfrak{b}_{2}=\lambda_{2}+\lambda_{4} \mathfrak{a}_{2}^{2}+\frac{\left|\mathfrak{a}_{2} \lambda_{1}+\mathfrak{a}_{7}-\lambda_{4} \mathfrak{a}_{2} \mathfrak{a}_{4}\right|}{\nu_{1}}+\frac{\left|\mathfrak{a}_{8}\right|}{\nu_{2}}+\frac{\left|\mathfrak{a}_{9}\right|}{\nu_{3}}+\frac{\left|\mathfrak{a}_{2} \mathfrak{a}_{5}\right| \lambda_{4}}{\nu_{5}} \\
\mathfrak{b}_{3}=\lambda_{4} \mathfrak{a}_{5}^{2}+\nu_{4}\left|\mathfrak{a}_{3} \lambda_{1}+\mathfrak{a}_{4} \mathfrak{a}_{5} \lambda_{4}\right|+\nu_{5} \lambda_{4}\left|\mathfrak{a}_{2} \mathfrak{a}_{5}\right| \\
\mathfrak{b}_{4}=\nu_{2}\left|\mathfrak{a}_{8}\right|, \quad \text { and, } \quad \mathfrak{b}_{5}=\nu_{3}\left|\mathfrak{a}_{9}\right| .
\end{array}\right.
$$

Now we are ready to determine decay conditions that ensure the regional exponential stability of the trivial steady state of the system (33). The terms where $F$ and $G$ are involved in (54) will be used only to determine a subset of the basin of attraction of the trivial steady state of the system (33).

Let us focus on the constant which is multiplied by $Q(\tilde{U}(t-\tilde{\tau}))$ in 54 . Using the inequality $\left|\mathfrak{a}_{3} \lambda_{1}+\mathfrak{a}_{4} \mathfrak{a}_{5} \lambda_{4}\right| \leq \lambda_{1}\left|\mathfrak{a}_{3}\right|+\lambda_{4}\left|\mathfrak{a}_{4} \mathfrak{a}_{5}\right|$, we notice that the inequality $\lambda_{4} e^{-\rho_{1} \tilde{\tau}}-\mathfrak{b}_{3}>0$ is verified if

$$
\lambda_{4}\left(e^{-\rho_{1} \tilde{\tau}}-\mathfrak{a}_{5}^{2}-\nu_{4}\left|\mathfrak{a}_{4} \mathfrak{a}_{5}\right|-\nu_{5}\left|\mathfrak{a}_{2} \mathfrak{a}_{5}\right|\right)-\nu_{4} \lambda_{1}\left|\mathfrak{a}_{3}\right|>0 .
$$

${ }_{620} \quad$ For later use, we set $\mathfrak{d}_{1} \triangleq \lambda_{4}\left(e^{-\rho_{1} \tilde{\tau}}-\mathfrak{a}_{5}^{2}-\nu_{4}\left|\mathfrak{a}_{4} \mathfrak{a}_{5}\right|-\nu_{5}\left|\mathfrak{a}_{2} \mathfrak{a}_{5}\right|\right)-\nu_{4} \lambda_{1}\left|\mathfrak{a}_{3}\right|$. 
We deduce that the first decay condition is given by:

$$
\mathfrak{a}_{5}^{2}+\nu_{4}\left|\mathfrak{a}_{4} \mathfrak{a}_{5}\right|+\nu_{5}\left|\mathfrak{a}_{2} \mathfrak{a}_{5}\right|<1
$$

Indeed, the previous condition is necessary to guarantee that 56 is satisfied. Now, let us select $\nu_{4}=\frac{1}{2}\left|\mathfrak{a}_{4}\right|^{-1}$, and $\nu_{5}=\frac{1}{2}\left|\mathfrak{a}_{2}\right|^{-1}$, for $\mathfrak{a}_{4} \neq 0$ and $\mathfrak{a}_{2} \neq 0$. Using the definitions of $\mathfrak{a}_{i}$ 's, $\nu_{4}$ and $\nu_{5}$, it follows that the first decay condition (57) is equivalent to

$$
\left(2 \tilde{K} e^{-\tilde{\gamma} \tilde{\tau}}\right)^{2}+2 \tilde{K} e^{-\tilde{\gamma} \tilde{\tau}}<1 .
$$

Remark 5. One notices that we have deliberately chosen $\nu_{4}=\frac{1}{2}\left|\mathfrak{a}_{4}\right|^{-1}$, and, $\nu_{5}=\frac{1}{2}\left|\mathfrak{a}_{2}\right|^{-1}$, and that these choices are not unique. Indeed, our objective here is to determine a sufficient decay condition that involves only the unhealthy parameters of the permanently dividing subpopulation (for instance, the subpopulation with FLT3-type mutations in AML) which are, $\tilde{K}$, $\tilde{\tau}$ and $\tilde{\gamma}$. For that purpose, we derive a decay condition involving only the parameter $\mathfrak{a}_{5}$. Therefore, $\nu_{4}$ and $\nu_{5}$ are used in order to compensate $\mathfrak{a}_{4}$ and $\mathfrak{a}_{2}$. A more general form is given by $\nu_{4}=\tilde{\nu}_{4}\left|\mathfrak{a}_{4}\right|^{-1}, \nu_{5}=\tilde{\nu}_{5}\left|\mathfrak{a}_{2}\right|^{-1}$, where $\tilde{\nu}_{4}>0$, and, $\tilde{\nu}_{5}>0$. In this case, the decay condition $(58)$ rewrites as, $\left(2 \tilde{K} e^{-\tilde{\gamma} \tilde{\tau}}\right)^{2}+2\left(\tilde{\nu}_{4}+\tilde{\nu}_{5}\right) \tilde{K} e^{-\tilde{\gamma} \tilde{\tau}}<1$.

Now, notice that a direct consequence of the inequality (58) is that for all $\rho_{1} \in\left(0, \frac{1}{\tilde{\tau}} \ln \left(\frac{5}{1+4\left[\mathfrak{a}_{5}^{2}+\mathfrak{a}_{5}\right]}\right)\right)$, we get $e^{-\rho_{1} \tilde{\tau}}-\left[\mathfrak{a}_{5}^{2}+\mathfrak{a}_{5}\right]>\frac{1-\left[\mathfrak{a}_{5}^{2}+\mathfrak{a}_{5}\right]}{5}>0$. Consequently, we deduce that $\mathfrak{d}_{1}$, which is defined right after (56), and which is now equal to: $\mathfrak{d}_{1}=\lambda_{4}\left(e^{-\rho_{1} \tilde{\tau}}-\left[\mathfrak{a}_{5}^{2}+\mathfrak{a}_{5}\right]\right)-\nu_{4} \lambda_{1}\left|\mathfrak{a}_{3}\right|$, satisfies the inequality, $\mathfrak{d}_{1}>0$, for all $\lambda_{4}=\frac{\tilde{\lambda}_{4} \lambda_{1} \nu_{4}\left|\mathfrak{a}_{3}\right|}{e^{-\rho_{1} \tilde{\tau}}-\left[\mathfrak{a}_{5}^{2}+\mathfrak{a}_{5}\right]}>0$, where $\tilde{\lambda}_{4}>1$. Next, using the inequality,

$$
|F(X(t), \tilde{X}(t)) \tilde{U}(t-\tilde{\tau})| \leq \frac{2\left|\mathfrak{a}_{5}\right| \lambda_{4}}{\mathfrak{d}_{1}} Q(F(X(t), \tilde{X}(t)))+\frac{\mathfrak{d}_{1}}{2\left|\mathfrak{a}_{5}\right| \lambda_{4}} Q(\tilde{U}(t-\tilde{\tau}),
$$


it follows from (54) that,

$$
\begin{aligned}
\dot{V}^{\dagger}(t) \leq & -\left[2 \lambda_{1} \mathfrak{a}_{1}-\mathfrak{b}_{1}\right] Q(\tilde{X}(t))-\left[2 \mathfrak{a}_{6}-\mathfrak{b}_{2}\right] Q(X(t))-\frac{\mathfrak{d}_{1}}{2} Q(\tilde{U}(t-\tilde{\tau})) \\
& -\rho_{2} \lambda_{2} \mathcal{S}\left(X_{t}\right)-\rho_{2} \lambda_{3} \mathcal{S}\left(\tilde{X}_{t}\right)-\left[\lambda_{2} e^{-\rho_{2} \tau}-\mathfrak{b}_{4}\right] Q(X(t-\tau)) \\
& -\left[\lambda_{3} e^{-\rho_{2} \tau}-\mathfrak{b}_{5}\right] Q(\tilde{X}(t-\tau))-\rho_{1} \lambda_{4} \mathcal{Y}\left(\tilde{U}_{t}\right)+H\left(X_{t}, \tilde{X}_{t}\right),
\end{aligned}
$$

where,

$$
\begin{aligned}
H\left(X_{t}, \tilde{X}_{t}\right)= & \left(\lambda_{4}+\frac{2\left(\mathfrak{a}_{5} \lambda_{4}\right)^{2}}{\mathfrak{d}_{1}}\right) Q(F(X(t), \tilde{X}(t)))+X(t) G\left(X_{t}, \tilde{X}_{t}\right) \\
& -\lambda_{4} F(X(t), \tilde{X}(t))\left[\mathfrak{a}_{4} \tilde{X}(t)+\mathfrak{a}_{2} X(t)\right] .
\end{aligned}
$$

Arguing similarly, we select $\nu_{2}$ and $\nu_{3}$ that compensate the terms $\mathfrak{a}_{8}$ and $\mathfrak{a}_{9}$ (for $\left|\mathfrak{a}_{8}\right| \neq 0$, and $\left|\mathfrak{a}_{9}\right| \neq 0$ ). For instance, we can consider $\nu_{2}=\frac{1}{6\left|\mathfrak{a}_{8}\right|}$ and $\nu_{3}=\frac{1}{6\left|\mathfrak{a}_{9}\right|}$. Then, we put, for instance, $\lambda_{2}=\lambda_{3}=\frac{1}{3}$. We notice that our choices of $\nu_{2}$ and $\nu_{3}$ in this case are equivalent to $\mathfrak{b}_{4}=\mathfrak{b}_{5}=\frac{1}{6}$, and it follows that for all $\rho_{2} \in\left(0, \frac{1}{\tau} \ln \left(\frac{\lambda_{2}}{\mathfrak{b}_{4}}\right)\right)$, we obtain in this case $e^{-\rho_{2} \tau}>\frac{2}{3}$. Thus, we end up with 14

$$
\begin{aligned}
& \mathfrak{d}_{2} \triangleq \lambda_{2} e^{-\rho_{2} \tau}-\mathfrak{b}_{4}=\frac{1}{3}\left(e^{-\rho_{2} \tau}-\frac{1}{2}\right)>\frac{1}{18} \\
& \mathfrak{d}_{3} \triangleq \lambda_{3} e^{-\rho_{2} \tau}-\mathfrak{b}_{5}=\frac{1}{3}\left(e^{-\rho_{2} \tau}-\frac{1}{2}\right)>\frac{1}{18}
\end{aligned}
$$

Finally, by selecting $\nu_{1}=\lambda_{1}=2$, all the setting parameters involved in the functional $V^{\dagger}$ are now chosen. We conclude that if the decay conditions $\mathfrak{d}_{4} \triangleq 2 \lambda_{1} \mathfrak{a}_{1}-\mathfrak{b}_{1}>0$, and $\mathfrak{d}_{5} \triangleq 2 \mathfrak{a}_{6}-\mathfrak{b}_{2}>0$, are satisfied, then (59) satisfies for almost all $t \geq 0$,

$$
\begin{aligned}
\dot{V}^{\dagger}(t) \leq & -3 \overline{\mathfrak{d}} V^{\dagger}\left(X_{t}, \tilde{X}_{t}, \tilde{U}_{t}\right)-\frac{\mathfrak{d}_{4}}{2} Q(\tilde{X}(t))-\frac{\mathfrak{d}_{5}}{2} Q(X(t))-\frac{\mathfrak{d}_{1}}{2} Q(\tilde{U}(t-\tilde{\tau})) \\
& -\mathfrak{d}_{2} Q(X(t-\tau))-\mathfrak{d}_{3} Q(\tilde{X}(t-\tau))+H\left(X_{t}, \tilde{X}_{t}\right)
\end{aligned}
$$

\footnotetext{
${ }^{14}$ Similarly to $\nu_{4}$ and $\nu_{5}$ in Remark 5 the choices of $\nu_{2}$ and $\nu_{3}$ are not unique (and, similarly, those of $\lambda_{2}$ and $\lambda_{3}$ either). In Example 4 we are going to use different numerical values that also satisfy $\mathfrak{d}_{2}>0$ and $\mathfrak{d}_{3}>0$.
} 
where $\overline{\mathfrak{d}}=\frac{1}{3} \min \left\{\frac{\mathfrak{d}_{4}}{2 \lambda_{1}}, \frac{\mathfrak{d}_{5}}{2}, \rho_{1}, \rho_{2}\right\}$. Next, in Appendix C we focus on the nonlinear function $H$, defined right after $(59)$, in order to define a subset of the basin of attraction of the trivial steady state of system (33). By following the arguments given in Appendix C, we prove that in a well-defined region (defined in terms of the initial conditions) we get:

$$
\dot{V}^{\dagger}(t) \leq-2 \overline{\mathfrak{d}} V^{\dagger}\left(X_{t}, \tilde{X}_{t}, \tilde{U}_{t}\right), \text { for almost all } t \geq 0
$$

We integrate this inequality and we obtain for all $t \geq 0$,

$$
V^{\dagger}\left(X_{t}, \tilde{X}_{t}, \tilde{U}_{t}\right) \leq e^{-2 \overline{\mathfrak{d}} t} V^{\dagger}\left(\varphi_{X_{t}}, \varphi_{\tilde{X}_{t}}, \varphi_{\tilde{U}_{t}}\right)
$$

Consequently, we get for all $t \geq 0, X^{2}(t)+\lambda_{1} \tilde{X}^{2}(t) \leq 2 e^{-2 \bar{d} t} V^{\dagger}\left(\varphi_{X}, \varphi_{\tilde{X}}, \varphi_{\tilde{U}}\right)$. We conclude that the trajectories $X(t)$ and $\tilde{X}(t)$ converge exponentially to the trivial steady state of the shifted system, with a decay rate larger than, or equal to, $\overline{\mathfrak{d}}$. By classical arguments, we observe from the second equation in 33 that, ${ }_{635}$ since $2 \tilde{K} e^{-\tilde{\gamma} \tilde{\tau}}<1, \tilde{U}(t)$ converges exponentially to zero when $X(t)$ and $\tilde{X}(t)$ converge exponentially to the zero.

To summarize, we considered that $\mathfrak{D}$ (or $\mathfrak{E}$ ) exists and we rewrote the studied system 10 in the form 33 . Next, we proved that if the decay conditions $\left((58), \mathfrak{d}_{4}>0, \mathfrak{d}_{5}>0\right)$ are satisfied, then the trajectories of $(33)$ associated with initial conditions belonging to the set $\mathcal{B}$, converge exponentially to 0 -equilibrium of the shifted system $(33)$, with a decay rate larger than, or equal to, $\overline{\mathfrak{d}}$. By explicitly rewriting the decay conditions, we summarize our findings in Section 5 as follows:

Theorem 2. (A) Assume that $\mathfrak{D}$ (resp. $\mathfrak{E}$ ) exists, then consider the shifted system (33), such that its trivial steady state corresponds to $\mathfrak{D}$ (resp. $\mathfrak{E}$ ) of 10. If there exist matrices $P, S, J, \bar{P}$ and $\overline{\bar{P}}$, of appropriate dimension, and a positive constant $\tilde{\mathfrak{a}}$, that satisfy the LMI (47), then the trivial steady state of the shifted system (33), which is $\mathfrak{D}$ (resp. $\mathfrak{E}$ ) of 10 , is locally asymptotically stable. 
(B) Assume that system (10) admits a positive steady state $\mathfrak{D}$ (i.e. 25) or 26) in Proposition 1 (vii) hold). If

$$
\begin{aligned}
& \text { i) }\left(2 \tilde{K} e^{-\tilde{\gamma} \tilde{\tau}}\right)^{2}+2 \tilde{K} e^{-\tilde{\gamma} \tilde{\tau}}<1, \\
& \text { ii) } \frac{\mathfrak{b}_{1}}{4}-\tilde{\alpha} \tilde{\theta} \tilde{x}_{e}<\tilde{\beta}\left(x_{e}+\tilde{\alpha} \tilde{x}_{e}\right)+\tilde{\delta}, \\
& \text { iii) } \frac{\mathfrak{b}_{2}}{2}-\theta x_{e}<\beta\left(x_{e}+\tilde{x}_{e}\right)+\delta,
\end{aligned}
$$

are satisfied, ensuring also that $\mathfrak{d}_{2}>0$ and $\mathfrak{d}_{3}>0$, then $\mathfrak{D}$ is regionally exponentially stable with a decay rate larger than, or equal to, $\overline{\mathfrak{d}}$, and with basin of attraction defined by:

$$
\begin{array}{r}
\mathcal{B}^{\dagger}=\left\{\varphi_{x} \in \mathcal{C}\left([-\tau, 0], \mathbb{R}^{+}\right), \varphi_{\tilde{x}} \in \mathcal{C}\left([-\tau, 0], \mathbb{R}^{+}\right), \varphi_{\tilde{u}} \in \mathcal{C}\left([-\tilde{\tau}, 0], \mathbb{R}^{+}\right) \mid\right. \\
\left.V^{\dagger}\left(\varphi_{x}-x_{e}, \varphi_{\tilde{x}}-\tilde{x}_{e}, \varphi_{\tilde{u}}-\tilde{u}_{e}\right)<\bar{V}^{\dagger}\right\} .
\end{array}
$$

(C) Assume that $\mathfrak{E}$ exists (Proposition 1-(ii)), and consider that $\tilde{x}_{e}=0$ in (64).

If the conditions (64) are satisfied (for $\tilde{x}_{e}=0$ ), then $\mathfrak{E}$ of 10 is regionally exponentially stable with a decay rate $\mathfrak{d}$ and basin of attraction defined by (65), where we consider now that $\tilde{x}_{e}=\tilde{u}_{e}=0$ in 65 .

Example 4. In this example, we assume that $\tilde{\alpha}=5$. For the unhealthy compartment, we consider the parameters given in Table 1, while for the healthy case we consider the parameters of Table 2.

We want to investigate the stability properties of the dormancy steady state: $\mathfrak{D}=\left(\tilde{x}_{e}, \tilde{u}_{e}, x_{e}\right)$, where, $\tilde{x}_{e}=0.0217, \tilde{u}_{e}=0.0593$, and $x_{e}=0.2535$. Obviously, if the decay conditions (64) are satisfied, then the LMI (47) admits a solution.

\begin{tabular}{|c|c|c|c|c||c|c|}
\hline$\tilde{\delta}$ & $\tilde{\gamma}$ & $\tilde{\tau}$ & $\tilde{\beta}(m)$ & $\tilde{K}$ & $\tilde{u}_{e}$ & $\tilde{x}_{e}$ \\
\hline 0.928 & 0.4 & 1 & $\frac{2.78}{1+3 m^{2}}$ & 0.2 & 0.05938567 & 0.02179864 \\
\hline
\end{tabular}

Table 1: Parameters of the unhealthy compartment, and the values of $\tilde{x}_{e}$ and $\tilde{u}_{e}$.

\begin{tabular}{|c|c|c|c||c|}
\hline$\delta$ & $\gamma$ & $\tau$ & $\beta(m)$ & $x_{e}$ \\
\hline 0.168 & 0.001 & 0.12 & $\frac{0.219}{1+4 m^{2}}$ & 0.25354595 \\
\hline
\end{tabular}

Table 2: Parameters of the healthy hematopoetic stem cell compartment, and the value of $x_{e}$. 
We check that the decay conditions (64) are verified:

$$
\begin{aligned}
& \text { (i) } 1-2 \tilde{K} e^{-\tilde{\gamma} \tilde{\tau}}-\left(2 \tilde{K} e^{-\tilde{\gamma} \tilde{\tau}}\right)^{2}=0.659979347>0, \\
& \text { (ii) } \tilde{\beta}\left(x_{e}+\tilde{\alpha} \tilde{x}_{e}\right)+\tilde{\delta}-\left(\frac{\mathfrak{b}_{1}}{4}-\tilde{\alpha} \tilde{\theta} \tilde{x}_{e}\right)=0.987350196>0, \\
& \text { (iii) } \beta\left(x_{e}+\tilde{x}_{e}\right)+\delta-\left(\frac{\mathfrak{b}_{2}}{2}-\theta x_{e}\right)=0.000149333>0,
\end{aligned}
$$

where we consider: $\lambda_{1}=2, \lambda_{2}=\lambda_{3}=0.261780, \lambda_{4}=2.205796, \tilde{\lambda}_{4}=$ $2, \quad \nu_{1}=2, \quad \nu_{2}=\frac{1}{4\left|\mathfrak{a}_{8}\right|}=1.301858, \nu_{3}=\frac{1}{4\left|\mathfrak{a}_{9}\right|}=1.736024, \quad \nu_{4}=\frac{1}{2\left|\mathfrak{a}_{4}\right|}=$ $0.302151, \nu_{5}=\frac{1}{2\left|\mathfrak{a}_{2}\right|}=7.374022, \rho_{1}=\frac{1}{10 \tilde{\tau}} \ln \left(\frac{5}{1+4\left(\mathfrak{a}_{5}^{2}+\mathfrak{a}_{5}\right)}\right)=0.075074$ and $\rho_{2}=\frac{1}{10 \tau} \ln \left(\frac{\lambda_{2}}{\mathfrak{b}_{4}}\right)=0.038369$. For these numerical values, we check that $\mathfrak{d}_{2}=$ $\mathfrak{d}_{3}=0.010577>0$. Therefore, according to Theorem 2, the dormancy steady state, $\mathfrak{D}=(0.0217,0.0593,0.2535)$, is regionally exponentially stable, as illustrated in Figure 4. This example will be revisited in the next section, in the practical situation of therapeutic strategies.

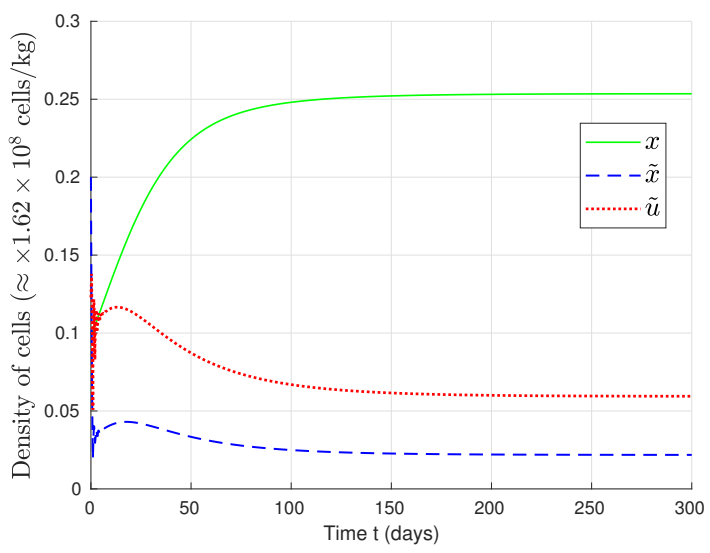

Figure 7: Trajectories of the system of the numerical example 4 (Tables 1.2. In this case, the dormancy steady state $\mathfrak{D}$ exists, such that $\tilde{x}_{e}=0.0217, \tilde{u}_{e}=0.0593$. The sufficient local stability conditions given in Theorem 2 (B) are satisfied, as shown in 66), and the trajectories of the system converge exponentially to $\mathfrak{D}$. 


\section{Concluding comments on the findings and possible therapeutic strategies oriented towards cancer dormancy}

A first remark is that CSC dormancy probably results from complex rela-

665 that are difficult to elicit, let alone to be understood. This observation concerns the stability properties (decay conditions in Theorem 2), but also the conditions of existence of dormancy (Proposition 1 1 (vii)), along with the role of the sensitivity parameter $\tilde{\alpha}$. This should lead us to develop further the mathematical 
steady states of interest are a type of red lines, that must not be crossed when elaborating a treatment strategy.

On the other hand, when we focus on the stability conditions, wondering how therapeutic actions can make the biological system go into the direction of the decay conditions (64), we realize that the respective decay conditions of $\mathfrak{D}$ and $\mathfrak{E}$ are substantially similar. More precisely, our sufficient stability conditions suggest that the biological parameters that can be targeted in order to satisfy (64), in either of the two states $\mathfrak{D}$ or $\mathfrak{E}$, are similar (but not identical). In this sense, we can state that a common therapeutic strategy for $\mathfrak{D}$ and $\mathfrak{E}$ can be proposed. So, in light of the existing therapies and recent clinical trials that highlight novel effective molecules as potential drugs in AML, we briefly discuss how a combined therapy - mostly composed of targeted therapies and standard chemotherapy - may satisfy the theoretical conditions (64).

First, we observe that the condition (B-i) in Theorem 2 provides a restriction on the dynamics of over-proliferating cells, since $\tilde{K}, \tilde{\gamma}$ and $\tilde{\tau}$ are involved. Satisfying the previous condition relies in increasing the product $\tilde{\gamma} \tilde{\tau}$, and decreasing $\tilde{K}$. Increasing $\tilde{\gamma} \tilde{\tau}$ means that we extend the average duration of the cell cycle $\tilde{\tau}$ and/or increase the apoptosis rate $\tilde{\gamma}$ in the population of unhealthy cells. Leukemic cells may be targeted by drugs such as quizartinib (AC220 94]) or erlotinib [54] to increase $\tilde{\tau}$, while cytosine arabinoside can be used to increase the apoptosis rate $\tilde{\gamma}$. Moreover, quizartinib can be used to decrease the fast selfrenewal rate $\tilde{K}$. In fact, $\tilde{K}$ is expected to be the hardest parameters to modify in practice, due to preexisting mutations in epigenetic control genes (DNMT3A, TET2). However, new FLT3 inhibitors, such as midostaurin 15 , have achieved good performance (see the recent quantitative results provided in [85]) and are now approved for use along with chemotherapy to target leukemic cells in AML.

Next, in the conditions (B-ii) and (B-iii) of Theorem 2, the targets can be the parameters $\delta$ and $\tilde{\delta}$ (mainly $\tilde{\delta}$, since it is the unhealthy parameter) that

\footnotetext{
${ }^{15}$ Midostaurin is a multi-targeted protein kinase inhibitor, which can be active against oncogenic CD135 (FMS-like tyrosine kinase 3 receptor, FLT3). 25 85
} 
$\tilde{\delta}$ includes the death rate and the differentiation rate of unhealthy resting cells. In practice, increasing $\tilde{\delta}$ means that we should increase the differentiation rates, which can be achieved in the case of leukemia by infusing dasatinib [54, that targets most of the tyrosine kinases including the c-KIT gene. In fact, it was not effective in the specific case of AML. However, this therapeutic option has been relaunched recently after successful clinical trials, where dihydroorotate dehydrogenase (DHODH) inhibitors restored differentiation of leukemic cells in AML 86. Finally, increasing $\beta(0)$ and $\tilde{\beta}(0)$ can be performed by using G-CSF molecules [34. These are the main common targets shared by $\mathfrak{D}$ and $\mathfrak{E}$.

\section{2) Constraints and spillover risks of CSCs eradication: Increasing}

the parameters $\tilde{\delta}, \tilde{\gamma}$ and $\tilde{\tau}$ (using some of the previously mentioned molecules or their equivalent), promotes the existence of the state $\mathfrak{E}$, together with its stability. However, it may exclude the steady state $\mathfrak{D}$, by violating its conditions of existence. Furthermore, an excessive therapy that affects also healthy cells leads, theoretically, to the extinction of all the cells (Theorem 1). At the other extreme, insufficient drug dose might not successfully stop CSCs from overproliferation (when $2 \tilde{K} e^{-\tilde{\gamma} \tilde{\tau}}>1$ ). The overproliferating behavior may be worsened by CSC resistance to drugs. Thus, dormancy $\mathfrak{D}$ appears as a delicate intermediate equilibrium between the cancer progression and CSC eradication.

3) Specific constraints related to dormancy: In the common strategy that aims to satisfy the condition (64), we noticed that drugs have to increase the product $\tilde{\gamma} \tilde{\tau}$. On the other hand, we recall from Proposition 1 (vii) that the condition $1<2 e^{-\tilde{\gamma} \tilde{\tau}}$ is necessary for the existence of $\mathfrak{D}$. Thus, the therapy action in this case has to take into account this supplementary condition. We infer from this remark that the probability to achieve the dormancy steady state $\mathfrak{D}$ by using the classical strategies that consist in giving the maximum tolerated dose of drugs during the treatment period [28], is therefore very low. Indeed, since a high dose is expected to yield $1>2 e^{-\tilde{\gamma} \tilde{\tau}}$, the condition of existence of 
in Proposition 1 show that the existence of $\mathfrak{D}$ is more difficult to achieve than the existence of $\mathfrak{E}$. However, we suggest that infusing G-CSF molecules appears to favour the existence of a dormancy steady state, since increasing (relatively) $\beta(0)$ seems to go in the right direction in order to satisfy both the existence and chosen an intuitive set of parameters that matches specific dysfunctions in overproliferating malignant hemopathies (particularly the condition $2 \tilde{K} e^{-\tilde{\gamma} \tilde{\tau}}>1$ ).

\begin{tabular}{|c|c|c|c|c|c|}
\hline$\tilde{\delta}$ & $\tilde{\gamma}$ & $\tilde{\tau}$ & $\tilde{\beta}(m)$ & $\tilde{K}$ & $\tilde{\alpha}$ \\
\hline 0.25 & 0.1 & 0.2 & $\frac{2.78}{1+m^{3}}$ & 0.55 & 0.8 \\
\hline
\end{tabular}

Table 3: The set of initial (i.e. before treatment) parameters of the unhealthy compartment.

On the other hand, we assume that the parameters of the healthy compartment are those given in Example 4, and we consider that the therapy to be administrated has a negligible effect on ordinary cells.

In medical practice, usually the hematopoietic system is targeted through 
chemotherapy or targeted therapy (a combination of two or three drugs), sometimes infused along with a complementary treatment. All these drugs have in fact molecular targets (e.g. dasatinib targets BCR/Abl, Src, ephrin receptors, c-Kit and many other tyrosine kinases), that result in a modification of some biological mechanisms (e.g. generally, dasatinib increases proliferation, and differentiation in AML [30]). It should be borne in mind that the functional effect resulting from the molecular action of the infused drugs, varies in practice according to several facts (for instance, the buildup of many types of mutations by some individuals). However, when we put aside all the intermediate complications that may exist in practice, we can take a shortcut that associates to each infused drug its most likely action on one or several biological functions (that are: differentiation, apoptotis, and so on), with a certain amount of confidence. Thus, we can roughly state from medical practice some major families of molecules that can be used in the case of AML or other cancers, according to their expected effect on the biological functionalities.

\begin{tabular}{|c|c|}
\hline $\begin{array}{c}\text { Fast self-renewing } \\
(\tilde{K})\end{array}$ & $\begin{array}{c}\text { Quizartinib, midostaurin } \\
\text { Dihydroorotate dehydrogenase (DHODH) inhibitors }\end{array}$ \\
\hline Apoptosis $(\tilde{\gamma})$ & Daunorubicin, cytosine arabinoside, volasertib \\
\hline Differentiation $(\tilde{\delta})$ & Dihydroorotate dehydrogenase (DHODH) inhibitors \\
\hline Cell cylce dur. $(\tilde{\tau})$ & Quizartinib, erlotinib, volasertib \\
\hline
\end{tabular}

Table 4: Here we associate the most likely (clinically established) effect of some advanced drugs/molecules on the biological features of the hematopoietic system, in the specific case of AML (without focussing neither on the molecular mechanisms behind each drug action, or on the possible mutual interactions that may exist between drugs within some combinations). The case of the sensitivity paprameter $\tilde{\alpha}$ is discussed later, in Remark 7

Remark 6. (i) One notices that some molecules in Table 4 are expected to modify more than one model parameter. For instance, the DHODH inhibitor, which is a differentiation re-activator, may decrease $\tilde{K}$ and increase $\tilde{\delta}$, since both actions seem to promote a return into normal differentiation. (ii) The volasertib (recognized as orphan drug for AML since 2014), belongs to the family of Polo-like kinase (Plk) inhibitors. It can be used in the treatment of AML to promote apoptosis and cell cycle arrest (see for instance [10]). In fact, the list of drugs given in Table 4 is not exhaustive and can be enlarged, for instance, to: histone deacetylase (HDAC) inhibitors (vorinostat and panobinostat), and the 
family of aurora kinase inhibitors (AZD115).

Now, we observe that the biological parameters considered in Table 3 imply that $2 \tilde{K} e^{-\tilde{\gamma} \tilde{\tau}}=1.078$. It follows that, theoretically, if AML is not treated, unhealthy cells will invade the bone marrow and possibly the bloodstream. In Figure 8, we illustrate the evolution of cell densities for the selected model parameters, where we observe the unbounded proliferation of unhealthy cells.

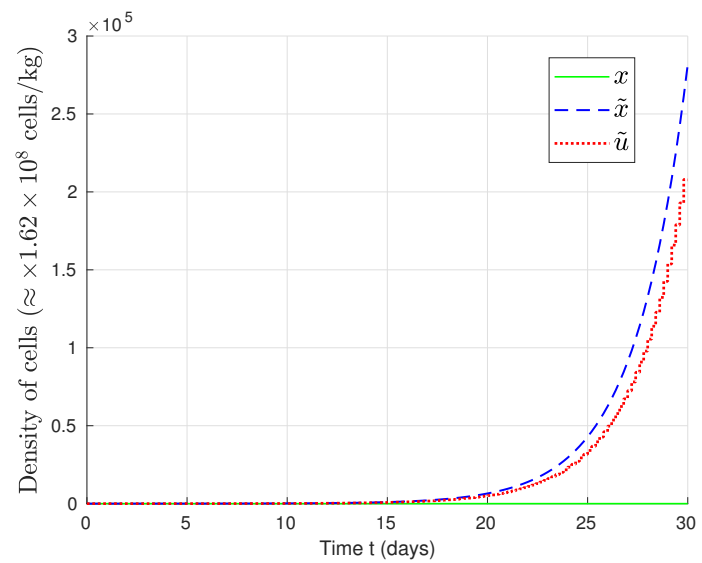

Figure 8: Trajectories of the system for the (non-treated) model parameters of Table 3

Remark 7. We expect that $\tilde{\alpha}$ is less than 1 before therapy, then it starts to increase when therapy is applied (an immunostimulating effect of cytotoxic drugs, elicited e.g. in [96, 95]), and then greater than 1 when the immune system has learnt to counter the dodges of cancer cells (such as hiding their tumor antigens or achieving inactivation of antibodies, e.g. by glycosylation), or when the reduction of the tumor burden has made immune cells proportionally more efficient in their encounters with cancer cells, or also when successful immunotherapy is used to directly target cancer cells.

Actually, the elaboration of an optimal therapeutic strategy ${ }^{16}$ is beyond the

\footnotetext{
${ }^{16}$ The optimal therapy requires the determination of the best infusion planning, that takes into account drug toxicity and other practical considerations (e.g. how the doses of each drug type are spread over the duration of the therapy). These points deserve a separated study.
} 
scope of this work. Here, we are suggesting a theoretical therapeutic strategy, that can be based on some suitable combination of drugs (listed in Table 4 or others similar ones). We assume that the resulting evolution patterns of the biological model parameters are those illustrated in Figure 9. In fact, we can distinguish between two evolution trends, nested within one another as follows:

1) The first series of infusions aims to decrease $\tilde{K}$ (fast self-renewing rate), to increase $\tilde{\tau}$ (cell-cycle duration), and to increase $\tilde{\gamma}$ (apoptosis rate). It is worth mentioning that the direction of the change in the model parameters 825 (i.e. by increasing/decreasing the model parameters values) is in line with the observed effect of the drugs listed in Table 3. This treatment phase is expected to limit the expansion of CSCs. We also assume that the first treatment phase is accompanied by a slight increase of the value of $\tilde{\alpha}$ (see Remark 7 ).

2) The second phase of the treatment aims, on the one hand, to maintain the trend given for the parameters $(\tilde{K}, \tilde{\tau}, \tilde{\gamma})$, and on the other hand, to reactivate the differentiation of unhealthy cells (using DHODH inhibitors, for instance) and to increase the sensitivity parameter $\tilde{\alpha}$ with more virulence than in the first series of infusions (e.g. using a suitable immunotherapeutic action, Remark 7).

Remark 8. It seems legitimate to wonder whether the reactivation of differentiation of CSCs is a good strategy to fight cancer. The answer is argued for instance in [28], where it is explained how CSCs can initiate and regenerate cancers, while differentiated cancer cells (called CCs [28]) will inevitably die out (see the section "Cancer stem cells and non-stem cancer cells", [28]). Thus, promoting the differentiation of CSCs into CCs appears as a sustainable way to both limit cancer progression, and avoid the escape from cancer dormancy.

Now, let us assume that an adequate combination of drugs has been fixed. We can highlight one suggestion among other possibilities, in which we propose:

(1) a shock treatment through chemotherapy that promotes apoptosis $\tilde{\gamma}$ and cell arrest $\tilde{\tau}$ (using volasertib for both objectives), and targeting $\tilde{K}$ using AC220 845 (which has also a suitable effect on cell arrest $\tilde{\tau}$ ),

(2) followed by a more differentiation-oriented treatment (using drugs based 

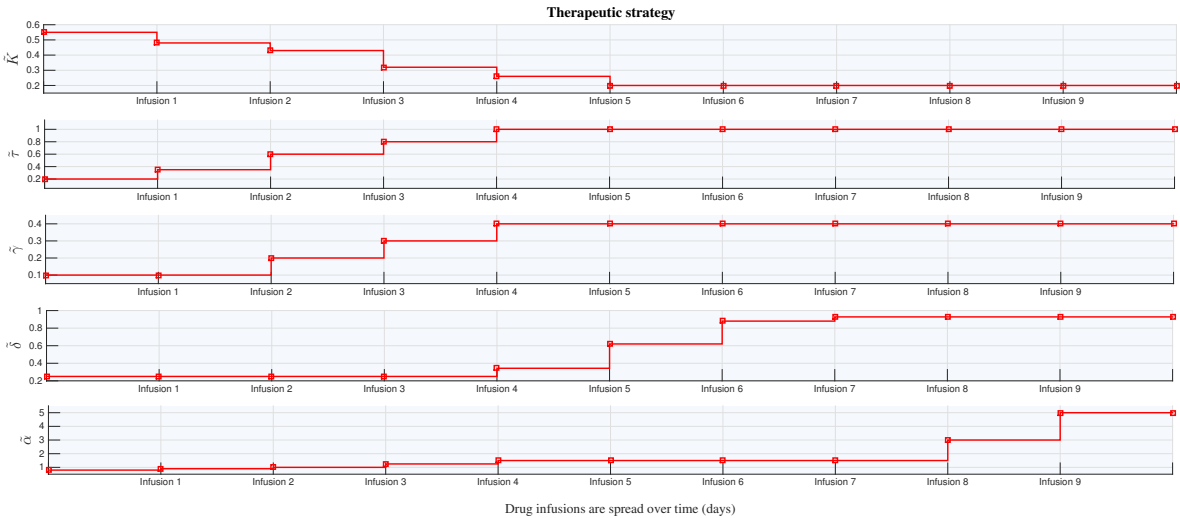

Figure 9: An illustrative therapeutic strategy that gradually modifies five model parameters, using adequates drugs: this can be achieved using a mixture of standard chemotherapy or targeted therapies, along with complementary molecules and/or immunotherapeutic actions.

on DHODH inhibitors) and mitotic/proliferation inhibition of unhealthy cells (possibly using some immunotherapy-based drugs, or vincristine, see also [79]).

We aim through the selected therapy to achieve an evolution pattern of the model parameters as close as possible to the idealistic ones given in Figure 9

Remark 9. The treatment protocol that we suggest have many similarities with classical methods in AML therapeutics [79]. We can mention in particular the $3+\boldsymbol{7}$ most famous strategy, which is also based on two main separated phases (7 days of intensive induction through cytarabine, plus 3 days of an anthracycline [79]), and then possibly followed by consolidation chemotherapy and hematopoietic cell transplant [25, 279].

Next, we apply the therapeutic strategy given in Figure 9 to our model, starting the first infusion at $t=1$ day, and considering a fixed treatment step of 1 day between successive infusions (another choice may be envisaged if needed). One notices that the model parameters after Infusion 9 are those given in Example 4 for which the decay conditions 64 of Theorem 2 are satisfied.

The evolution of the ordinary and mutated cell densities is shown in Figure 10. It is worth mentioning that in practice, the treatment of AML is spread over several separated phases. For instance, in the recent experimental work 
[85, an AML (FLT3-type) therapy based on midostaurin and chemotherapy, has been separated into two induction phases, a consolidating phase and maintenance phase (59\% of patients that have undergone the previously mentioned therapeutic protocol, then underwent bone marrow transplant, have reached the complete remission state [85]). Similarly, in our example, we assume that after Infusion 9, a consolidating and a maintenance phases continue so as to correct, adjust, strengthen, and fortify the desired dormancy state of the hematopoietic system (which is the state described by the set of parameters of Infusion 9).

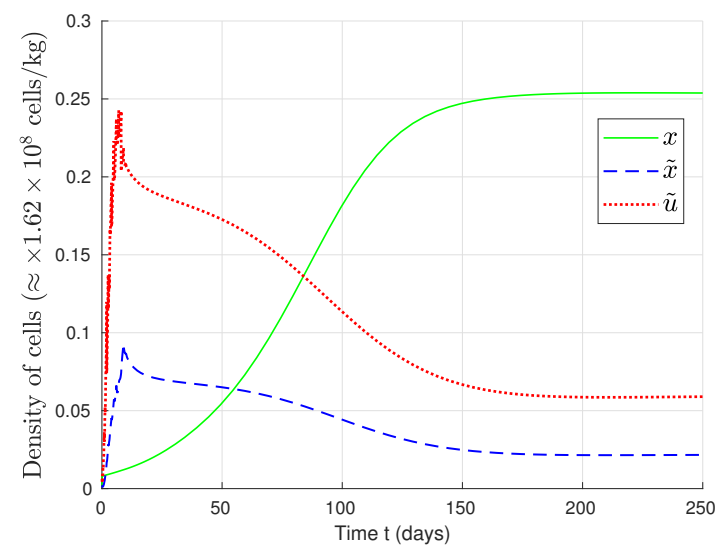

Figure 10: The evolution of the total densities of healthy and unhealthy cells (resp. $x(t)$ and $\tilde{x}(t))$ and $\tilde{u}(t)$, when we apply the theoretical therapeutic strategy illustrated in Figure 9 If we do not change the parameter values, the model behaves as in Figure 8 (i.e. CSCs overproliferate). However, in the case of treated cancer, the trajectories converge to a dormancy stable steady state, under the effect of the suggested therapy.

We conclude this work by referring to Table 1 in [79, which summarizes a number of emerging promising AML therapies, that open up other possibilities to act on cancerous hematopoietic systems. Many of these strategies can in fact be implemented and discussed within the modeling and analysis framework that we introduced in our current work. It is worth mentioning that the addition of midostaurin to chemotherapy resulted in a $22 \%$ lower risk of death among patients, in comparison to another more classical treatment (see [85]). Notice that, most of the molecules listed in [79] (and the references therein) are in early phases of development and trials, but they participate greatly, as 
well as many multidisciplinary works, to nourish this hope of moving towards systematic treatments for cancer, in general, and leukemia, in particular.

\section{References}

[1] M. Adimy, A. Chekroun, T.M. Touaoula, Age-Structured and Delay DifferentialDifference Model Of Hematopoietic Stem Cell Dynamics, Discret And Continuous Dynamical Systems Series B, Volume 20, Number 9, pp. 2765-2791, (2015).

[2] M. Adimy, F. Crauste, A. Abdllaoui, Discrete Maturity-Structured Model of Cells Differentiation with Applications to Acute Myelogenous Leukemia, J. Biological Systems, No. 3, pp. 395-424, (2008).

[3] J.A. Aguirre-Ghiso, Models, mechanisms and clinical evidence for cancer dormancy, Nature Reviews Cancer, 7(11), pp. 834-846, (2007).

[4] J.A. Aguirre-Ghiso, The problem of cancer dormancy: understanding the basic mechanisms and identifying therapeutic opportunities, Cell Cycle, Taylor \& Francis Online, Vol. 5(6), pp. 1740-1743, (2006).

[5] M. Ahsen, H. Özbay, S. I. Niculescu, Analysis of deterministic cyclic gene regulatory network models with delays, Birkhäuser, (2015).

[6] M.G. Al-Asadi, G. Brindle, M. Castellanos, S.T. May, K.I. Mills, N.H. Russell, C.H. Seedhouse, M. Pallis, A molecular signature of dormancy in CD34+CD38-acute myeloid leukaemia cells, Blood, Oncotarget, 8(67), p.111405, (2017).

[7] B. Alberts , D. Bray, K. Hopkin, A. Johnson, J. Lewis, M. Raff, K. Roberts, P. Walter, Essential Cell Biology, Fourth Edition, Garland Science, pp. 865, (2013).

[8] J. L. Avila, et al., A coupled model for healthy and cancerous cells dynamics in Acute Myeloid Leukemia, IFAC Proceedings Volumes 47.3: pp. 7529-7534, (2014).

[9] J.L. Avila, C. Bonnet, J. Clairambault, H. Özbay, S.I. Niculescu, F. Merhi, R. Tang, J.P. Marie, A new model of cell dynamics in acute myeloid leukemia involving distributed delays. IFAC TDS, pp. 55-60, (2012).

[10] J.M. Brandwein, Targeting polo-like kinase 1 in acute myeloid leukemia. Therapeutic advances in hematology, 6(2), pp. 80-87, (2015).

[11] J. Bélair, M.C. Mackey, J.M. Mahaffy, Age-structured and Two-delay Models for Erythropoiesis, Math. Biosci. 128. No. 1-2, pp. 317-346, (1995).

[12] N. Bellomo, G. Forni, Dynamics of tumor interaction with the host immune system, Mathematical and computer modelling, 20(1), pp.107-122, (1994).

[13] S. Bernard, J. Bélair, M.C. Mackey, Oscillations in cyclical neutropenia: new evidence based on mathematical modeling, Journal of theoretical biology, 223(3), pp.283-298, (2003).

[14] H. M. Blau, T. R. Brazelton, and J. M. Weimann, The Evolving Concept Review of a Stem Cell: Entity or Function?, Cell, Vol. 105, pp. 829-841, (2001).

[15] J.R. Brahmer, D.M. Pardoll, Immune checkpoint inhibitors: making immunotherapy a reality for the treatment of lung cancer, Cancer immunology research, 1(2), pp. 85-91, (2013).

[16] F.J. Burns, J.F. Tannock, On the existence of a $G_{0}$-phase in the cell cycle, Cell Tissue Kinetics, 3:321-334, (1970). 
[17] C.L. Chaffer, Brueckmann, I., Scheel, C., Kaestli, A.J., Wiggins, P.A., Rodrigues, L.O., Brooks, M., Reinhardt, F., Su, Y., Polyak, K. and Arendt, L.M., 2011. Normal and neoplastic nonstem cells can spontaneously convert to a stem-like state, Proceedings of the National Academy of Sciences, 108(19), pp.7950-7955, (2011).

[18] G. M. Crane, E. Jeffery, S.J. Morrison, Adult haematopoietic stem cell niches, Nature Reviews Immunology, (2017).

930 [19] C. Colijn, M. C. Mackey, A mathematical model of hematopoiesisI. Periodic chronic myelogenous leukemia, Journal of Theoretical Biology, 237(2), pp.117-132, (2005).

[20] F. Delhommeau, S. Dupont, V. Della Valle, C. James, S. Trannoy, A. Massé, O. Kosmider, J.P. Le-Couedic, F. Robert, A. Alberdi, Y. Lécluse, I. Plo, F.J. Dreyfus, C. Marzac, N. Casadevall, C. Lacombe, S.P. Romana, P. Dessen, J. Soulier, F. Viguié , M. Fontenay, W. Vainchenker, O.A. Bernard, Mutation in TET2 in Myeloid Cancers, New England Journal of Medicine, 360(22):2289-30, (2009).

[21] W. Djema, F. Mazenc, C. Bonnet, Stability analysis and robustness results for a nonlinear system with distributed delays describing hematopoiesis, Systems \& Control Letters, Elsevier, Vol. 102, pp. 93-101, (2017).

[22] W. Djema, F. Mazenc, C. Bonnet, Analysis of a Nonlinear Delay Differential-Difference Biological Model, IFAC-PapersOnLine, IFAC Conference on Time-Delay Systems, Vol. 49, No. 10, pp. 246-251, (2016).

[23] W. Djema, F. Mazenc, C. Bonnet, J. Clairambault, P. Hirsch, F. Delhommeau, Stability of a Delay System Coupled to a Delay Differential-Difference System Describing the Coexistence of Ordinary and Mutated Hematopoietic Stem cells, the $55^{\text {th }}$ Conference on Decision and Control, Las Vegas, USA, (2016).

[24] W. Djema, F. Mazenc, C. Bonnet, J. Clairambault, P. Hirsch, F. Delhommeau, Analysis of a Model of Dormancy in Cancer as a State of Coexistence Between Tumor and Healthy Stem Cells, Proceedings of the American Control Conference, Seattle, USA, (2017).

[25] H. Döhner, D.J. Weisdorf, C.D. Bloomfield, Acute Myeloid Leukemia, N. Engl. J. Med. 373, pp. 1136-1152, (2015).

[26] S. Ebinger, E. Z. Özdemir, C. Ziegenhain, S. Tiedt, C. C. Alves, M. Grunert, M. Dworzak, C. Lutz, V. A. Turati, T. Enver, H. P. Horny, Characterization of Rare, Dormant, and Therapy-Resistant Cells in Acute Lymphoblastic Leukemia, Cancer Cell, 30(6), pp. 849-862, (2016).

[27] R. Eftimie, J.L. Bramson, D.J. Earn, Interactions between the immune system and cancer: a brief review of non-spatial mathematical models, Bulletin of mathematical biology, 73(1), pp. 2-32, (2011).

[28] H. Enderling, Cancer Stem Cells and Tumor Dormancy, Sys. Biol. of Tumor Dormancy, Springer NY, pp. 55-71, (2013).

[29] T. Enver, M. Pera, C. Peterson, P.W. Andrews, Stem cell states, fates, and the rules of attraction, Cell Stem Cell 4, pp. 387-397, (2009).

[30] Y. Fang, L. Zhong, M. Lin, X. Zhou, H. Jing, M. Ying, M., Luo, P., Yang, B. and He, Q., $M E K / E R K$ dependent activation of STAT1 mediates dasatinib-induced differentiation of acute myeloid leukemia, PloS one, 8(6), p.e66915, (2013).

[31] M. Feuring-Buske, A.E. Frankel, R.L. Alexander, B. Gerhard, D.E. Hogge, A diphtheria toxin-interleukin 3 fusion protein is cytotoxic to primitive acute myeloid leukemia progenitors but spares normal progenitors, Cancer Research, 62(6), pp. 1730-1736, (2002). 
[32] M. Ferrarini, E. Ferrero, L. Dagna, A. Poggi, M. R. Zocchi, Human T cells: a nonredundant system in the immune-surveillance against cancer, Trends in immunology, 23(1), pp. 14-18, (2002).

[33] C. Foley, M.C. Mackey, Dynamic hematological disease: a review, J. Math. biology, 58.1-2: pp. 285-322, (2009).

[34] C. Foley, , S. Bernard, M.C. Mackey, Cost-effective G-CSF therapy strategies for cyclical neutropenia: Mathematical modelling based hypotheses, Journal of theoretical biology 238.4: pp. 754-763, (2006).

[35] J. Folkman, R. Kalluri, Cancer without disease, Nature, 427(6977), pp. 786-787, (2004).

[36] E. Fridman Stability of linear descriptor systems with delay: a Lyapunov-based approach, Journal of Mathematical Analysis and Applications, 273(1), pp. 24-44, (2002).

[37] E. Fridman, Introduction to time-delay systems: analysis and control, Birkhauser, Systems and Control: Foundations and Applications, 2014.

[38] E. Fridman, C. Bonnet, F. Mazenc, W. Djema, Stability of the cell dynamics in Acute Myeloid Leukemia, Systems \& Control Letters 88, pp. 91-100, (2016).

[39] R. A. Gatenby, A change of strategy in the war on cancer, Nature, 459(7246), pp. 508-509, (2009).

[40] C.D. Godwin, R.P. Gale, R.B. Walter, Gemtuzumab ozogamicin in acute myeloid leukemia, Leukemia, (2017).

[41] K. Gu, Y. Liu. Lyapunov-Krasovskii Functional for Uniform Stability of Coupled Differential-Functional Equations. Automatica, Vol 45, No. 3, pp. 798-804, (2009).

[42] J.K. Hale, Sufficient conditions for stability and instability of autonomous functionaldifferential equations, Journal of Differential Equations, vol. 1, pp. 452-482, (1965).

[43] E.C. Hayden, Cutting off cancer's supply lines, Nature, 458(7239), p.686-687, (2009).

[44] P. Hirsch, Y. Zhang, R. Tang, V. Joulin, H. Boutroux, E. Pronier, H. Moatti, P. Flandrin, C. Marzac, D. Bories, F. Fava, Genetic hierarchy and temporal variegation in the clonal history of acute myeloid leukaemia, Nature communications, 7, p.12475, (2016).

[45] R. Hoffman, E.J. Benz, L.E. Silberstein, H. Heslop, J. Weitz, J. Anastasi, Hematology: Basic Principles and Practice, $6^{\text {th }}$ Edition, Elsevier, Churchill Livingstone, (2012).

[46] M. Hollstein, D. Sidransky, B. Vogelstein, C.C. Harris, p53 mutations in human cancers, Science, 253(5015), pp. 49-53, (1991).

1000 [47] C. Hosing, Hematopoietic Stem Cell Mobilization with G-CSF, Methods Mol Biol. 904:37$47,(2012)$.

[48] G. Jansen, R. Gatenby, C.A. Aktipis, Control vs. eradication: Applying infectious disease treatment strategies to cancer, Proceedings of the National Academy of Sciences of United States of America, vol. 112, no. 4, pp. 937-938, (2015).

[49] C.T. Jordan, M.L. Guzman, M. Noble, Cancer stem cells, New England Journal of Medicine, 355(12), pp.1253-1261, (2006).

[50] I. Karafyllis, P. Pepe, Z.P. Jiang, Stability results for systems described by coupled retarded functional differential equations and functional difference equations, Nonlinear Anal. Theory Methods Appl. 71, 3339-3362, (2009).

1010 [51] I. Kareva, Primary and Metastatic Tumor Dormancy as a Result of Population Heterogeneity, Biology direct, 11(1), p.37., (2016).

[52] J.P. Keener, J. Sneyd, Mathematical physiology, Vol. 1, New York: Springer, (2009).

[53] V. Kolmanovskii, A. Myshkis, Introduction to the Theory and Applications of Functional Differential Equations, Kluwer Academic Publishers, Dordrecht, (1999). 
[54] E. Lainey, S. Thépot, C. Bouteloup, M. Sébert, L. Adès, M. Tailler, C. Gardin, S. Botton, A. Baruchel, P. Fenaux, G. Kroemer, S. Boehrer, Tyrosine kinase inhibitors for the treatment of acute myeloid leukemia: Delineation of anti-leukemic mechanisms of action, Biochemical Pharmacology 82, pp. 1457-1466, (2011).

[55] C.J. Langer, Emerging Immunotherapies in the Treatment of Nonsmall Cell Lung Cancer (NSCLC): The Role of Immune Checkpoint Inhibitors, American journal of clinical oncology, 38(4), pp. 422-430, (2015).

[56] A.G. McKendrick, Applications of mathematics to medical problems, Proceedings of the Edinburgh Mathematical Society 44, pp. 98-130, (1925).

[57] M.C. Mackey, Unified hypothesis of the origin of aplastic anemia and periodic hematopoiesis, Blood 51, pp. 941-956, (1978).

[58] M.C. Mackey, L. Pujo-Menjouet, J. Wu, Periodic oscillations of blood cell populations in chronic myelogenous leukemia, SIAM journal on mathematical analysis, 38(1), pp.166187, (2006).

[59] M. Malisoff, F. Mazenc, Constructions of Strict Lyapunov Functions, Serie : Communications and Control Engineering. Spinger-Verlag London Ltd, U.K., 2009.

[60] A. Marciniak-Czochra, T. Stiehl, A.D. Ho, W.Jäger, W. Wagner, Modeling of asymmetric cell division in hematopoietic stem cellsregulation of self-renewal is essential for efficient repopulation, Stem cells and development, 18(3), pp.377-386, (2009).

[61] A.N. Michel, L. Hou, D. Liu, Stability of Dynamical Systems: Continuous, Discontinuous, and Discrete Systems, Systems \& Control: Foundations \& Applications, Birkhäuser, $2^{\text {nd }}$ Ed. (2015).

[62] D. Morgan, The Cell Cycle: Principles of Control, Primers in Biology Series, Oxford University Press, pp. 297, (2006).

[63] G.N. Naumov, J. Folkman, O. Straume, Tumor dormancy due to failure of angiogenesis: role of the microenvironment. Clin Exp Metastasis, 26(1):5160, (2009).

[64] M. Nielsen, J. Thomsen, S. Primdahl, U. Dyreborg, J. Andersen, Breast cancer and atypia among young and middle-aged women: a study of 110 medicolegal autopsies, $\mathrm{Br}$. J. Cancer. 56(6):8149, (1987).

[65] H. Özbay, C. Bonnet, H. Benjelloun, J. Clairambault, Stability analysis of cell dynamics in leukemia. Math. Model Nat. Phenom., Vol. 7, No. 1, pp. 203-234, (2012).

[66] D.M. Pardoll, The blockade of immune checkpoints in cancer immunotherapy, Nature reviews. Cancer, 12(4), pp. 252, (2012).

[67] E. Passegué, C. H. Jamieson, L. E. Ailles, I. L. Weissman, Normal and leukemic hematopoiesis: Are leukemias a stem cell disorder or a reacquisition of stem cell characteristics?, Proc. Natl. Acad. Sci., Regenerative Medicine, USA 100 (Suppl 1), pp. 1184211849, (2003).

[68] P. Pepe, The Liapunov's second method for continuous time difference equations, International Journal of Robust and Nonlinear Control, 13(15), pp.1389-1405, (2003).

[69] P. Pepe, I. Karafyllis, Z.P. Jiang, On the Liapunov-Krasovskii methodology for the ISS of systems described by coupled delay differential and difference equation, Automatica, 44. pp. 2266-2273, (2008).

[70] L. Preziosi, From population dynamics to modelling the competition between tumors and immune system. Mathematical and computer modelling, 23(6), pp. 135-152, (1996). 
[71] E. Pronier, F. Delhommeau, Inhibition of TET2-mediated conversion of 5-methylcytosine to 5-hydroxymethylcytosine disturbs erythroid and granulomonocytic differentiation of human hematopoietic progenitors, Blood, 118(9), pp. 2551-2555, (2011).

[72] L. Pujo-Menjouet, Blood cell dynamics: half of a century of modelling, Mathematical Modelling of Natural Phenomena, 11(1), pp. 92-115, (2016).

[73] L. Pujo-Menjouet, S. Bernard, M.C. Mackey, Long period oscillations in a $G_{0}$ model of hematopoietic stem cells, SIAM. J. Appl. Dynam. Syst. 4(2), pp. 312-332, (2005).

[74] E. Pronier, F. Delhommeau, Role of TET2 Mutations in Myeloproliferative Neoplasms, Curr. Hematol. Malig. Rep., 7, pp. 57-64, (2012).

[75] H. Qian, Cooperativity in cellular biochemical processes: Noise-enhanced sensitivity, fluctuating enzyme, bistability with nonlinear feedback, and other mechanisms for sigmoidal responses, Annual review of biophysics, 41, pp.179-204, (2012).

[76] T. Reya, S.J. Morrison, M.F. Clarke, I.L. Weissman, Stem cells, cancer, and cancer stem cells, nature, 414(6859), pp. 105-111, (2001).

[77] A.R. Reynolds, et al., Stimulation of tumor growth and angiogenesis by low concentrations of RGD-mimetic integrin inhibitors, Nature medicine, 15(4), pp. 392-400, (2009).

[78] J.M. Rowe, B.Löwenberg, Gemtuzumab ozogamicin in acute myeloid leukemia: a remarkable saga about an active drug, Blood, 121(24), pp. 4838-4841, (2013).

[79] C. Saygin, H.E. Carraway, Emerging therapies for acute myeloid leukemia, Journal of hematology \& oncology, 10(1), p.93, (2017).

[80] R.D. Schreiber, L.J. Old, M.J. Smyth, Cancer immunoediting: integrating immunity's roles in cancer suppression and promotion, Science, 331(6024), pp. 1565-1570, (2011).

[81] J. A. Smith, L. Martin, Do cells cycle?. Proceedings of the National Academy of Sciences, 70(4), pp. 1263-1267, (1973).

[82] E. Solary, O.A. Bernard, A. Tefferi, F. Fuks, W. Vainchenker, The Ten-Eleven Translocation-2 (TET2) gene in hematopoiesis and hematopoietic diseases, Leukemia, 28 , pp. 485-496, (2014).

[83] T. Stiehl, A. Marciniak-Czochra, Characterization of stem cells using mathematical models of multistage cell lineages, Mathematical and Computer Modelling, 53(7), pp.15051517, (2011).

[84] T. Stiehl, A. Marciniak-Czochra, Mathematical modeling of leukemogenesis and cancer stem cell dynamics, Mathematical Modelling of Natural Phenomena, 7(1), pp.166-202, (2012).

[85] R.M. Stone, S.J. Mandrekar, B.L. Sanford, K. Laumann, S. Geyer, C.D. Bloomfield, C. Thiede, T.W. Prior, K. Döhner, G. Marcucci, F. Lo-Coco, Midostaurin plus Chemotherapy for Acute Myeloid Leukemia with a FLT3 Mutation, New England Journal of Medicine, (2017).

[86] D. B. Sykes, Y. S. Kfoury, F. E. Mercier, M. J. Wawer, J. M. Law, M. K. Haynes, T. A. Lewis, A. Schajnovitz, E. Jain, D. Lee, H. Meyer, Inhibition of dihydroorotate dehydrogenase overcomes differentiation blockade in acute myeloid leukemia, Cell, 167(1), pp. 171-186, (2016).

1100 [87] H.R. Thieme, Mathematics in Population Biology, Princeton Series in Theoretical and Computational Biology. Princeton University Press, (2003).

[88] B. Tuch, Stem cells - a clinical update, Australian family physician, 35(9), pp. 719, (2006). 
[89] J.W. Uhr, R.H. Scheuermann, N.E. Street, E.S. Vitetta, Cancer dormancy: opportunities for new therapeutic approaches, Nature medicine, 3(5), pp. 505-509, (1997).

[90] M.D. Vesely, et al., Natural innate and adaptive immunity to cancer, Annual review of immunology, 29, pp.235-271, (2011).

[91] G.C. Wake, The Solution and the Stability of a Nonlinear Age-Structured Population model, The ANZIAM Journal, 45(02): pp. 153-165, (2003).

[92] G. Wang, Analysis of Complex Diseases: A Mathematical Perspective, CRC Press, (2013).

[93] K.P. Wilkie, P. Hahnfeldt, Tumorimmune dynamics regulated in the microenvironment inform the transient nature of immune-induced tumor dormancy, J. Cancer Research, 73(12), pp. 353444, (2013).

[94] P.P. Zarrinkar, AC220 is a uniquely potent and selective inhibitor of FLT3 for the treatment of acute myeloid leukemia (AML), Blood, 114(14): pp. 2984-2992, (2009).

[95] L. Zitvogel, A. Tesniere, G. Kroemer, Cancer despite immunosurveillance: immunoselection and immunosubversion, Nature Reviews Immunology, 6(10), pp. 715-727, (2006).

[96] L. Zitvogel, L. Apetoh, F. Ghiringhelli, G. Kroemer, Immunological aspects of cancer chemotherapy, Nature Reviews Immunology, 8(1), pp. 58-73, (2008).

Acknowledgement: We would like to thank the A.E. and the reviewers of JTB for their valuable comments. We also thank professors P. Pepe and M. Adimy, referees of the PhD thesis of W. Djema, for the stimulating discussions we had on the topic.

\section{Appendix A. Proof of Theorem 1 (cell extinction)}

Simple calculations show that the derivative of $\mathcal{W}$, defined in (28), along the trajectories of 100 , satisfies, for almost all $t \geq 0$,

$$
\begin{aligned}
\dot{\mathcal{W}}(t)= & {\left[-\tilde{\delta}+\left(\psi_{1}-1\right) \tilde{\beta}(x(t)+\tilde{\alpha} \tilde{x}(t))\right] \tilde{x}(t) } \\
& -\left[\psi_{1}\left(e^{-\rho_{1}^{*} \tilde{\tau}}-2 \tilde{K} e^{-\tilde{\gamma} \tilde{\tau}}\right)-2(1-\tilde{K}) e^{-\tilde{\gamma} \tilde{\tau}}\right] \tilde{u}(t-\tilde{\tau}) \\
& -\left[\delta+\left(1-\psi_{2}\right) \beta(x(t)+\tilde{x}(t))\right] x(t)-\psi_{1} \rho_{1}^{*} \int_{t-\tilde{\tau}}^{t} e^{\rho_{1}^{*}(\ell-t)} \tilde{u}(\ell) d \ell \\
& -\left(\psi_{3} e^{-\rho_{2}^{*} \tau}-1\right) 2 e^{-\gamma \tau} \beta(x(t-\tau)+\tilde{x}(t-\tau)) x(t-\tau) \\
& -\psi_{2} \rho_{2}^{*} \int_{t-\tau}^{t} e^{\rho_{2}^{*}(\ell-t)} \beta(x(\ell)+\tilde{x}(\ell)) x(\ell) d \ell .
\end{aligned}
$$

Now, according to 297 , the conditions $2 \tilde{K} e^{-\tilde{\gamma} \tilde{\tau}}<1$ and $\psi^{*}>0$ are satisfied. It follows that for all $\rho_{1}^{*} \in\left(0, \frac{1}{\tilde{\tau}} \ln \left(\frac{k}{1+2(k-1) \tilde{K} e^{-\tilde{\gamma} \tilde{\tau}}}\right)\right)$, where $k>1$ is a constant 
that we will select later, we get $0<\frac{1-2 \tilde{K} e^{-\tilde{\gamma} \tilde{\tau}}}{k}<e^{-\rho_{1} \tilde{\tau}}-2 \tilde{K} e^{-\tilde{\gamma} \tilde{\tau}}<1-2 \tilde{K} e^{-\tilde{\gamma} \tilde{\tau}}$. On the other hand, using the definition of $\psi_{1}$, we can readily check that:

$$
\psi_{1}\left(1-2 \tilde{K} e^{-\tilde{\gamma} \tilde{\tau}}\right)-2(1-\tilde{K}) e^{-\tilde{\gamma} \tilde{\tau}}>0
$$

Therefore, we can notice that for all $k \in\left(1, \frac{\left(1-2 \tilde{K} e^{-\tilde{\gamma} \tilde{\tau}}\right) \psi_{1}}{2(1-\tilde{K}) e^{-\tilde{\gamma} \tilde{\tau}}}\right)$, the constant:

$$
\bar{k}=\psi_{1}\left(\frac{1-2 \tilde{K} e^{-\tilde{\gamma} \tilde{\tau}}}{k}\right)-2(1-\tilde{K}) e^{-\tilde{\gamma} \tilde{\tau}}
$$

is strictly positive. Next, since $\tilde{\beta}$ is decreasing, and using the fact that $\psi_{11}>1$, it follows that $\left(\psi_{11}-1\right) \tilde{\beta}(x(t)+\tilde{\alpha} \tilde{x}(t)) \leq\left(\psi_{11}-1\right) \tilde{\beta}(0)$. From the previous intermediate results, we conclude that for all $t \geq 0,-\tilde{\delta}+\left(\psi_{1}-1\right) \tilde{\beta}(x(t)+\tilde{\alpha} \tilde{x}(t)) \leq$ $\psi_{12} \tilde{\beta}(x(t)+\tilde{\alpha} \tilde{x}(t))$, where, $\psi_{12}<0$. Now, we assume that the third decay condition, $\delta>\left(2 e^{-\gamma \tau}-1\right) \beta(0)$, is satisfied, and we put $\psi_{3}=\frac{2 \beta(0)+(\delta+\beta(0)) e^{\gamma \tau}}{4 \beta(0)}$. Therefore, it is easy to check that, in this case, we have $\psi_{3} \in\left(1, \frac{\delta+\beta(0)}{2 \beta(0)} e^{\gamma \tau}\right)$. It follows that $\delta+\left(1-\psi_{2}\right) \beta(0)$ is positive. For later use we denote $\delta^{*}=$ $\delta+\left(1-\psi_{2}\right) \beta(0)$. Next, by selecting $\rho_{2}^{*}=\frac{1}{2 \tau} \ln \left(\frac{2 \psi_{3}}{\psi_{3}+1}\right)>0$, we deduce that $\psi_{3} e^{-\rho_{2} \tau}-1$ is positive. For later use we denote $\rho^{*}=\psi_{3} e^{-\rho_{2}^{*} \tau}-1$. We conclude that $\dot{\mathcal{W}}(t)$ satisfies, for almost all $t \geq 0$,

$$
\begin{aligned}
\dot{\mathcal{W}}(t) \leq & \psi_{12} \tilde{\beta}(x(t)+\tilde{\alpha} \tilde{x}(t)) \tilde{x}(t)-\psi_{1} \rho_{1} \int_{t-\tilde{\tau}}^{t} e^{\rho_{1}(\ell-t)} \tilde{u}(\ell) d \ell \\
& -\bar{k} \tilde{u}(t-\tilde{\tau})-2 \rho^{*} e^{-\gamma \tau} \beta(x(t-\tau)+\tilde{x}(t-\tau)) x(t-\tau) \\
& -\delta^{*} x(t)-\psi_{2} \rho_{2} \int_{t-\tau}^{t} e^{\rho_{2}(\ell-t)} \beta(x(\ell)+\tilde{x}(\ell)) x(\ell) d \ell
\end{aligned}
$$

where, $\psi_{12}<0, \bar{k}>0, \delta^{*}>0$, and, $\rho^{*}>0$. By integrating the previous inequality A.1, we deduce that the functional $\mathcal{W}$ is bounded over $[0,+\infty)$. From the definition of $\mathcal{W}$, it follows that for all $t \geq 0$, the trajectories $\tilde{x}(t)$ and $x(t)$ are bounded by, respectively, the positive constants $\tilde{x}_{s}$ and $x_{s}$. A direct 
consequence is that for almost all $t \geq 0$,

$$
\begin{aligned}
\dot{\mathcal{W}}(t) \leq & \psi_{12} \tilde{\beta}\left(x_{s}+\tilde{\alpha} \tilde{x}_{s}\right) \tilde{x}(t)-\psi_{1} \rho_{1} \int_{t-\tilde{\tau}}^{t} e^{\rho_{1}(\ell-t)} \tilde{u}(\ell) d \ell \\
& -\delta^{*} x(t)-\psi_{2} \rho_{2} \int_{t-\tau}^{t} e^{\rho_{2}(\ell-t)} \beta(x(\ell)+\tilde{x}(\ell)) x(\ell) d \ell .
\end{aligned}
$$

We conclude that for almost all $t \geq 0$, we have,

$$
\dot{\mathcal{W}}(t) \leq-\psi_{4} \mathcal{W}\left(\tilde{x}_{t}, \tilde{u}_{t}, x_{t}\right)
$$

where $\psi_{4}=\min \left\{-\psi_{12} \tilde{\beta}\left(x_{s}+\tilde{\alpha} \tilde{x}_{s}\right), \delta^{*}, \rho_{1}^{*}, \rho_{2}^{*}\right\}>0$. Now, by integrating the inequality A.2, we deduce that for all $t \geq 0$,

$$
\mathcal{W}\left(\tilde{x}_{t}, \tilde{u}_{t}, x_{t}\right) \leq e^{-\psi_{4} t} \mathcal{W}\left(\varphi_{\tilde{x}}, \varphi_{\tilde{u}}, \varphi_{x}\right)
$$

It follows from the definition of $\mathcal{W}$ that $\tilde{x}$ and $x$ converge exponentially to zero with a decay rate larger than, or equal to, $\psi_{4}$. From the second equation in 10, we note that the linearity in $\tilde{u}$ and the fact that $2 \tilde{K} e^{-\tilde{\gamma} \tilde{\tau}}<1$, imply that $\tilde{u}$ converges exponentially to the 0-equilibrium of the shifted system when $\tilde{x}$ and $x$ also converge exponentially to zero. This concludes the proof of Theorem 1 .

Appendix B. Determining $\mathfrak{s}, \tilde{\mathfrak{s}}, \mathfrak{m}$, and $\tilde{\mathfrak{m}}$, in 37 and 38

Since $R$ and $\tilde{R}$ have similar forms, we prove the desired results only for $R$. Using the expression of $\beta$ given in (1), we rewrite for all $\mathfrak{e}>0$ and $\mathfrak{z}>-\mathfrak{e}$,

$$
R(\mathfrak{z})=\beta(0)\left(\frac{1}{1+b(\mathfrak{z}+\mathfrak{e})^{n}}-\frac{1}{1+b \mathfrak{e}^{n}}\right)-\theta \mathfrak{z}
$$

Obviously, when $|\mathfrak{z}|>1$, we have

$$
\frac{\mid R(\mathfrak{z} \mid}{|\mathfrak{z}|} \leq \frac{2 \beta(0)+|\theta|}{|\mathfrak{z}|} \leq 2 \beta(0)+|\theta|
$$


To address the case where $|\mathfrak{z}| \leq 1$ for all $\mathfrak{z}>-\mathfrak{e}$ and $\mathfrak{e}>0$, we consider first the function:

$$
\rho^{\dagger}(\mathfrak{z})=\frac{1}{1+b(\mathfrak{z}+\mathfrak{e})^{n}}-\frac{1}{1+b \mathfrak{e}^{n}}=\frac{b\left[\mathfrak{e}^{n}-(\mathfrak{z}+\mathfrak{e})^{n}\right]}{q(\mathfrak{z})},
$$

where $q(\mathfrak{z})=\left[1+b(\mathfrak{z}+\mathfrak{e})^{n}\right]\left(1+b \mathfrak{e}^{n}\right)$. Using,

$$
(\mathfrak{z}+a)^{n}-a^{n}=n a^{n-1} \mathfrak{z}+n(n-1) \int_{0}^{\mathfrak{z}} \int_{a}^{a+l} m^{n-2} d m d l,
$$

we deduce that,

$$
\rho^{\dagger}(\mathfrak{z})=-n b \mathfrak{e}^{n-1} \frac{\mathfrak{z}}{q(\mathfrak{z})}+\mathfrak{C}(\mathfrak{z})
$$

where $\mathfrak{C}(\mathfrak{z})=-n b(n-1) \frac{1}{q(\mathfrak{z})} \int_{0}^{\mathfrak{z}} \int_{0}^{\ell}(m+\mathfrak{e})^{n-2} d m d \ell$. We ease the notation by considering $h=1+b \mathfrak{e}^{n}$. Then, by noticing that $\frac{1}{q(\mathfrak{z})}=\frac{1}{h}\left(\rho^{\dagger}(\mathfrak{z})+\frac{1}{h}\right)$, it follows that $\rho^{\dagger}(\mathfrak{z})=-n b \mathfrak{e}^{n-1}\left(\frac{\rho^{\dagger}(\mathfrak{z})}{h}+\frac{1}{h^{2}}\right) \mathfrak{z}+\mathfrak{C}(\mathfrak{z})$. Consequently,

$$
\rho^{\dagger}(\mathfrak{z})=-\frac{n b \mathfrak{e}^{n-1}}{h^{2}} \mathfrak{z}+\mathfrak{C}(\mathfrak{z})-\frac{n b \mathfrak{e}^{n-1}}{h} \rho^{\dagger}(\mathfrak{z}) \mathfrak{z}
$$

We recall that, by definition, $\theta=\beta^{\prime}(\mathfrak{e})=\beta(0) \frac{n b \mathfrak{e}^{n-1}}{h^{2}}$. Therefore,

$$
\rho^{\dagger}(\mathfrak{z})+\frac{\theta}{\beta(0)} \mathfrak{z}=\mathfrak{C}(\mathfrak{z})-\frac{n b \mathfrak{e}^{n-1}}{h} \rho^{\dagger}(\mathfrak{z}) \mathfrak{z}
$$

On the other hand, observe that B.1] is equivalent to $R(\mathfrak{z})=\beta(0)\left[\rho^{\dagger}(\mathfrak{z})-\frac{\theta}{\beta(0)} \mathfrak{z}\right]$. By combining the last equality with $B .5$, we get the intermediate consequence,

$$
\frac{R(\mathfrak{z})}{\beta(0)}=\mathfrak{C}(\mathfrak{z})-\frac{n b \mathfrak{e}^{n-1}}{h} \rho^{\dagger}(\mathfrak{z}) \mathfrak{z} .
$$

Now, we readily check that

$$
|\mathfrak{C}(\mathfrak{z})| \leq \frac{n b(n-1)}{q(\mathfrak{z})}(|\mathfrak{z}|+\mathfrak{e})^{n-2} \frac{\mathfrak{z}^{2}}{2}
$$


From B.3. we deduce that $\left|\rho^{\dagger}(\mathfrak{z})\right| \leq \frac{n b \mathfrak{e}^{n-1}}{q(\mathfrak{z})}|\mathfrak{z}|+|\mathfrak{C}(\mathfrak{z})|$. Using (B.7), it follows that

$$
\left|\mathfrak{z} \rho^{\dagger}(\mathfrak{z})\right| \leq \frac{n b \mathfrak{e}^{n-1}}{q(\mathfrak{z})} \mathfrak{z}^{2}+\frac{n b(n-1)}{2 q(\mathfrak{z})}(|\mathfrak{z}|+\mathfrak{e})^{n-2}|\mathfrak{z}|^{3} .
$$

Consequently, from (B.6), and using $(\mathrm{B} .7)$ and $(\mathrm{B} .8)$, we obtain the upper bound,

$$
\begin{aligned}
\frac{|R(\mathfrak{z})|}{\beta(0)} \leq & \frac{(n b)^{2}(n-1) \mathfrak{e}^{n-1}}{2 h q(\mathfrak{z})}(|\mathfrak{z}|+\mathfrak{e})^{n-2}|\mathfrak{z}|^{3} \\
& +\left[\frac{n b(n-1)}{2 q(\mathfrak{z})}(|\mathfrak{z}|+\mathfrak{e})^{n-2}+\frac{\left(n b \mathfrak{e}^{n-1}\right)^{2}}{h q(\mathfrak{z})}\right] \mathfrak{z}^{2} .
\end{aligned}
$$

On the other hand, we observe that, $\frac{1}{q(\mathfrak{z})}=\frac{1}{\left[1+b(\mathfrak{z}+\mathfrak{e})^{n}\right] h}$. Therefore, when $\mathfrak{z} \geq 0$, we have, $\frac{1}{q(\mathfrak{z})}=\frac{1}{\left[1+b(|\mathfrak{z}|+\mathfrak{e})^{n}\right] h}$, and when $\mathfrak{z} \leq 0$, then $\mathfrak{z} \in(-\mathfrak{e}, 0]$. Thus, $\frac{1}{q(\mathfrak{z})} \leq$ $\frac{1}{h} \leq \frac{1+b(2 \mathfrak{e})^{n}}{\left[1+b(|\mathfrak{z}|+\mathfrak{e})^{n}\right] h}$. Consequently, for all $\mathfrak{z}>-\mathfrak{e}$, we have,

$$
\frac{1}{q(\mathfrak{z})} \leq \frac{1+b(2 \mathfrak{e})^{n}}{\left[1+b(|\mathfrak{z}|+\mathfrak{e})^{n}\right] h}
$$

From (B.10) and (B.9), we deduce that

$$
\begin{aligned}
\frac{|R(\mathfrak{z})|}{\beta(0)} & \leq\left[\mathfrak{p}_{1} \frac{1+(|\mathfrak{z}|+\mathfrak{e})^{n-2}}{1+b(|\mathfrak{z}|+\mathfrak{e})^{n}}+\mathfrak{p}_{2} \frac{(|\mathfrak{z}|+\mathfrak{e})^{n-2}|\mathfrak{z}|}{1+b(\mid \mathfrak{z}+\mathfrak{e})^{n}}\right] \mathfrak{z}^{2} \\
& \leq\left[\mathfrak{p}_{1} \frac{1+(|\mathfrak{z}|+\mathfrak{e})^{n-2}}{1+b(|\mathfrak{z}|+\mathfrak{e})^{n}}+\mathfrak{p}_{2} \frac{(|\mathfrak{z}|+\mathfrak{e})^{n-1}}{1+b(\mid \mathfrak{z}+\mathfrak{e})^{n}}\right] \mathfrak{z}^{2},
\end{aligned}
$$

where the positive constants $\mathfrak{p}_{1}$ and $\mathfrak{p}_{2}$ are given by:

$$
\mathfrak{p}_{1}=\left[1+b(2 \mathfrak{e})^{2}\right]^{n} \max \left\{\frac{n b(n-1)}{2 h}, \frac{\left(n b \mathfrak{e}^{n-1}\right)^{2}}{h^{2}}\right\}
$$

and, $\mathfrak{p}_{2}=\frac{\left((n b)^{2}(n-1) \mathfrak{e}^{n-1}\right)\left(1+b(2 \mathfrak{e})^{n}\right)}{2 h^{2}}$. Next, observe that:

case 1: if $|\mathfrak{z}|+\mathfrak{e} \leq 1$, then $\frac{1+(|\mathfrak{z}|+\mathfrak{e})^{n-2}}{1+b(|\mathfrak{z}|+\mathfrak{e})^{n}} \leq 2$, and, $\frac{(|\mathfrak{z}|+\mathfrak{e})^{n-1}}{1+b(\mid \mathfrak{z}+\mathfrak{e})^{n}} \leq 1$.

case 2: if $|\mathfrak{z}|+\mathfrak{e}>1$, then $\frac{1+(|\mathfrak{z}|+\mathfrak{e})^{n-2}}{1+b(|\mathfrak{z}|+\mathfrak{e})^{n}} \leq \bar{b}$, and, $\frac{(|\mathfrak{z}|+\mathfrak{e})^{n-1}}{1+b(\mid \mathfrak{z}+\mathfrak{e})^{n}} \leq \bar{b}$, where, 
$\bar{b}=\max \left\{1, \frac{1}{b}\right\}$. Therefore, in both cases, we proved that:

$$
|R(\mathfrak{z})| \leq \mathfrak{m z}^{2}
$$

where, $\mathfrak{m}=\beta(0) \max \left\{\mathfrak{p}_{1} \max \left\{2, b^{-1}\right\}, \mathfrak{p}_{2} \bar{b}\right\}$.

Now, recall that $R(\mathfrak{z})=\beta(0)\left[\rho^{\dagger}(\mathfrak{z})-\frac{\theta}{\beta(0)} \mathfrak{z}\right]$. From B.11, we get,

$$
\frac{\left|\beta(0) \rho^{\dagger}(\mathfrak{z})-\theta \mathfrak{z}\right|}{|\mathfrak{z}|} \leq \mathfrak{m}|\mathfrak{z}|
$$

Therefore, we observe that if $|\mathfrak{z}| \leq 1$, the inequality $(\mathrm{B} .12)$ implies that

$$
\left|\beta(0) \rho^{\dagger}(\mathfrak{z})-\theta \mathfrak{z}\right| \leq \mathfrak{m}|\mathfrak{z}|
$$

From (B.2) and (B.13), we conclude that, for all $\mathfrak{z}>-\mathfrak{e}$ and $\mathfrak{e}>0$, we have,

$$
|R(\mathfrak{z})| \leq \mathfrak{s}|\mathfrak{z}|
$$

where $\mathfrak{s}=\max \{\mathfrak{m}, 2 \beta(0)+|\theta|\}$.

Finally, based on B.11, B.14 and similar results for $\tilde{R}$, one can easily determine constants $\mathfrak{c}_{i}$ so that $(39)$ and $(40)$ are satisfied.

\section{Appendix C. Subsequent steps in the proof of Theorem 2}

Now, we focus on the function $H$, defined after 59 . We recall that there exist $\mathfrak{c}_{i}>0, i=1, \ldots, 6$ such that (39) and 40) are satisfied. In addition, from the expression of $V^{\dagger}$, defined in $(53)$, we notice that since $\lambda_{1}=2$, we get,

$$
\begin{aligned}
& V^{\dagger}\left(X_{t}, \tilde{X}_{t}, \tilde{U}_{t}\right) \geq \frac{\mathfrak{c}_{1}}{\max \left\{\mathfrak{c}_{1}, \mathfrak{c}_{2}\right\}} Q(X(t))+\frac{\mathfrak{c}_{2}}{\max \left\{\mathfrak{c}_{1}, \mathfrak{c}_{2}\right\}} Q(\tilde{X}(t), \\
& |\tilde{X}(t)| \leq \sqrt{V^{\dagger}\left(X_{t}, \tilde{X}_{t}, \tilde{U}_{t}\right)}, \quad \text { and }, \quad|X(t)| \leq \sqrt{2 V^{\dagger}\left(X_{t}, \tilde{X}_{t}, \tilde{U}_{t}\right)} .
\end{aligned}
$$


By combining the previous inequalities, we get the following upper bound:

$$
\begin{aligned}
\left|H\left(X_{t}, \tilde{X}_{t}\right)\right| \leq v V^{\dagger^{2}}\left(X_{t}, \tilde{X}_{t}, \tilde{U}_{t}\right)+\mathfrak{c}_{5} \sqrt{2 V^{\dagger}\left(X_{t}, \tilde{X}_{t}, \tilde{U}_{t}\right)} Q(X(t-\tau)) \\
+\left[\lambda_{4} \mathfrak{c}_{1}\left(\mathfrak{a}_{4}+\mathfrak{a}_{2}\right)+\mathfrak{c}_{3}\right] \sqrt{2 V^{\dagger}\left(X_{t}, \tilde{X}_{t}, \tilde{U}_{t}\right)} Q(X(t)) \\
+\left[\lambda_{4} \mathfrak{c}_{2}\left(\mathfrak{a}_{4}+\mathfrak{a}_{2}\right)+\mathfrak{c}_{4}\right] \sqrt{2 V^{\dagger}\left(X_{t}, \tilde{X}_{t}, \tilde{U}_{t}\right)} Q(\tilde{X}(t)) \\
+\mathfrak{c}_{6} \sqrt{2 V^{\dagger}\left(X_{t}, \tilde{X}_{t}, \tilde{U}_{t}\right)} Q(\tilde{X}(t-\tau)),
\end{aligned}
$$

where, $v=\frac{\left(\mathfrak{d}_{1} \lambda_{4}+2\left(\mathfrak{a}_{5} \lambda_{4}\right)^{2}\right) \max \left\{\mathfrak{c}_{1}, \mathfrak{c}_{2}\right\}^{2}}{2 \mathfrak{d}_{1}}$. A direct consequence is that the time derivative of $V^{\dagger}$ satisfies for almost all $t \geq 0$,

$$
\begin{aligned}
\dot{V}^{\dagger}(t) \leq & -2 \overline{\mathfrak{d}} V^{\dagger}\left(X_{t}, \tilde{X}_{t}, \tilde{U}_{t}\right)-\frac{\mathfrak{d}_{1}}{2} Q(\tilde{U}(t-\tilde{\tau})) \\
& -\left[\overline{\mathfrak{d}}-v V^{\dagger}\left(X_{t}, \tilde{X}_{t}, \tilde{U}_{t}\right)\right] V^{\dagger}\left(X_{t}, \tilde{X}_{t}, \tilde{U}_{t}\right) \\
& -\left[\frac{\mathfrak{d}_{4}}{2}-\left(\lambda_{4} \mathfrak{c}_{2}\left(\mathfrak{a}_{4}+\mathfrak{a}_{2}\right)+\mathfrak{c}_{4}\right) \sqrt{2 V^{\dagger}\left(X_{t}, \tilde{X}_{t}, \tilde{U}_{t}\right)}\right] Q(\tilde{X}(t)) \\
& -\left[\mathfrak{d}_{2}-\mathfrak{c}_{5} \sqrt{2 V^{\dagger}\left(X_{t}, \tilde{X}_{t}, \tilde{U}_{t}\right)}\right] Q(X(t-\tau)) \\
& -\left[\frac{\mathfrak{d}_{5}}{2}-\left(\lambda_{4} \mathfrak{c}_{1}\left(\mathfrak{a}_{4}+\mathfrak{a}_{2}\right)+\mathfrak{c}_{3}\right) \sqrt{2 V^{\dagger}\left(X_{t}, \tilde{X}_{t}, \tilde{U}_{t}\right)}\right] Q(X(t)) \\
& -\left[\mathfrak{d}_{3}-\mathfrak{c}_{6} \sqrt{2 V^{\dagger}\left(X_{t}, \tilde{X}_{t}, \tilde{U}_{t}\right)}\right] Q(\tilde{X}(t-\tau)) .
\end{aligned}
$$

Consequently, for all initial conditions belonging to the set

$$
\mathcal{B}=\left\{\left(\varphi_{X}, \varphi_{\tilde{X}}, \varphi_{\tilde{U}}\right) \in \mathcal{C}_{\tau} \times \tilde{\mathcal{C}}_{\tau} \times \tilde{\mathcal{C}}_{\tilde{\tau}} \mid V^{\dagger}\left(\varphi_{X}, \varphi_{\tilde{X}}, \varphi_{\tilde{U}}\right)<\bar{V}^{\dagger}\right\}
$$

where, with an abuse of notation, we consider the spaces of continuous functions: $\mathcal{C}_{\tau}=\mathcal{C}\left([-\tau, 0],\left(-x_{e},+\infty\right)\right), \tilde{\mathcal{C}}_{\tau}=\mathcal{C}\left([-\tau, 0],\left(-\tilde{x}_{e},+\infty\right)\right)$, and, $\tilde{\mathcal{C}}_{\tilde{\tau}}=$ $\mathcal{C}\left([-\tilde{\tau}, 0],\left(-\tilde{u}_{e},+\infty\right)\right)$, as well as the upper bound: $\bar{V}^{\dagger}=\min \left\{\frac{\overline{\mathrm{g}}}{v}, u_{1}^{2}, u_{2}^{2}, u_{3}^{2}, u_{4}^{2}\right\}$, where, $u_{1}=\frac{\mathfrak{o}_{4}}{8\left(\lambda_{4} \mathfrak{c}_{2}\left(\mathfrak{a}_{4}+\mathfrak{a}_{2}\right)+\mathfrak{c}_{4}\right)}, u_{2}=\frac{\mathfrak{o}_{5}}{8\left(\lambda_{4} \mathfrak{c}_{1}\left(\mathfrak{a}_{4}+\mathfrak{a}_{2}\right)+\mathfrak{c}_{3}\right)}, u_{3}=\frac{\mathfrak{o}_{4}}{4 \mathfrak{c}_{5}}$, and, $u_{4}=\frac{\mathfrak{o}_{3}}{4 \mathfrak{c}_{6}}$,

we finally find that the derivative of the functional $V^{\dagger}$ satisfies:

$$
\dot{V}^{\dagger}(t) \leq-2 \overline{\mathfrak{d}} V^{\dagger}\left(X_{t}, \tilde{X}_{t}, \tilde{U}_{t}\right) \text {, where } \overline{\mathfrak{d}}>0 \text {, for almost all } t \geq 0 .
$$

